Supporting Information for:

\title{
Inhibitory Effects of the Guanine Moiety on Suzuki Couplings of Unprotected Halonucleosides in Aqueous Media
}

\author{
Elizabeth C. Western and Kevin H. Shaughnessy* \\ Department of Chemistry and Center for Green Manufacturing, The University of \\ Alabama, Box 870336, Tuscaloosa, AL 35487-0336
}

Table of Contents

Experimental Details.......................................................

References................................................................

NMR Spectra for 8-bromo-1-methyl-2 Gdeoxyguanosine $\ldots \ldots \ldots \ldots \ldots \ldots \ldots \ldots \ldots \ldots . .5$

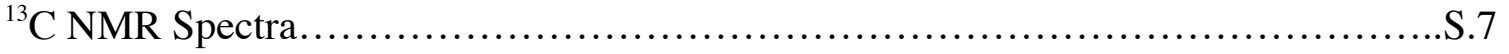

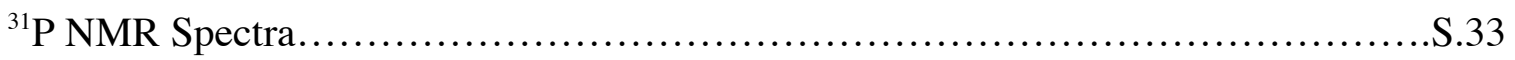




\section{Experimental details}

General. Palladium acetate and TPPTS were obtained from Strem and used as received. $\mathrm{PhB}(\mathrm{OH})_{2}$ was obtained from Aldrich and used without further purification. 8-bromo-2 $\mathrm{G}$ deoxyadenosine, ${ }^{1} 8$-bromo-2马deoxyguanosine $(8 \mathrm{BrdG}),{ }^{2} 1$-methyl-2घdeoxyguanosine $(1 \mathrm{MedG})^{3}$ and TXPTS $^{4}$ were prepared according to literature methods. 8-bromo-1methyl-2-deoxyguanosine ( $8 \mathrm{Br}-1 \mathrm{MedG})$ was prepared by modifying the procedure for bromination of dG. ${ }^{2,5}$ Water and acetonitrile were combined and sparged for 15 minutes with nitrogen prior to use.

8-bromo-1-methyl-2Gdeoxyguanosine. ${ }^{2.5} 1 \mathrm{MedG}(283.3 \mathrm{mg}, 0.95 \mathrm{mmol})$ and recently recrystallized NBS $(270.1 \mathrm{mg}, 1.5 \mathrm{mmol})$ were collected and the dry white mixture was stirred before adding $\mathrm{H}_{2} \mathrm{O}(50 \mathrm{~mL})$ to give a bright yellow mixture. This mixture was stirred for no more than 30 minutes at room temperature and then filtered over a glass frit under reduced pressure. The filter cake was washed with $\mathrm{H}_{2} \mathrm{O}$ and dried to give 254.5 $\mathrm{mg}, 71 \%$ yield. No further purification was needed. ${ }^{1} \mathrm{H}$ NMR (360 MHz, DMSO-d $\left.{ }_{6}\right)$ : $7.07(\mathrm{~s}, 2 \mathrm{H}), 6.13(\mathrm{t}, J=6.9 \mathrm{~Hz}, 1 \mathrm{H}), 5.21(\mathrm{~d}, J=4.2 \mathrm{~Hz}, 1 \mathrm{H}), 4.77(\mathrm{t}, J=5.9 \mathrm{~Hz}, 1 \mathrm{H}), 4.39$ (br s, 1H), 3.77 (br s, 1H), $3.61(\mathrm{~m}, 1 \mathrm{H}), 3.49(\mathrm{~m}, 1 \mathrm{H}), 3.28(\mathrm{~s}, 3 \mathrm{H}), 3.20(\mathrm{~m}, 1 \mathrm{H}), 2.08$ (m, 1H). ${ }^{13} \mathrm{C}$ NMR $(125.8 \mathrm{MHz}$, DMSO-d 6 ): $\mathrm{D} 155.1,153.7,150.1,120.6,116.4,87.7$, 84.8, 70.9, 61.9, 36.1, 28.0 .

General procedure for HPLC experiments. All reagents were assembled in a nitrogen drybox in vials closed with screw-cap septa. Once the vials were removed from the drybox, solvent ( $1 \mathrm{~mL} \mathrm{2:1} \mathrm{water-acetonitrile)} \mathrm{was} \mathrm{added} \mathrm{via} \mathrm{syringe} \mathrm{and} \mathrm{reactions} \mathrm{were}$ allowed to stir in an oil bath. Aliquots (ca. $20 \square \mathrm{L}$ ) were removed periodically via syringe, diluted with methanol (ca. $2 \mathrm{~mL}$ ) and placed in a container packed with ice. Reaction progress was monitored by RP-HPLC (C-18 column, eluted with gradient ranging from $10 \% \mathrm{MeOH}$ in $\mathrm{H}_{2} \mathrm{O}$ to $80 \% \mathrm{MeOH}$ in $\mathrm{H}_{2} \mathrm{O}$, with detection at $263 \mathrm{~nm}$ ). In general, under these elution conditions the retention times were as follows: $8 \mathrm{BrdA}(15.2$ min), 8PhdA (17.3 min), 8BrdG (13.4 min), 8PhdG (16.3 min), 8Br-1MedG (14.4 min) and $8 \mathrm{Ph}-1 \mathrm{MedG}(16.9 \mathrm{~min})$.

HPLC competition experiments. Reactions were set up and monitored according to the general procedure. $\mathrm{Pd}(\mathrm{OAc})_{2}(2.3 \mathrm{mg}, 0.01 \mathrm{mmol})$, TPPTS $(15.4 \mathrm{mg}, 0.027 \mathrm{mmol})$, $\mathrm{Na}_{2} \mathrm{CO}_{3}(24.2 \mathrm{mg}, 0.23 \mathrm{mmol}), 8 \mathrm{BrdA}(36.8 \mathrm{mg}, 0.11 \mathrm{mmol}), 8 \mathrm{BrdG}(38.7 \mathrm{mg}, 0.11$ $\mathrm{mmol})$ or $8 \mathrm{Br}-1 \mathrm{MedG}(38.5 \mathrm{mg}, 0.10 \mathrm{mmol})$, and $\mathrm{PhB}(\mathrm{OH})_{2}(7.3 \mathrm{mg}, 0.06 \mathrm{mmol})$ were collected in a nitrogen drybox in vials closed with screw-cap septa. Once the vials were removed from the drybox, solvent (2:1 water-acetonitrile) was added via syringe and the reaction vials were placed in a $50{ }^{\circ} \mathrm{C}$ oil bath.

HPLC additive experiments. Reactions were set up and monitored according to the general procedure. In general, $\mathrm{Pd}(\mathrm{OAc})_{2}(2.2 \mathrm{mg}, 0.01 \mathrm{mmol})$, TPPTS $(14.2 \mathrm{mg}, 0.025$ mmol), $\mathrm{Na}_{2} \mathrm{CO}_{3}(21-32 \mathrm{mg}, 0.2-0.3 \mathrm{mmol}), 8 \mathrm{BrdA}(33 \mathrm{mg}, 0.1 \mathrm{mmol})$ and $\mathrm{PhB}(\mathrm{OH})_{2}$ $(18.2 \mathrm{mg}, 0.15 \mathrm{mmol})$ along with the additive (dA, dG or $1 \mathrm{MedG})$ in varying amounts were collected in a nitrogen drybox in vials closed with screw-cap septa. Once the vials 
were removed from the drybox, solvent (2:1 water-acetonitrile) was added via syringe and the reaction vials were placed in a $50{ }^{\circ} \mathrm{C}$ oil bath.

General procedure for GC experiments. $\mathrm{Pd}(\mathrm{OAc})_{2}(2.2 \mathrm{mg}, 0.01 \mathrm{mmol})$, TPPTS (14.2 $\mathrm{mg}, 0.025 \mathrm{mmol})$ or TXPTS (16.3 mg, $0.025 \mathrm{mmol}), \mathrm{Na}_{2} \mathrm{CO}_{3}(27.5$ - $28.7 \mathrm{mg}, 0.26-$ $0.27 \mathrm{mmol})$ and $\mathrm{PhB}(\mathrm{OH})_{2}(18.9 \mathrm{mg}, 0.156 \mathrm{mmol})$ were assembled in a nitrogen drybox in vials closed with screw-cap septa. Once the vials were removed from the drybox, 4bromotoluene (12.5 $\square \mathrm{L}, 0.1 \mathrm{mmol})$ and solvent ( $1 \mathrm{~mL} \mathrm{2:1} \mathrm{water-acetonitrile)} \mathrm{were} \mathrm{added}$ via syringe and reactions were allowed to stir in a $50{ }^{\circ} \mathrm{C}$ oil bath. Aliquots (ca. $20 \square \mathrm{L}$ ) were removed periodically via syringe. These aliquots were diluted with ethyl acetate (ca. $2 \mathrm{~mL}$ ) and the internal standard mesitylene (10 $\square \mathrm{L}$ ) added. Samples were then analyzed by GC (Alltech EC ${ }^{\mathrm{TM}}$-WAX capillary column, FID detector). Product yields were calculated based on calibrations obtained using authentic compounds and mesitylene as internal standard.

General procedure for NMR experiments. $\mathrm{Pd}(\mathrm{OAc})_{2}, \mathrm{Na}_{2} \mathrm{CO}_{3}, \mathrm{TXPTS}, \mathrm{TPPTS}, \mathrm{dG}$, $1 \mathrm{MedG}, \mathrm{dA}$ and $\mathrm{PhB}(\mathrm{OH})_{2}$ were collected in varying amounts depending on the system being studied in a nitrogen drybox in vials closed with screw-cap septa. The vials were brought out of the drybox and solvent added via syringe. A portion of this solution (2.5 $\mathrm{mL}$ for the $10 \mathrm{~mm}$ tubes or $0.5 \mathrm{~mL}$ for the $5 \mathrm{~mm}$ tubes) was transferred via syringe to a septum-sealed NMR tube under nitrogen. In most cases, samples were analyzed immediately after solvent was added and then analyzed at intervals as indicated after that. All spectra were collected using Bruker $360 \mathrm{MHz}$ or $500 \mathrm{MHz}$ NMR instruments. ${ }^{13} \mathrm{C}$ NMR spectra were references to the acetonitrile $\left(\mathrm{CD}_{3} \mathrm{CN}\right)$ solvent resonance. ${ }^{31} \mathrm{P}$ NMR spectra were externally referenced to $85 \% \mathrm{H}_{3} \mathrm{PO}_{4}(0 \mathrm{ppm})$ or internally to the phosphine oxide resonance $($ TPPTS $=32.3 \mathrm{ppm}$, TXPTS=37.6 ppm) 


\section{References}

(1) Wood, M. L.; Esteve, A.; Morningstar, M. L.; Kuziemko, G. M.; Essigmann, J. M. Nucl. Acids. Res. 1992, 20, 6023-6032.

(2) Gannett, P. M.; Sura, T. P. Synth. Commun. 1993, 23, 1611-1615.

(3) McBride, L. J.; Kierzek, R.; Beaucage, S. L.; Caruthers, M. H. J. Am. Chem. Soc. 1986, 108, 2040-2048.

(4) Gulyás, H.; Szöllösy, Á.; Hanson, B. E.; Bakos, J. Tetrahedron Lett. 2002, 43, 2543-2546.

(5) Personal communication with Jonathan Daft and Professor Peter Gannett, West Virginia University, School of Pharmacy 
${ }^{1} \mathrm{H}$ NMR spectrum of 8-bromo-1-methyl-2'-deoxyguanosine (DMSO-d ${ }_{6}$ )

2.0

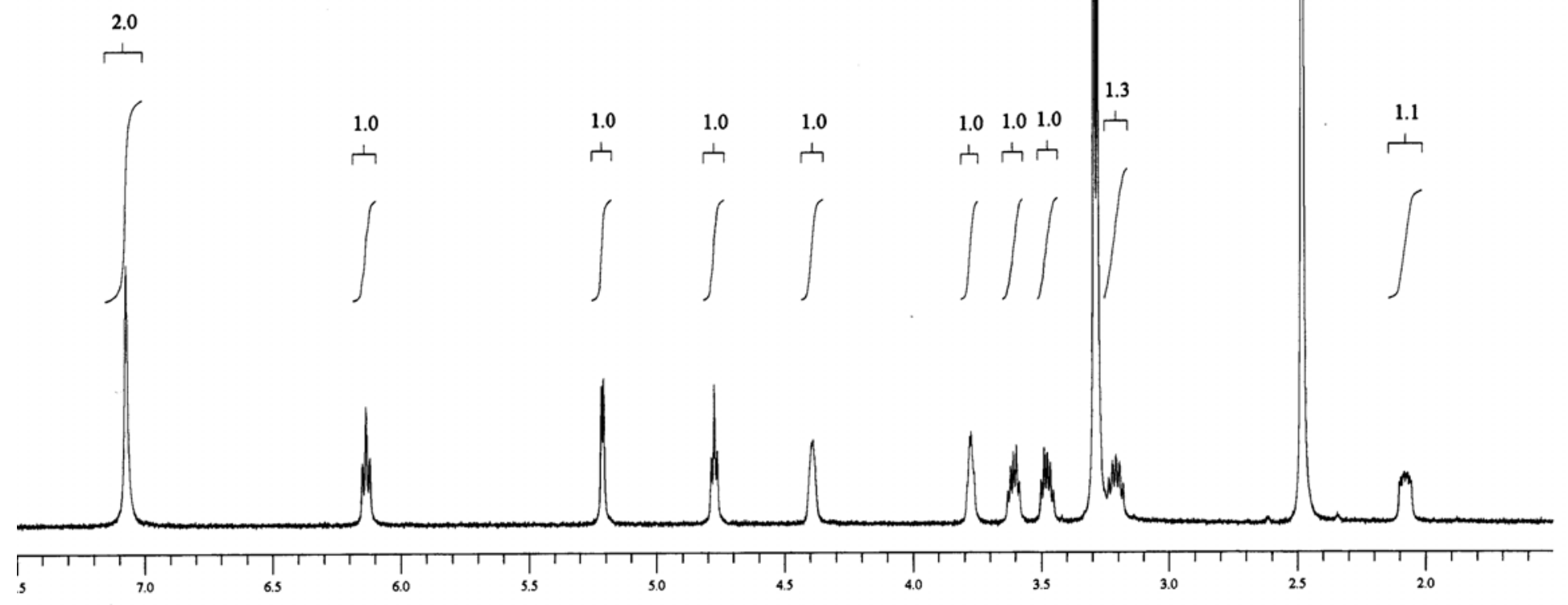


${ }^{13} \mathrm{C}$ NMR spectrum of 8-bromo-1-methyl-2'-deoxyguanosine (DMSO- $\mathrm{d}_{6}$ )

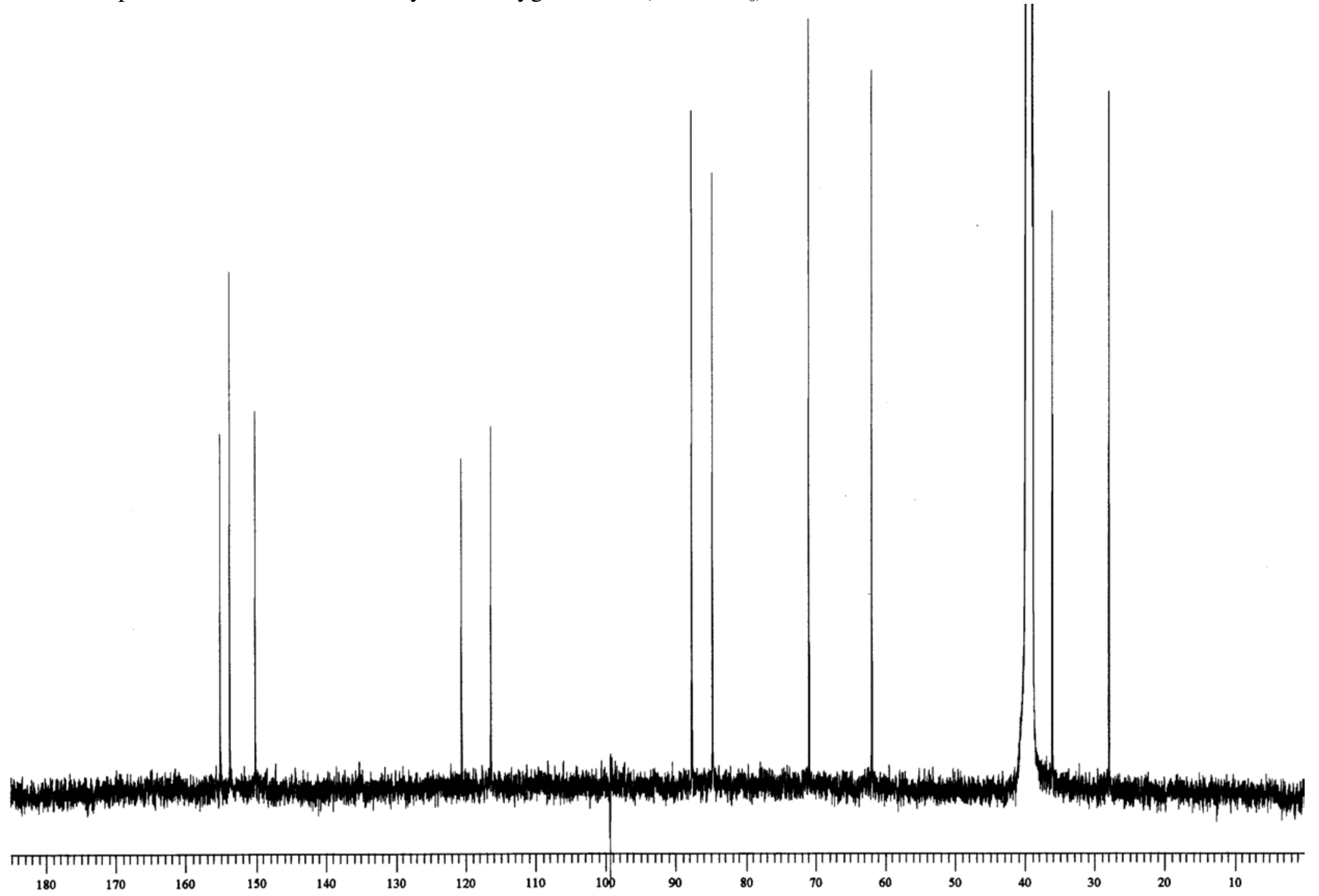




\begin{tabular}{|l|l|}
\hline 2'-deoxyguanosine $(\mathrm{dG})$ & $0.1 \mathrm{M}$ \\
\hline $\mathrm{Na}_{2} \mathrm{CO}_{3}$ & $3.2 \mathrm{eq}$ \\
\hline $2: 1 \mathrm{D}_{2} \mathrm{O}-\mathrm{CD}_{3} \mathrm{CN}$ \\
\hline $90.6 \mathrm{MHz}$ \\
\hline $5 \mathrm{~mm} \mathrm{NMR}$ tube \\
\hline
\end{tabular}

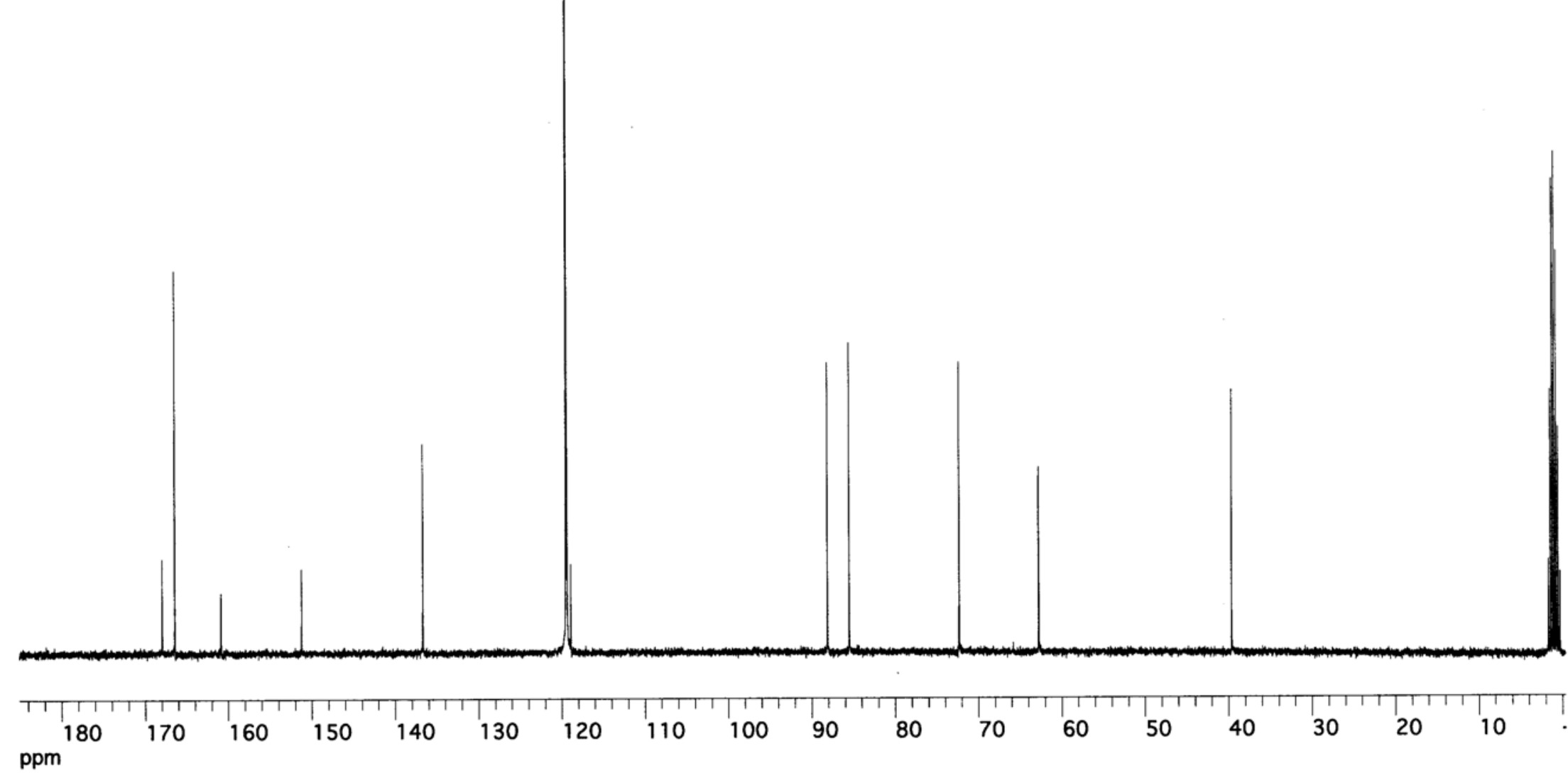




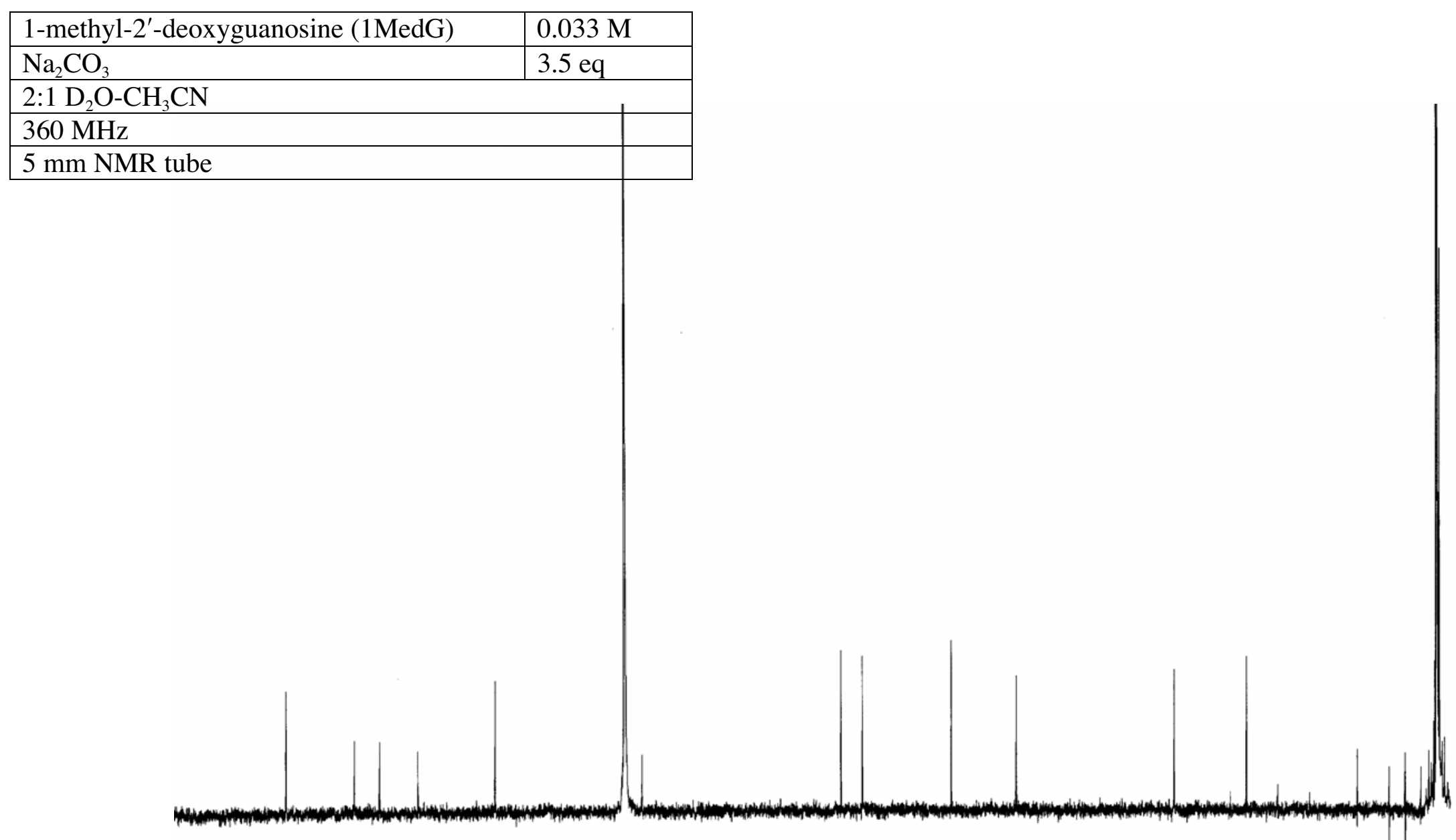

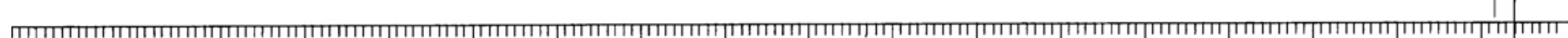
180.000
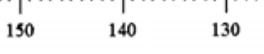

120

$110 \quad 100$

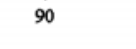




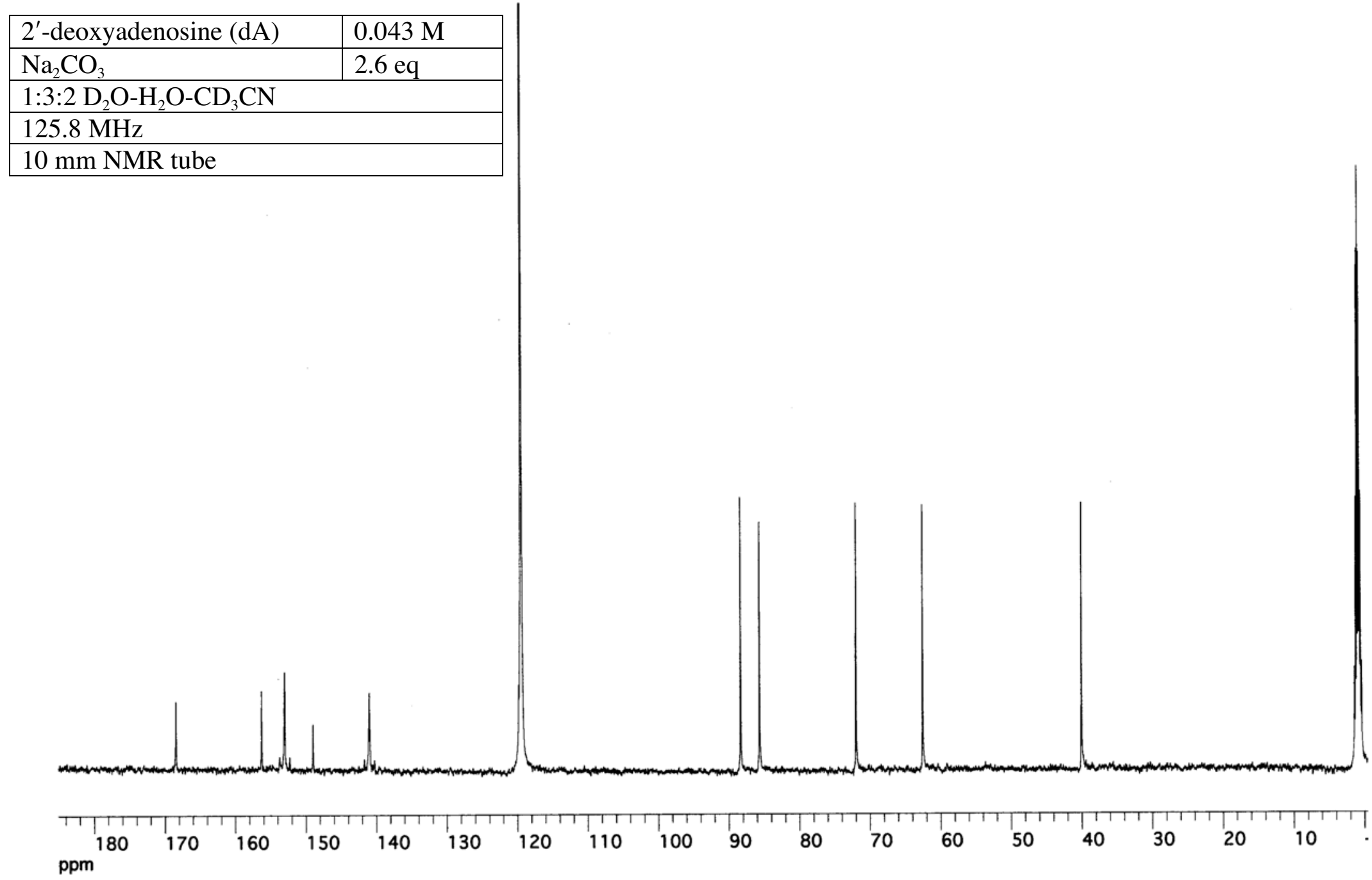




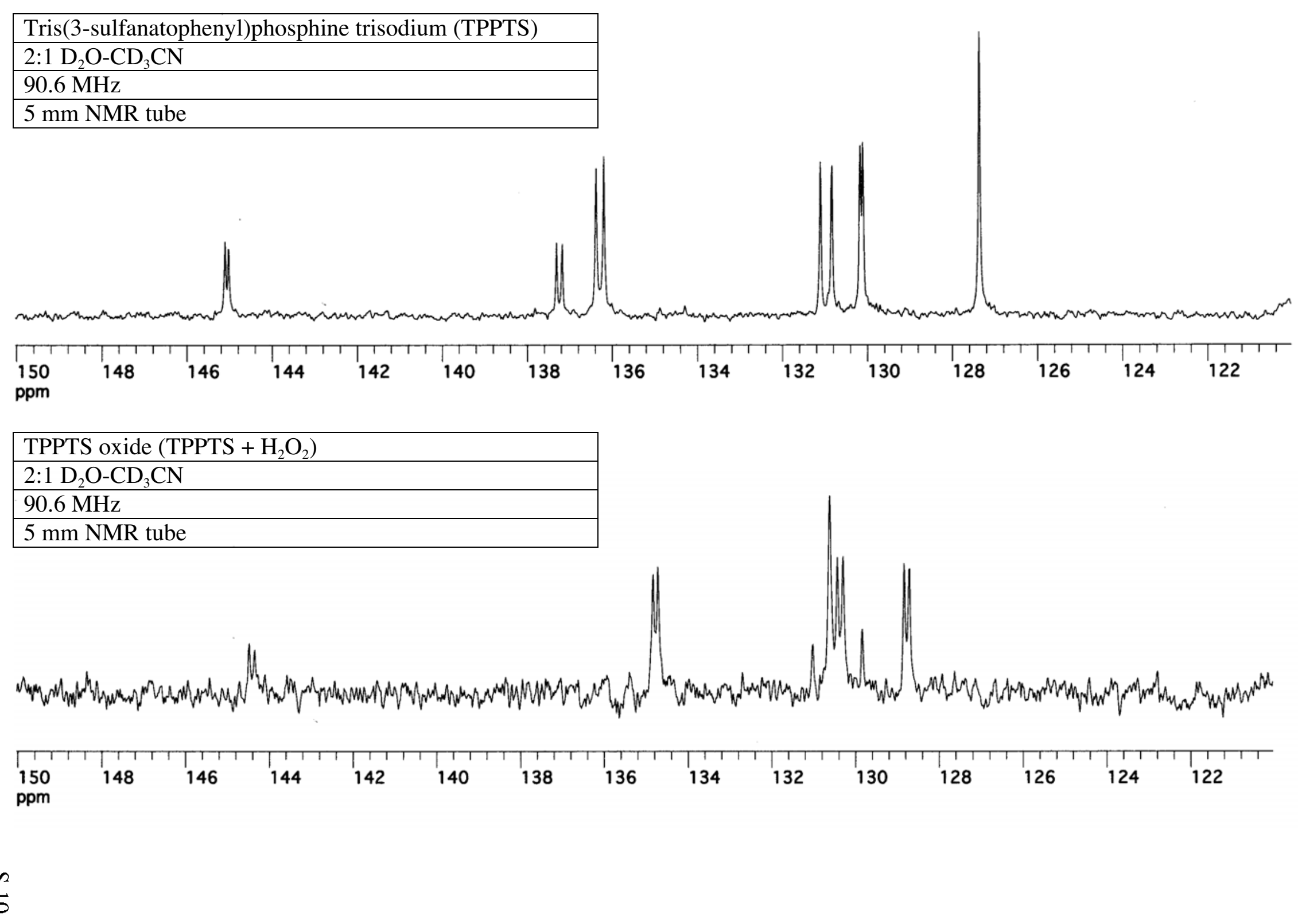




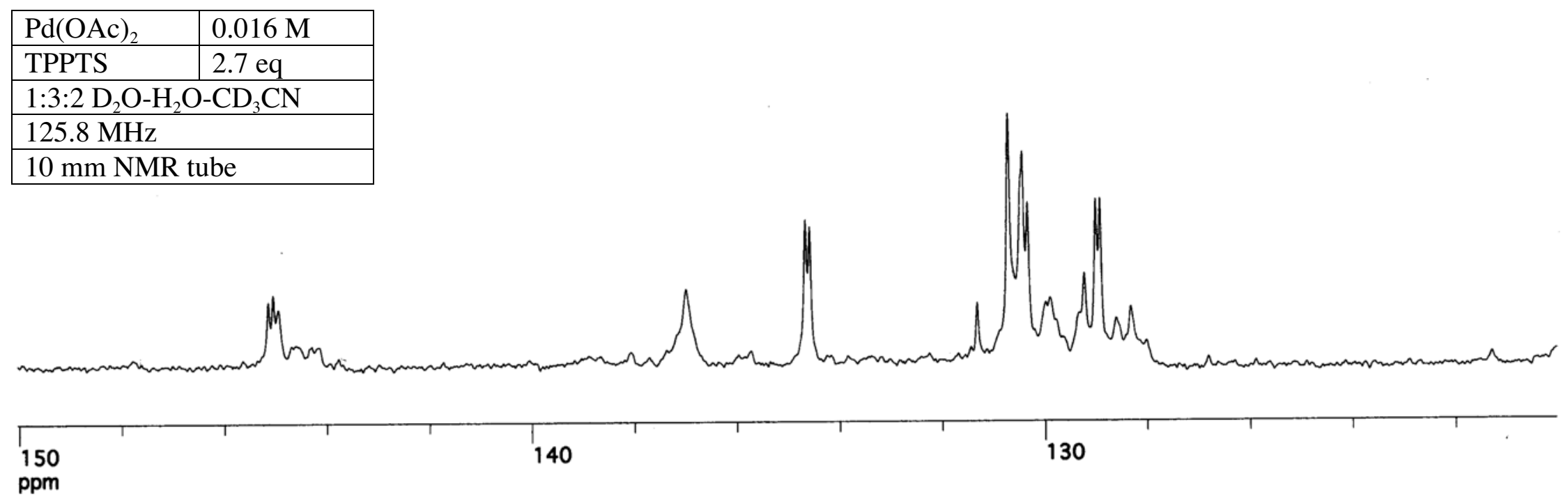

\begin{tabular}{|l|l|}
\hline $\mathrm{Pd}(\mathrm{OAc})_{2}$ & $0.017 \mathrm{M}$ \\
\hline TPPTS & $2.5 \mathrm{eq}$ \\
\hline $\mathrm{Na}_{2} \mathrm{CO}_{3}$ & $6.4 \mathrm{eq}$ \\
\hline $1: 3: 2 \mathrm{D}_{2} \mathrm{O}-\mathrm{H}_{2} \mathrm{O}-\mathrm{CD}_{3} \mathrm{CN}$ \\
\hline $125.8 \mathrm{MHz}$ \\
\hline $10 \mathrm{~mm} \mathrm{NMR}$ tube \\
\hline
\end{tabular}

(n)

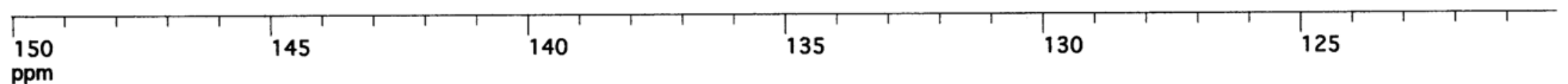




\begin{tabular}{|l|l|}
\hline $\mathrm{Pd}(\mathrm{OAc})_{2}$ & $0.02 \mathrm{M}$ \\
\hline $\mathrm{Na}_{2} \mathrm{CO}_{3}$ & $3.1 \mathrm{eq}$ \\
\hline TPPTS & $2.5 \mathrm{eq}$ \\
\hline $\mathrm{dG}$ & $1.1 \mathrm{eq}$ \\
\hline $2: 1 \mathrm{H}_{2} \mathrm{O}-\mathrm{CH}_{3} \mathrm{CN}$ & \\
\hline $125.8 \mathrm{MHz}$ & \\
\hline $10 \mathrm{~mm} \mathrm{NMR}$ tube \\
\hline
\end{tabular}
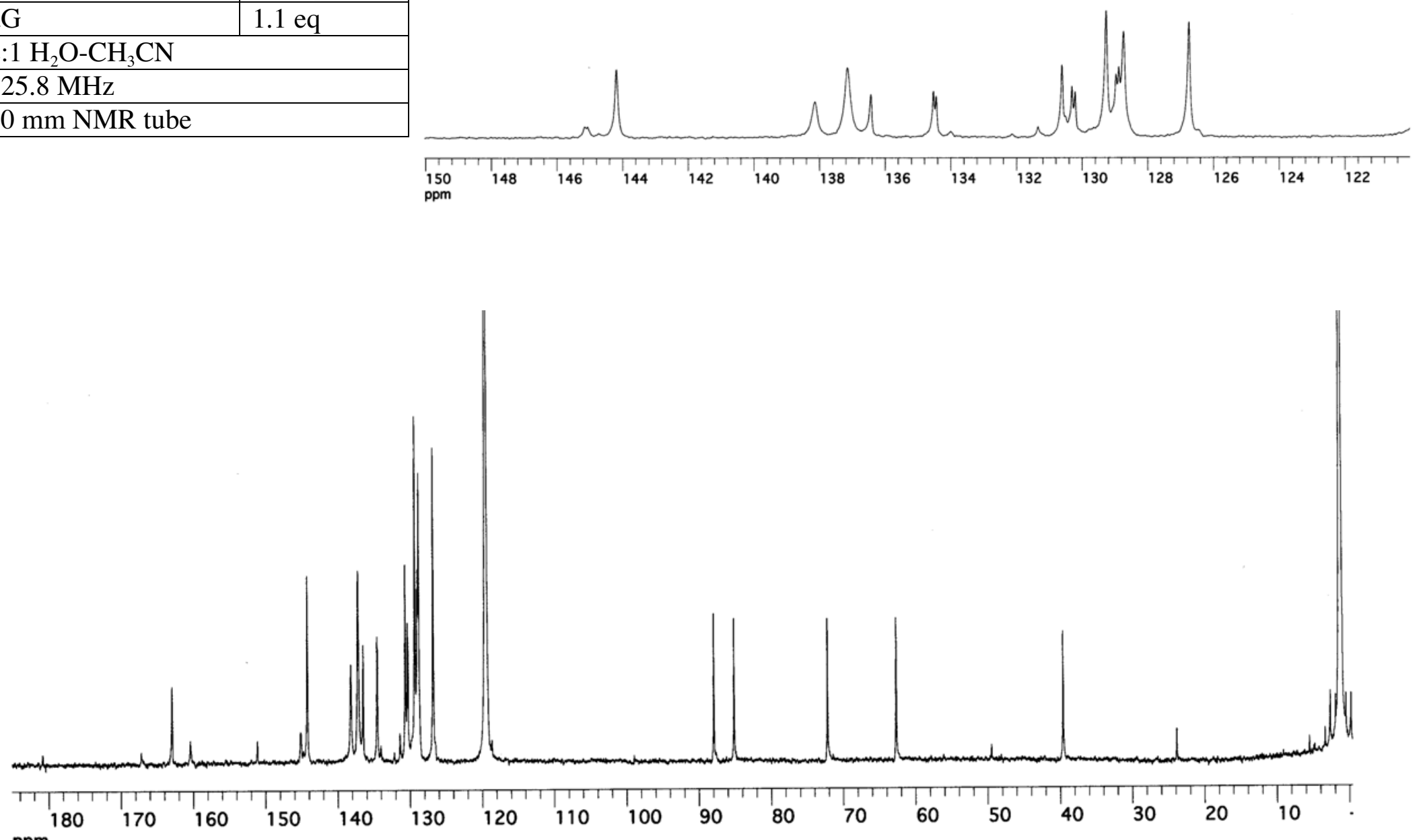

ppm 


\begin{tabular}{|l|l|}
\hline $\mathrm{Pd}(\mathrm{OAc})_{2}$ & $0.017 \mathrm{M}$ \\
\hline $\mathrm{Na}_{2} \mathrm{CO}_{3}$ & $6.0 \mathrm{eq}$ \\
\hline TPPTS & $2.5 \mathrm{eq}$ \\
\hline $\mathrm{dG}$ & $2.0 \mathrm{eq}$ \\
\hline $1: 3: 2 \mathrm{D}_{2} \mathrm{O}-\mathrm{H}_{2} \mathrm{O}-\mathrm{CH}_{3} \mathrm{CN}$ \\
\hline $125.8 \mathrm{MHz}$ \\
\hline $10 \mathrm{~mm} \mathrm{NMR} \mathrm{tube}$ \\
\hline
\end{tabular}
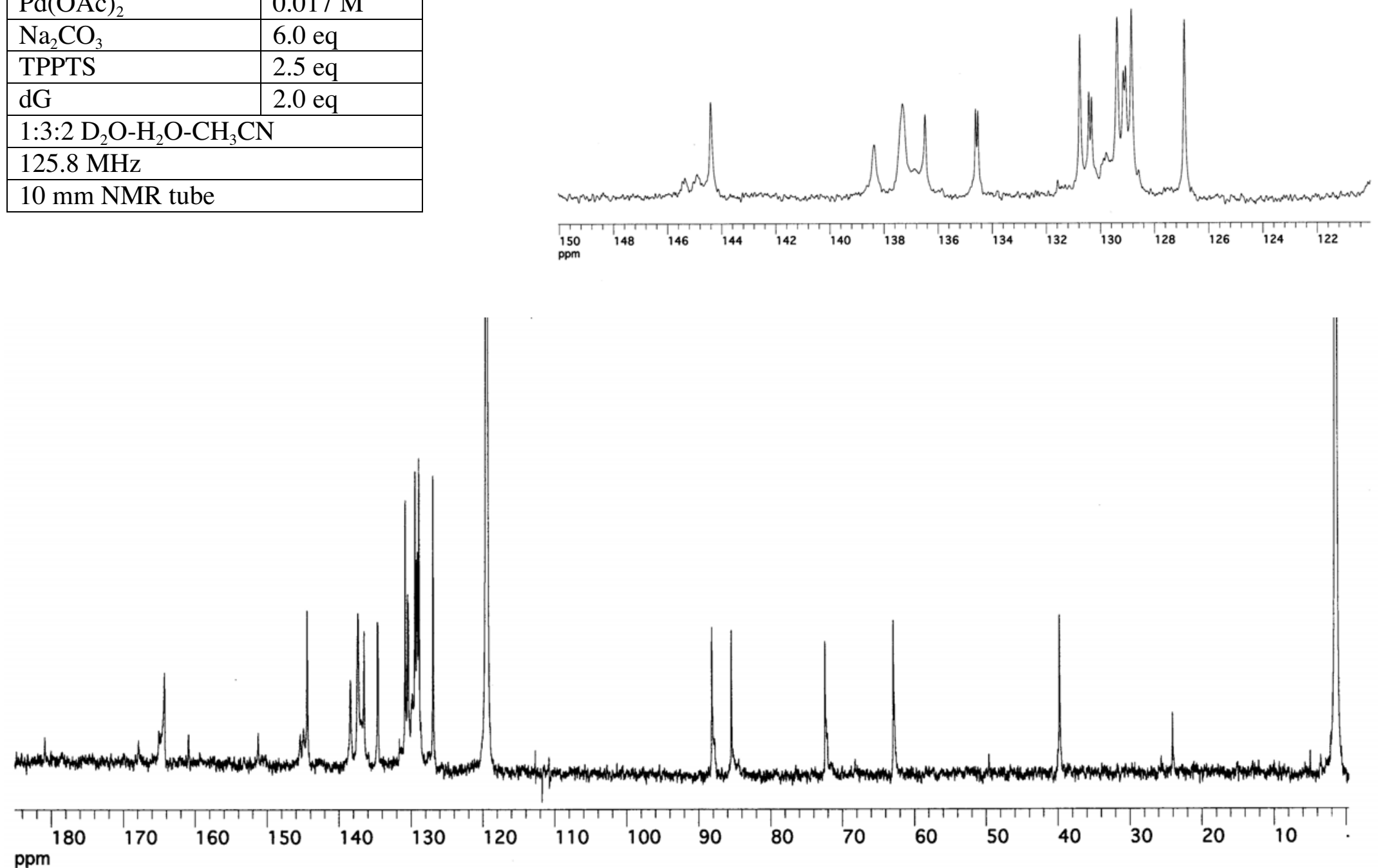


\begin{tabular}{|l|l|}
\hline $\mathrm{Pd}(\mathrm{OAc})_{2}$ & $0.007 \mathrm{M}$ \\
\hline $\mathrm{Na}_{2} \mathrm{CO}_{3}$ & $30.1 \mathrm{eq}$ \\
\hline TPPTS & $2.5 \mathrm{eq}$ \\
\hline $\mathrm{dG}$ & $10.3 \mathrm{eq}$ \\
\hline $1: 3: 2 \mathrm{D}_{2} \mathrm{O}-\mathrm{H}_{2} \mathrm{O}-\mathrm{CH}_{3} \mathrm{CN}$ \\
\hline $125.8 \mathrm{MHz}$ \\
\hline $10 \mathrm{~mm} \mathrm{NMR} \mathrm{tube}$ \\
\hline
\end{tabular}

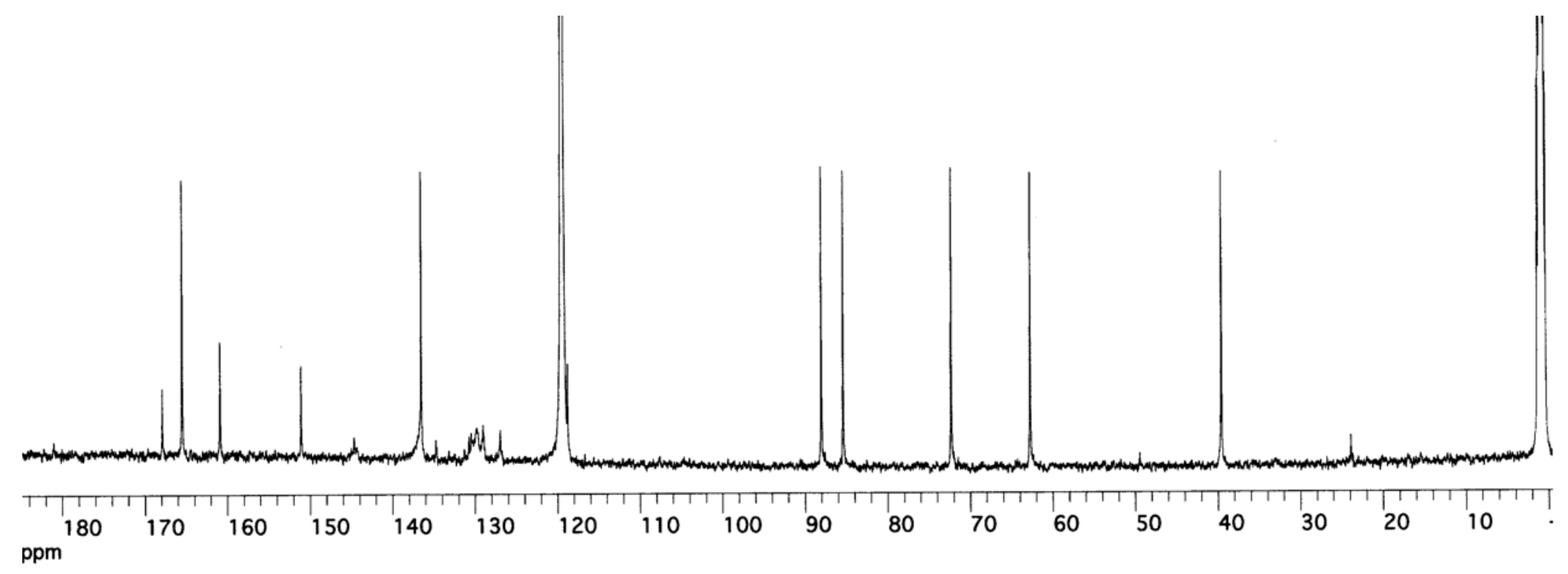

$\stackrel{n}{i}$ 


\begin{tabular}{|l|l|}
\hline $\mathrm{Pd}(\mathrm{OAc})_{2}$ & $0.017 \mathrm{M}$ \\
\hline $\mathrm{Na}_{2} \mathrm{CO}_{3}$ & $6.5 \mathrm{eq}$ \\
\hline TPPTS & $2.5 \mathrm{eq}$ \\
\hline $1 \mathrm{MedG}$ & $2.0 \mathrm{eq}$ \\
\hline $1: 3: 2 \mathrm{D}_{2} \mathrm{O}-\mathrm{H}_{2} \mathrm{O}-\mathrm{CH}_{3} \mathrm{CN}$ \\
\hline $125.8 \mathrm{MHz}$ \\
\hline $10 \mathrm{~mm} \mathrm{NMR} \mathrm{tube}$ \\
\hline
\end{tabular}
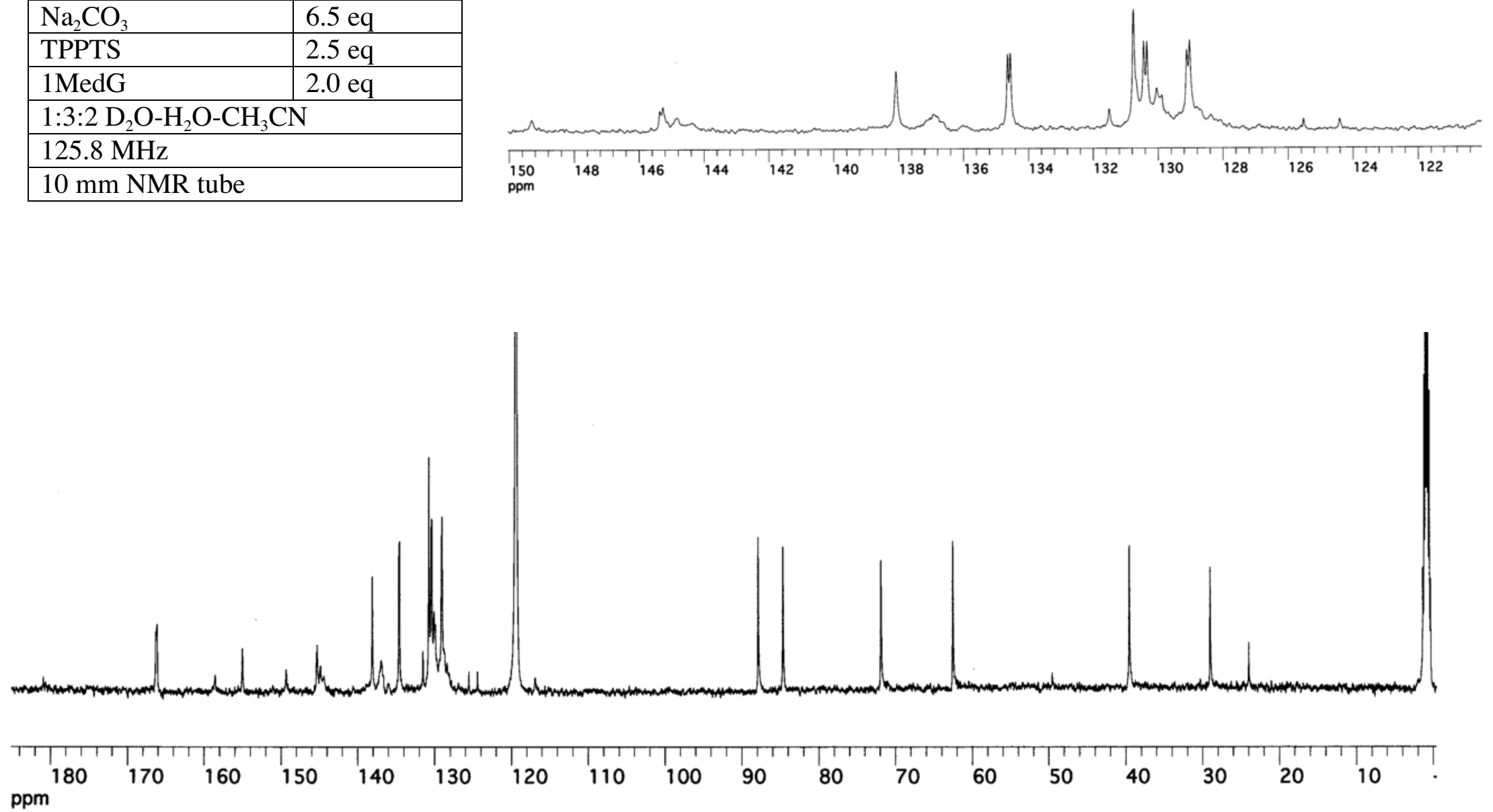

$\frac{n}{n}$ 


\begin{tabular}{|l|l|}
\hline $\mathrm{Pd}(\mathrm{OAc})_{2}$ & $0.017 \mathrm{M}$ \\
\hline $\mathrm{Na}_{2} \mathrm{CO}_{3}$ & $6.2 \mathrm{eq}$ \\
\hline $\mathrm{TPPTS}$ & $2.5 \mathrm{eq}$ \\
\hline $\mathrm{dA}$ & $2.0 \mathrm{eq}$ \\
\hline $1: 3: 2 \mathrm{D}_{2} \mathrm{O}-\mathrm{H}_{2} \mathrm{O}-\mathrm{CH}_{3} \mathrm{CN}$ \\
\hline $125.8 \mathrm{MHz}$ \\
\hline $10 \mathrm{~mm} \mathrm{NMR} \mathrm{tube}$ \\
\hline
\end{tabular}
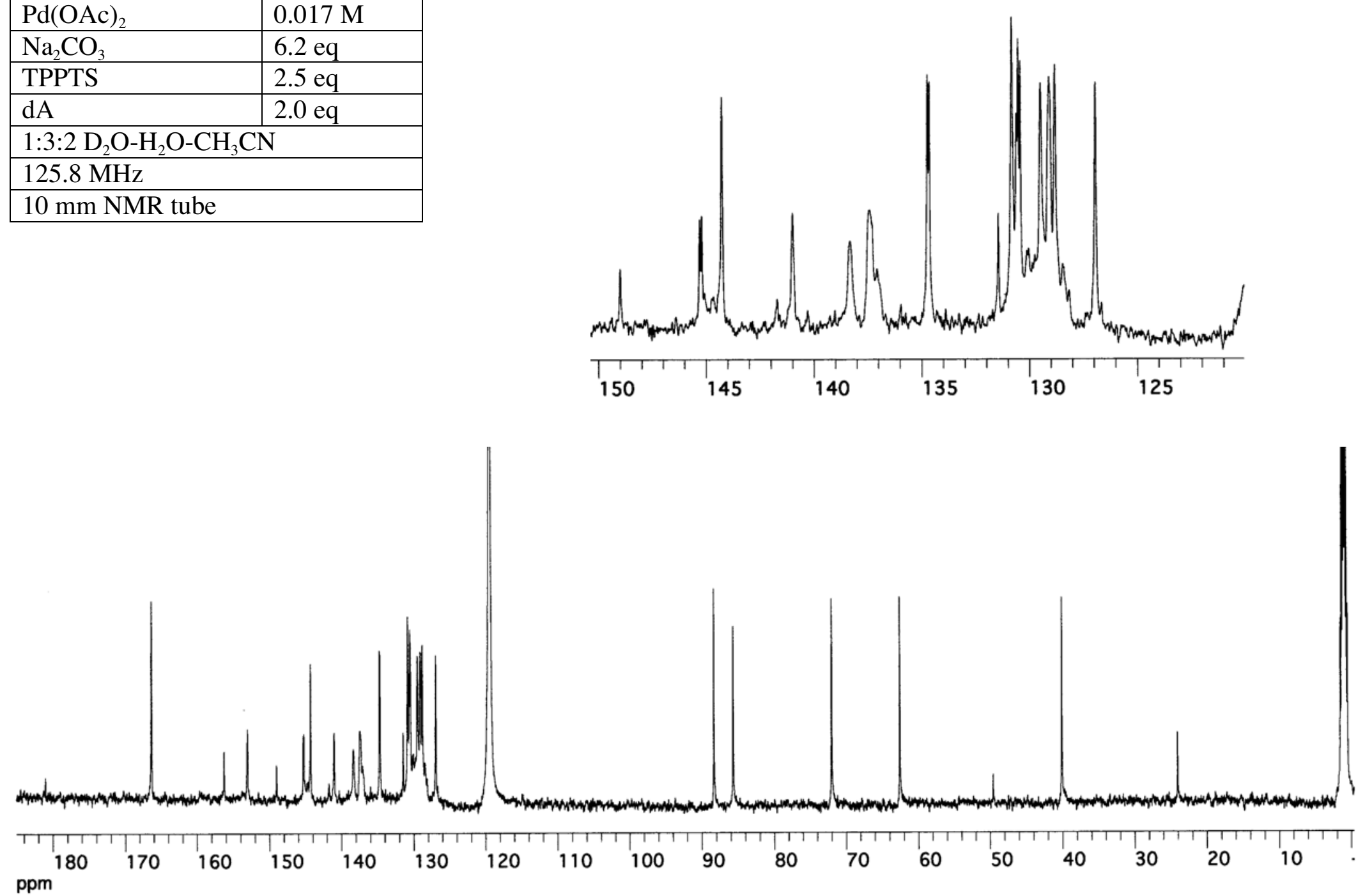

$\frac{n}{\sigma}$ 


\begin{tabular}{|l|l|}
\hline $\mathrm{Pd}(\mathrm{OAc})_{2}$ & $0.015 \mathrm{M}$ \\
\hline $\mathrm{Na}_{2} \mathrm{CO}_{3}$ & $24 \mathrm{eq}$ \\
\hline $\mathrm{TPPTS}$ & $2.5 \mathrm{eq}$ \\
\hline $\mathrm{PhB}(\mathrm{OH})_{2}$ & $15.3 \mathrm{eq}$ \\
\hline $2: 1 \mathrm{D}_{2} \mathrm{O}-\mathrm{CD}_{3} \mathrm{CN}$ \\
\hline $90.6 \mathrm{MHz}$ \\
\hline $5 \mathrm{~mm} \mathrm{NMR} \mathrm{tube}$ \\
\hline
\end{tabular}
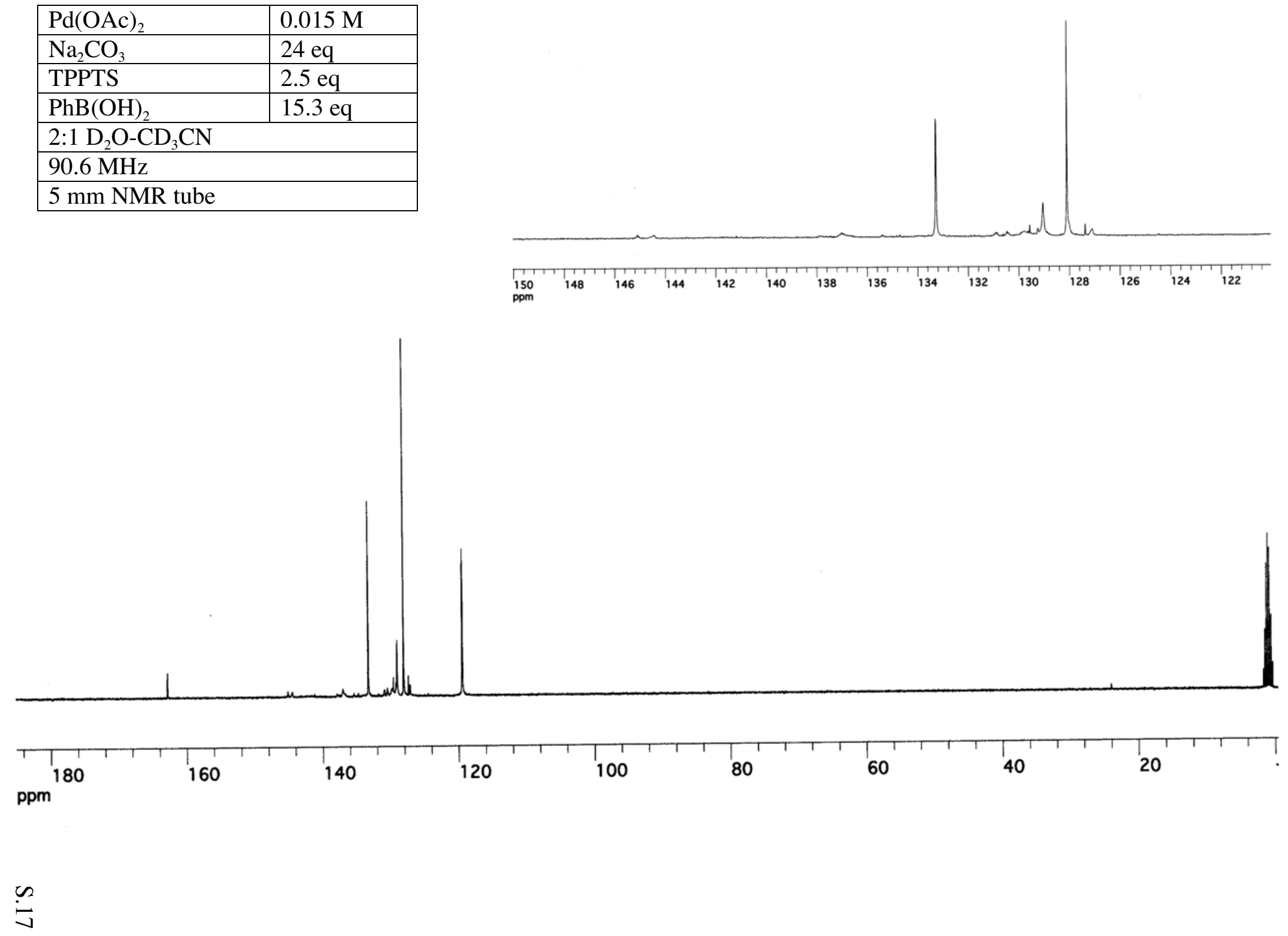


\begin{tabular}{|l|l|}
\hline $\mathrm{Pd}(\mathrm{OAc})_{2}$ & $0.017 \mathrm{M}$ \\
\hline $\mathrm{Na}_{2} \mathrm{CO}_{3}$ & $7.5 \mathrm{eq}$ \\
\hline $\mathrm{TPPTS}$ & $2.5 \mathrm{eq}$ \\
\hline $\mathrm{PhB}(\mathrm{OH})_{2}$ & $3.0 \mathrm{eq}$ \\
\hline $\mathrm{dG}$ & $2.1 \mathrm{eq}$ \\
\hline $1: 3: 2 \mathrm{D}_{2} \mathrm{O}-\mathrm{H}_{2} \mathrm{O}-\mathrm{CH}_{3} \mathrm{CN}$ \\
\hline $125.8 \mathrm{MHz}$ \\
\hline $10 \mathrm{~mm}$ tube \\
\hline
\end{tabular}

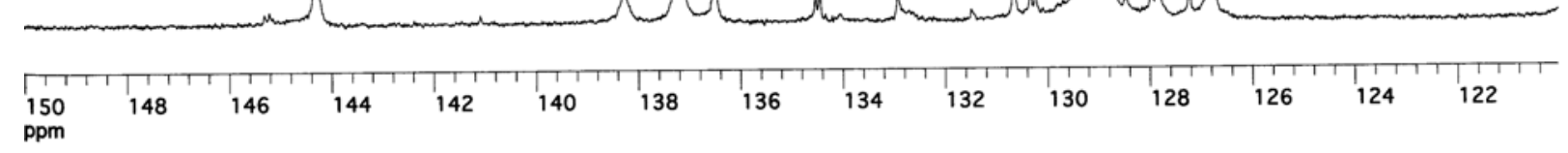

ppm

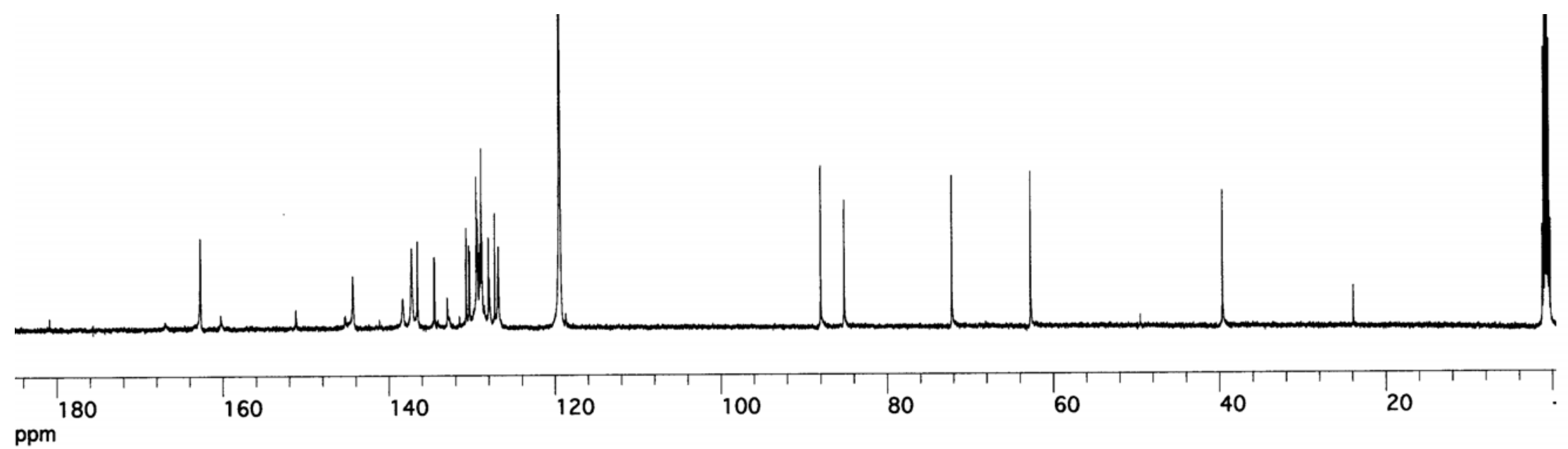




\begin{tabular}{|l|l|}
\hline $\mathrm{Pd}(\mathrm{OAc})_{2}$ & $0.017 \mathrm{M}$ \\
\hline $\mathrm{Na}_{2} \mathrm{CO}_{3}$ & $30.2 \mathrm{eq}$ \\
\hline $\mathrm{TPPTS}$ & $2.5 \mathrm{eq}$ \\
\hline $\mathrm{PhB}(\mathrm{OH})_{2}$ & $15.3 \mathrm{eq}$ \\
\hline $\mathrm{dG}$ & $10.0 \mathrm{eq}$ \\
\hline $1: 3: 2 \mathrm{D}_{2} \mathrm{O}-\mathrm{H}_{2} \mathrm{O}-\mathrm{CH}_{3} \mathrm{CN}$ \\
\hline $125.8 \mathrm{MHz}$ & \\
\hline $10 \mathrm{~mm}$ tube & \\
\hline
\end{tabular}
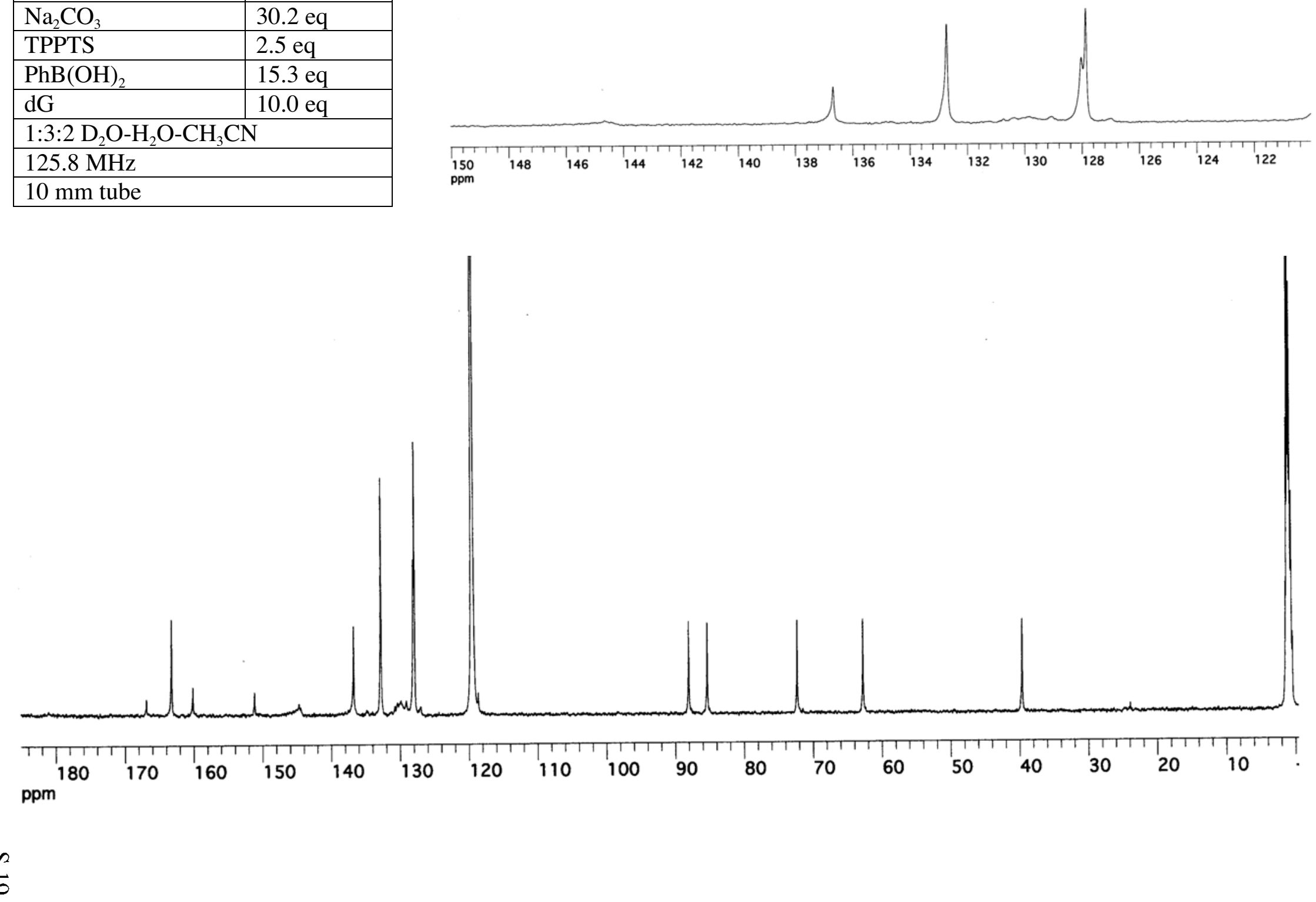


\begin{tabular}{|l|l|}
\hline $\mathrm{Pd}(\mathrm{OAc})_{2}$ & $0.016 \mathrm{M}$ \\
\hline $\mathrm{Na}_{2} \mathrm{CO}_{3}$ & $6.2 \mathrm{eq}$ \\
\hline $\mathrm{TPPTS}$ & $2.6 \mathrm{eq}$ \\
\hline $\mathrm{PhB}(\mathrm{OH})_{2}$ & $3.5 \mathrm{eq}$ \\
\hline $1 \mathrm{MedG}$ & $2.1 \mathrm{eq}$ \\
\hline $1: 3: 2 \mathrm{D}_{2} \mathrm{O}-\mathrm{H}_{2} \mathrm{O}-\mathrm{CH}_{3} \mathrm{CN}$ \\
\hline $125.8 \mathrm{MHz}$ & \\
\hline $10 \mathrm{~mm}$ tube & \\
\hline
\end{tabular}
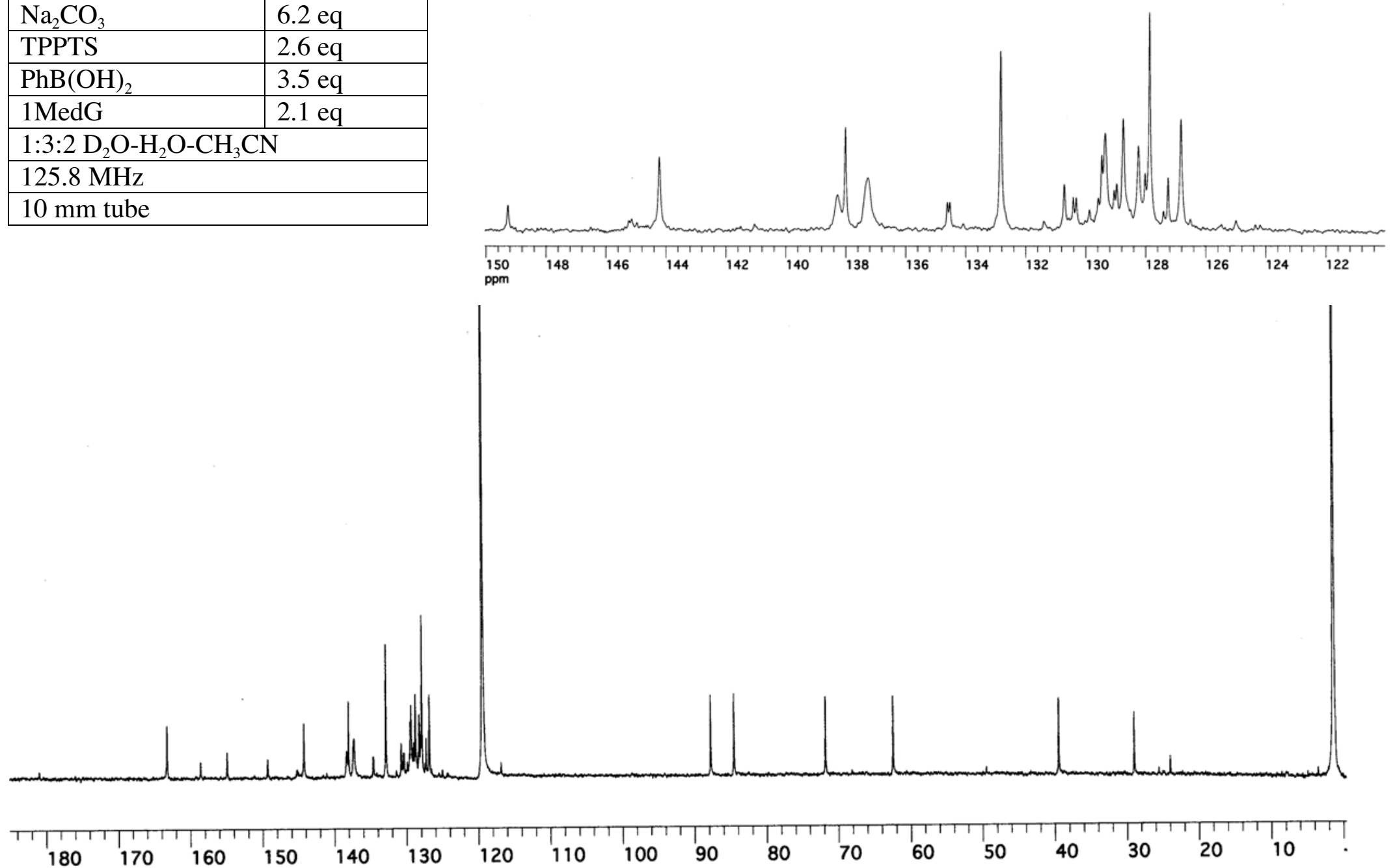
ppm 


\begin{tabular}{|l|l|}
\hline $\mathrm{Pd}(\mathrm{OAc})_{2}$ & $0.017 \mathrm{M}$ \\
\hline $\mathrm{Na}_{2} \mathrm{CO}_{3}$ & $6.4 \mathrm{eq}$ \\
\hline TPPTS & $2.5 \mathrm{eq}$ \\
\hline $\mathrm{PhB}(\mathrm{OH})_{2}$ & $3.0 \mathrm{eq}$ \\
\hline $\mathrm{dA}$ & $2.8 \mathrm{eq}$ \\
\hline $1: 3: 2 \mathrm{D}_{2} \mathrm{O}-\mathrm{H}_{2} \mathrm{O}-\mathrm{CH}_{3} \mathrm{CN}$ \\
\hline $125.8 \mathrm{MHz}$ \\
\hline $10 \mathrm{~mm}$ tube \\
\hline
\end{tabular}
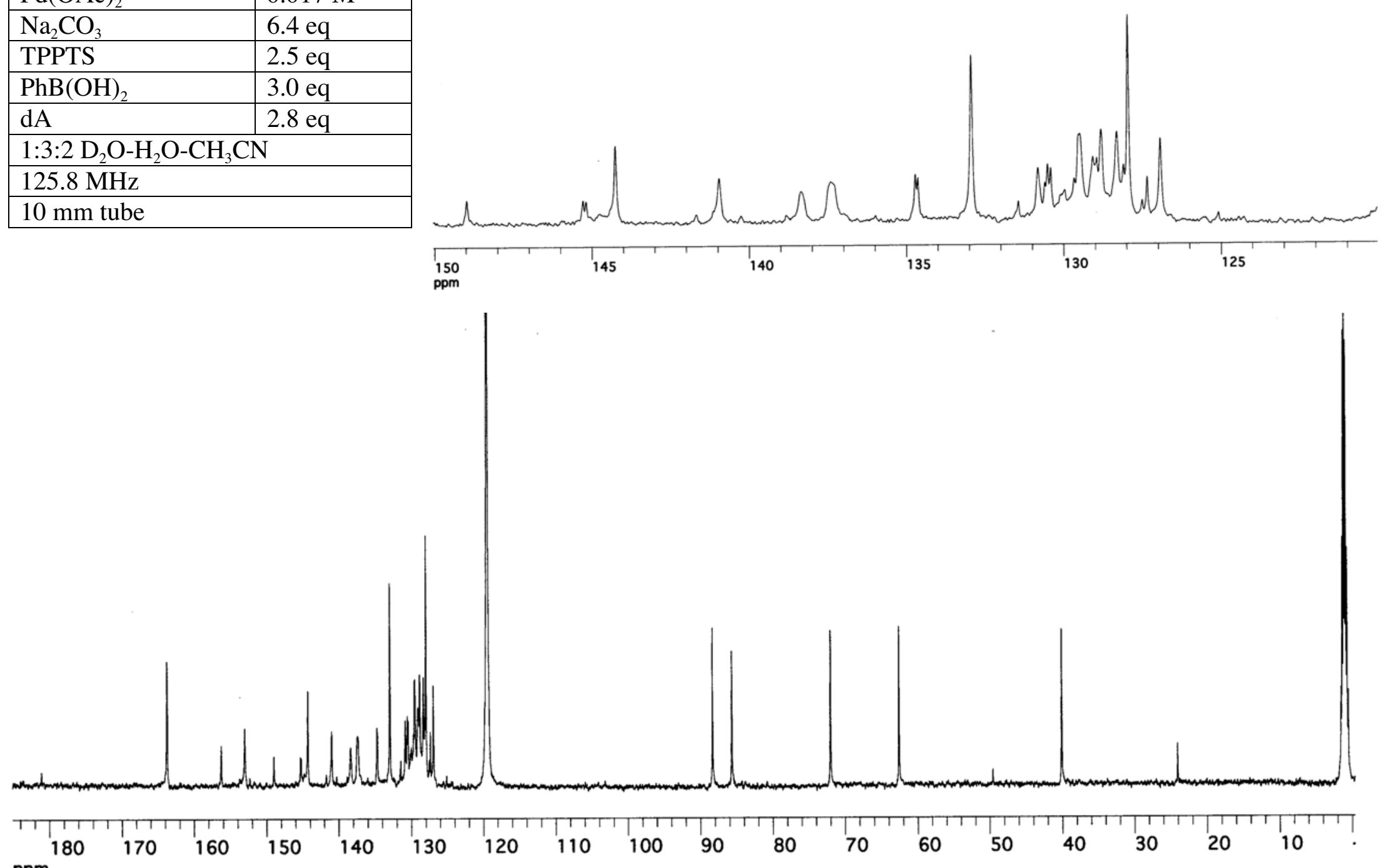

ppm 

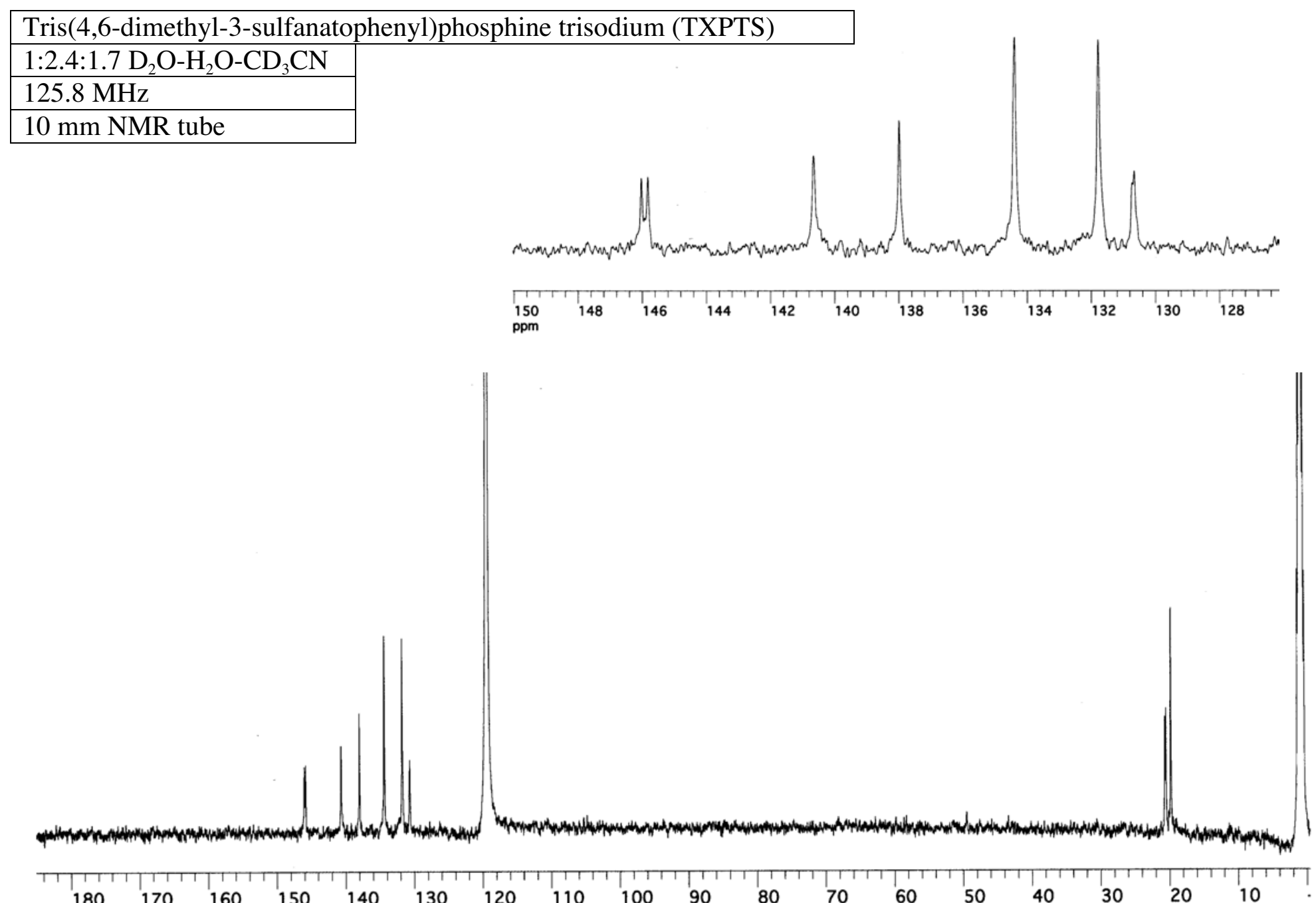

ppm 


\begin{tabular}{|l|l|}
\hline TXPTS oxide (TXPTS $+\mathrm{H}_{2} \mathrm{O}_{2}$ ) & $0.02 \mathrm{M}$ \\
\hline $2: 1 \mathrm{D}_{2} \mathrm{O}-\mathrm{CH}_{3} \mathrm{CN}$ & \\
\hline $125.8 \mathrm{MHz}$ \\
\hline $10 \mathrm{~mm} \mathrm{NMR} \mathrm{tube}$ \\
\hline
\end{tabular}
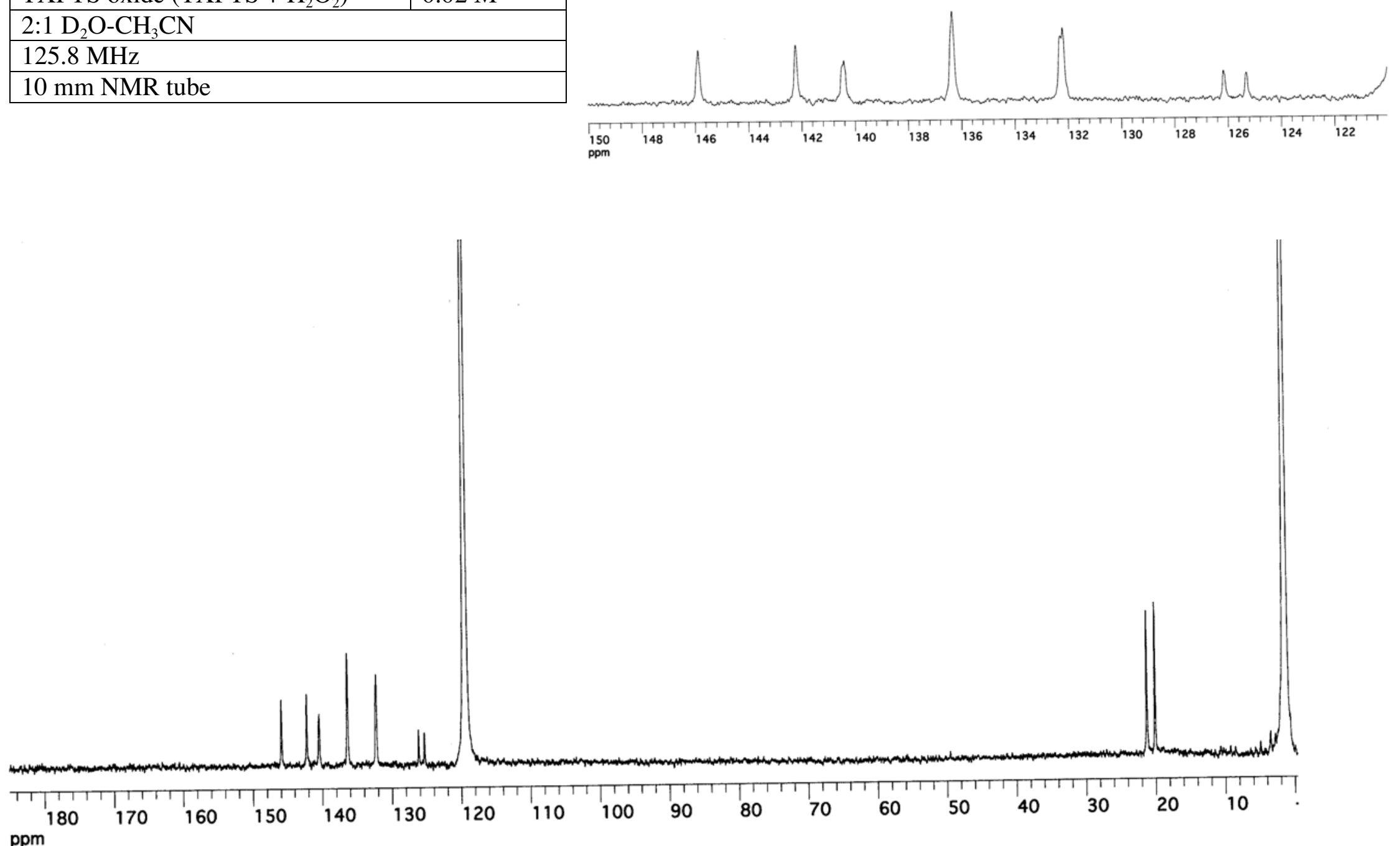


\begin{tabular}{|l|l|}
\hline $\mathrm{Pd}(\mathrm{OAc})_{2}$ & $0.017 \mathrm{M}$ \\
\hline TXPTS & $2.4 \mathrm{eq}$ \\
\hline $1: 2.4: 1.7 \mathrm{D}_{2} \mathrm{O}-\mathrm{H}_{2} \mathrm{O}-\mathrm{CD}_{3} \mathrm{CN}$ \\
\hline $125.8 \mathrm{MHz}$ \\
\hline $10 \mathrm{~mm} \mathrm{NMR}$ tube \\
\hline
\end{tabular}
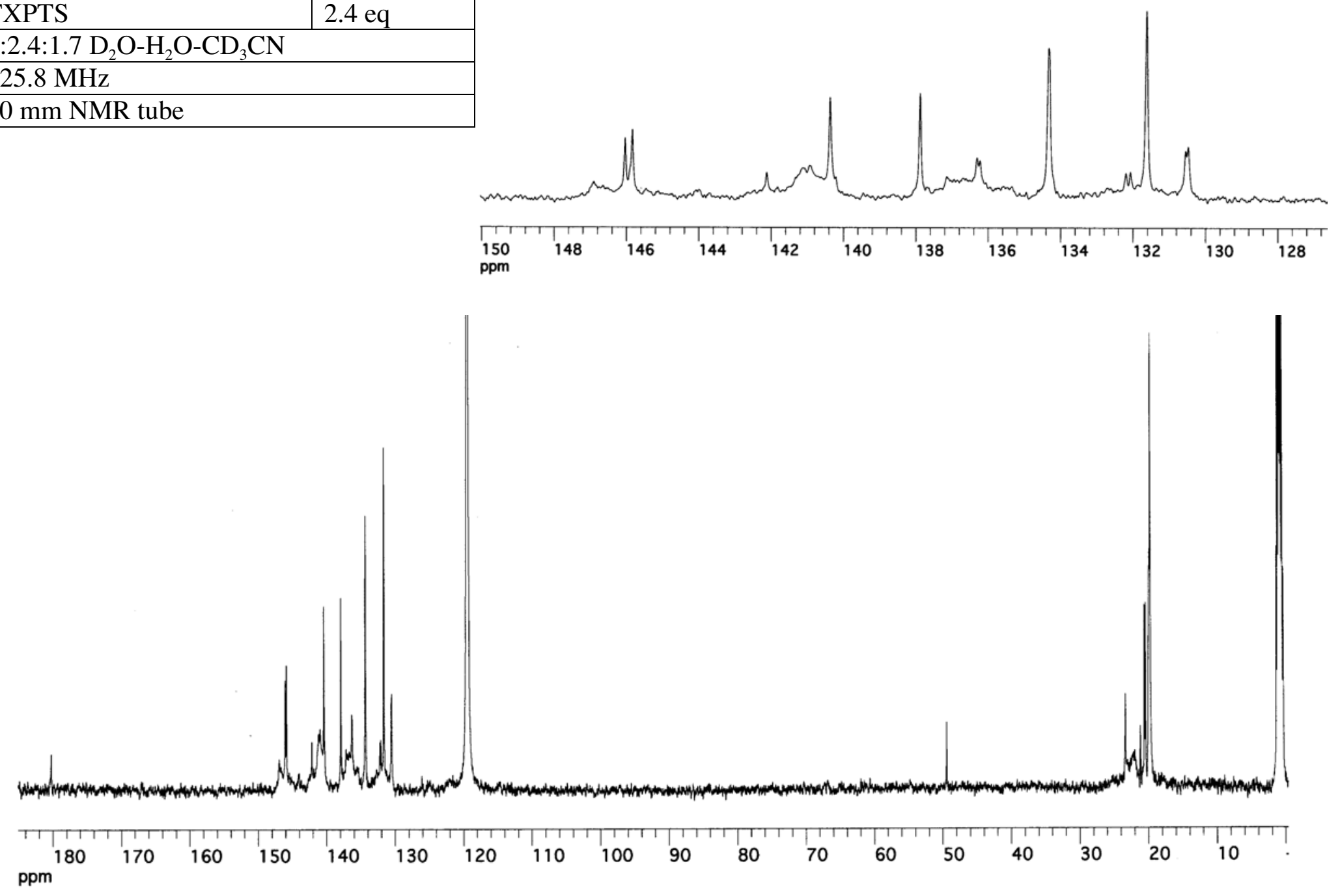


\begin{tabular}{|l|l|}
\hline $\mathrm{Pd}(\mathrm{OAc})_{2}$ & $0.01 \mathrm{M}$ \\
\hline $\mathrm{Na}_{2} \mathrm{CO}_{3}$ & $6.0 \mathrm{eq}$ \\
\hline TXPTS & $2.6 \mathrm{eq}$ \\
\hline $2: 1 \mathrm{D}_{2} \mathrm{O}-\mathrm{CH}_{3} \mathrm{CN}$ & \\
\hline $90.6 \mathrm{MHz}$ & \\
\hline $5 \mathrm{~mm} \mathrm{NMR}$ tube & \\
\hline
\end{tabular}
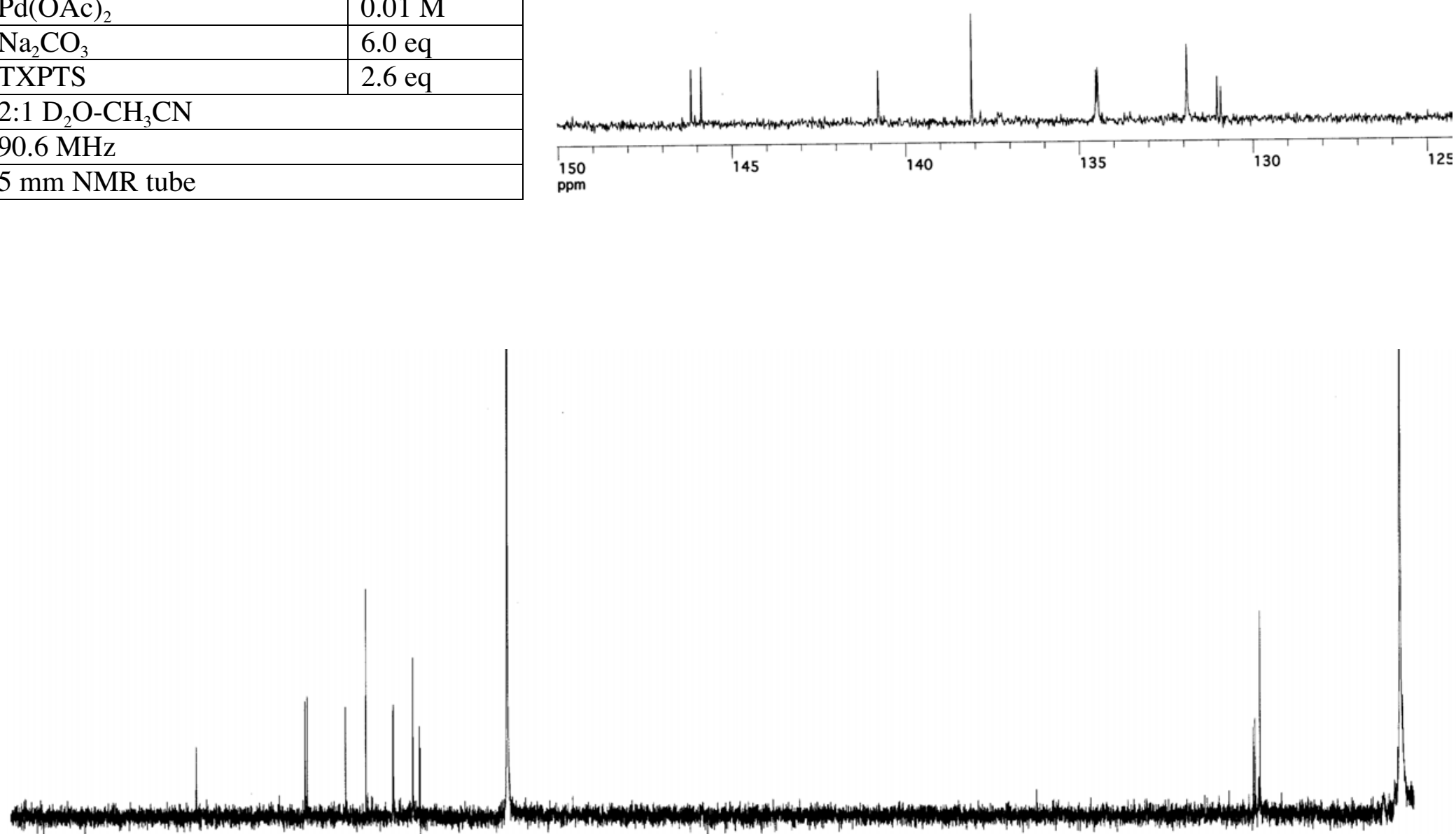

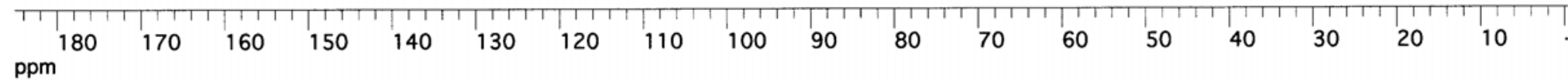




\begin{tabular}{|l|l|}
\hline $\mathrm{Pd}(\mathrm{OAc})_{2}$ & $0.02 \mathrm{M}$ \\
\hline $\mathrm{Na}_{2} \mathrm{CO}_{3}$ & $6.2 \mathrm{eq}$ \\
\hline TXPTS & $2.8 \mathrm{eq}$ \\
\hline $\mathrm{dG}$ & $2.1 \mathrm{eq}$ \\
\hline $2: 1 \mathrm{H}_{2} \mathrm{O}-\mathrm{CH}_{3} \mathrm{CN}$ \\
\hline $125.8 \mathrm{MHz}$ \\
\hline $10 \mathrm{~mm} \mathrm{NMR} \mathrm{tube}$ \\
\hline
\end{tabular}
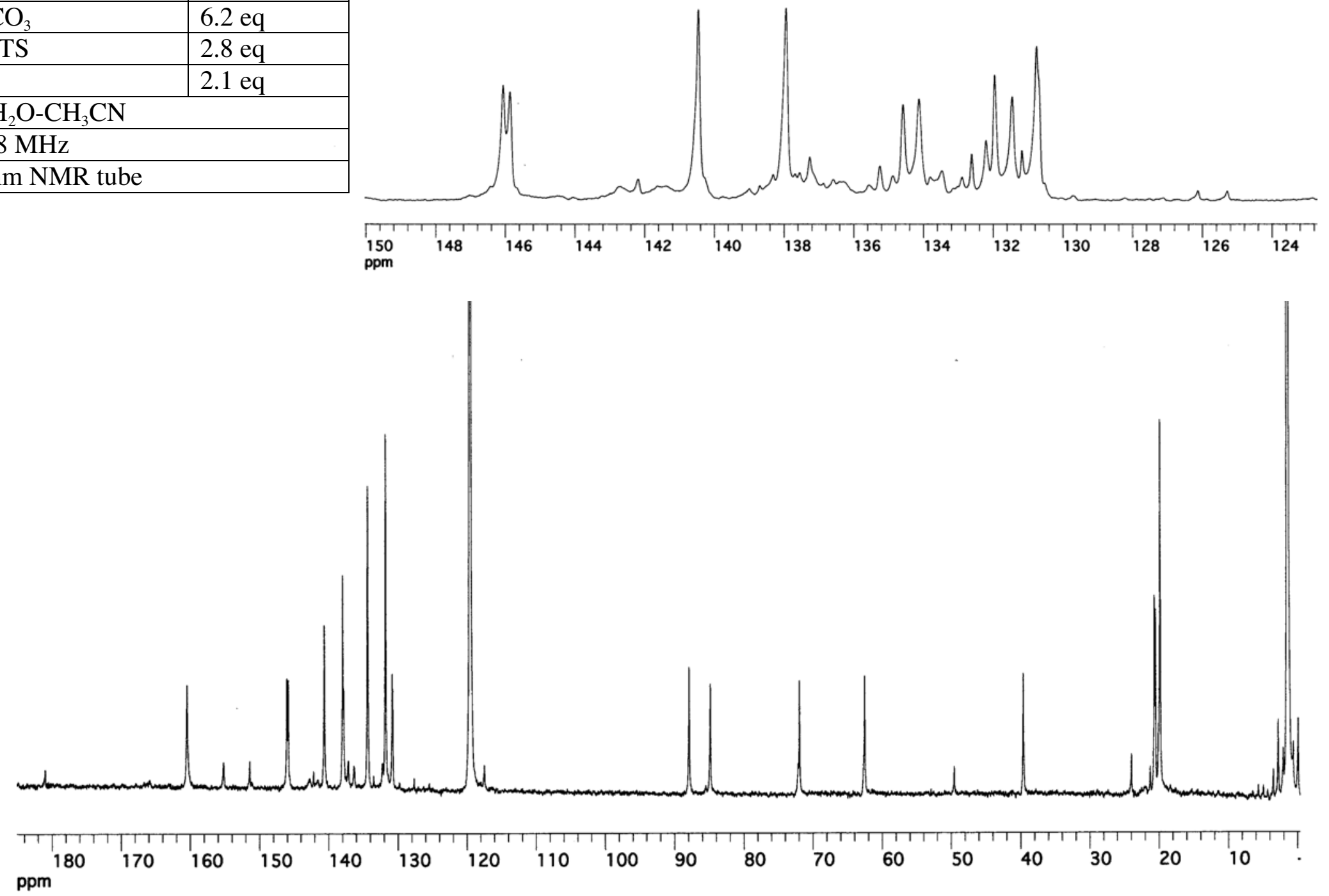


\begin{tabular}{|l|l|}
\hline $\mathrm{Pd}(\mathrm{OAc})_{2}$ & $0.017 \mathrm{M}$ \\
\hline $\mathrm{Na}_{2} \mathrm{CO}_{3}$ & $6.2 \mathrm{eq}$ \\
\hline TXPTS & $2.5 \mathrm{eq}$ \\
\hline $1 \mathrm{MedG}$ & $2.0 \mathrm{eq}$ \\
\hline $1: 3: 2 \mathrm{D}_{2} \mathrm{O}-\mathrm{H}_{2} \mathrm{O}-\mathrm{CD}_{3} \mathrm{CN}$ \\
\hline $125.8 \mathrm{MHz}$ \\
\hline $10 \mathrm{~mm} \mathrm{NMR}$ tube \\
\hline
\end{tabular}
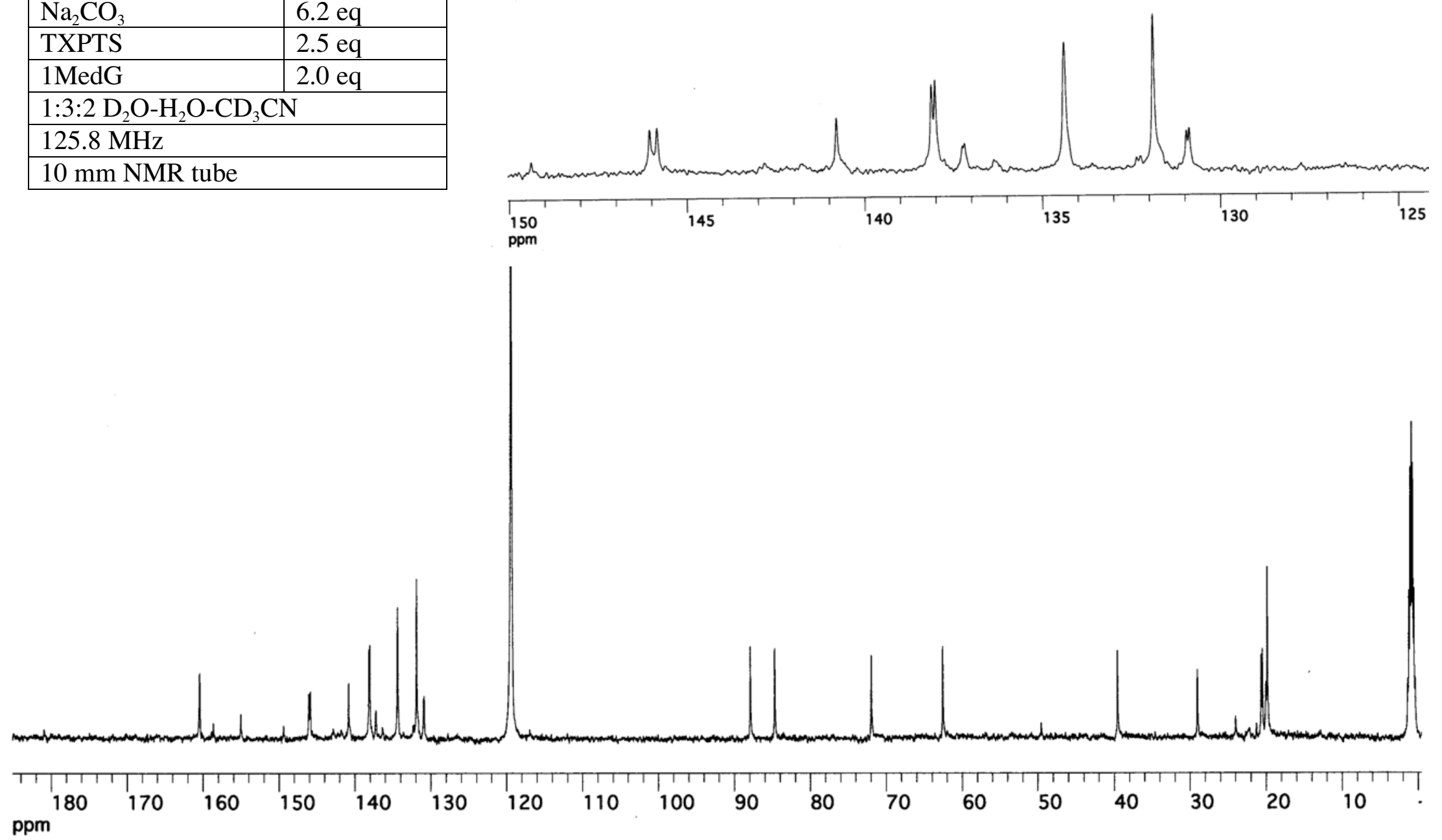

N 


\begin{tabular}{|l|l|}
\hline $\mathrm{Pd}(\mathrm{OAc})_{2}$ & $0.017 \mathrm{M}$ \\
\hline $\mathrm{Na}_{2} \mathrm{CO}_{3}$ & $7.3 \mathrm{eq}$ \\
\hline TXPTS & $2.5 \mathrm{eq}$ \\
\hline $\mathrm{dA}$ & $2.0 \mathrm{eq}$ \\
\hline $1: 3: 2 \mathrm{D}_{2} \mathrm{O}-\mathrm{H}_{2} \mathrm{O}-\mathrm{CD}_{3} \mathrm{CN}$ \\
\hline $125.8 \mathrm{MHz}$ \\
\hline $10 \mathrm{~mm} \mathrm{NMR} \mathrm{tube}$ \\
\hline
\end{tabular}

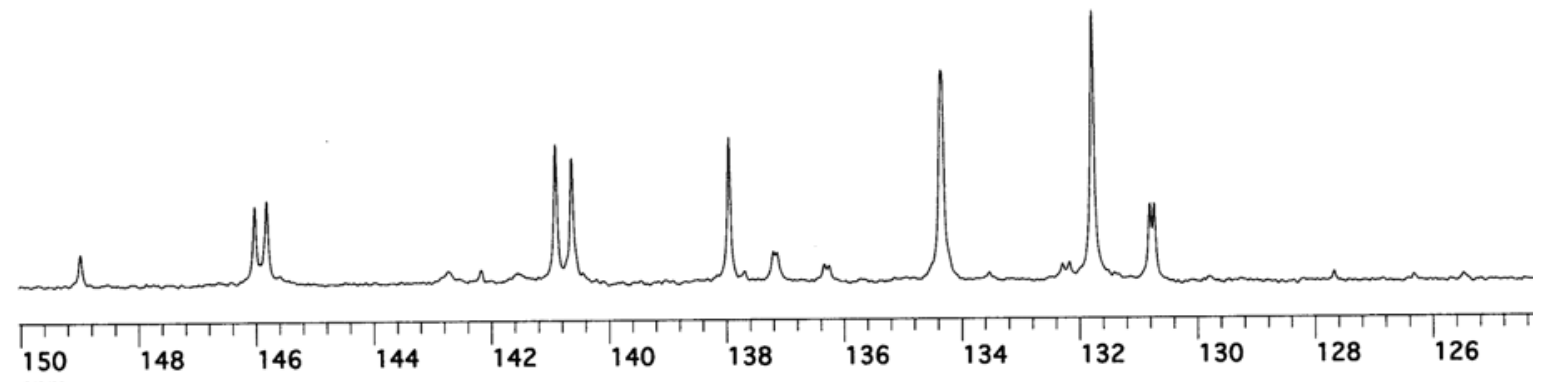
150
ppm

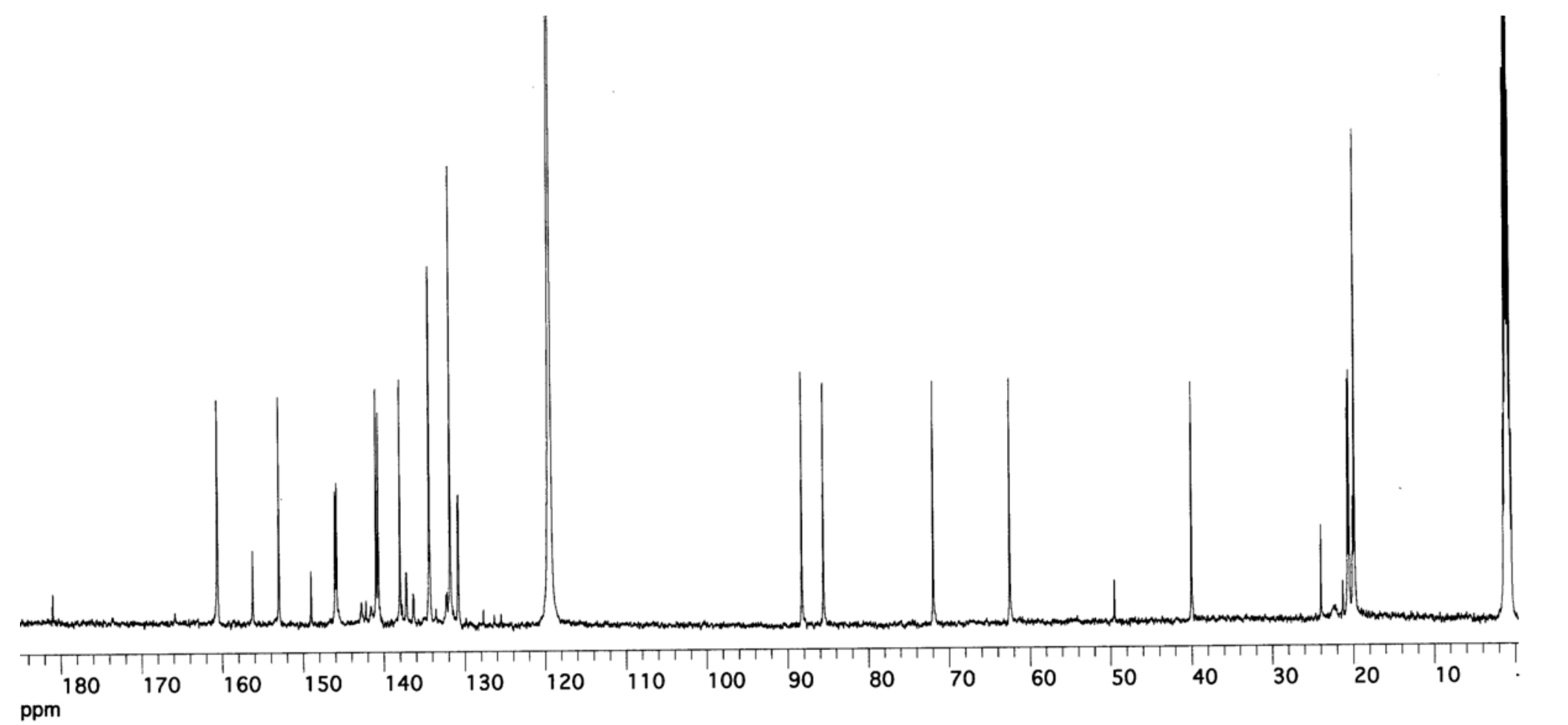




\begin{tabular}{|l|l|}
\hline $\mathrm{Pd}(\mathrm{OAc})_{2}$ & $0.014 \mathrm{M}$ \\
\hline $\mathrm{Na}_{2} \mathrm{CO}_{3}$ & $9.0 \mathrm{eq}$ \\
\hline $\mathrm{TXPTS}$ & $3.3 \mathrm{eq}$ \\
\hline $\mathrm{PhB}(\mathrm{OH})_{2}$ & $5.4 \mathrm{eq}$ \\
\hline $1: 3: 2 \mathrm{D}_{2} \mathrm{O}-\mathrm{H}_{2} \mathrm{O}-\mathrm{CD}_{3} \mathrm{CN}$ \\
\hline $125.8 \mathrm{MHz}$ \\
\hline $10 \mathrm{~mm} \mathrm{NMR} \mathrm{tube}$ \\
\hline
\end{tabular}
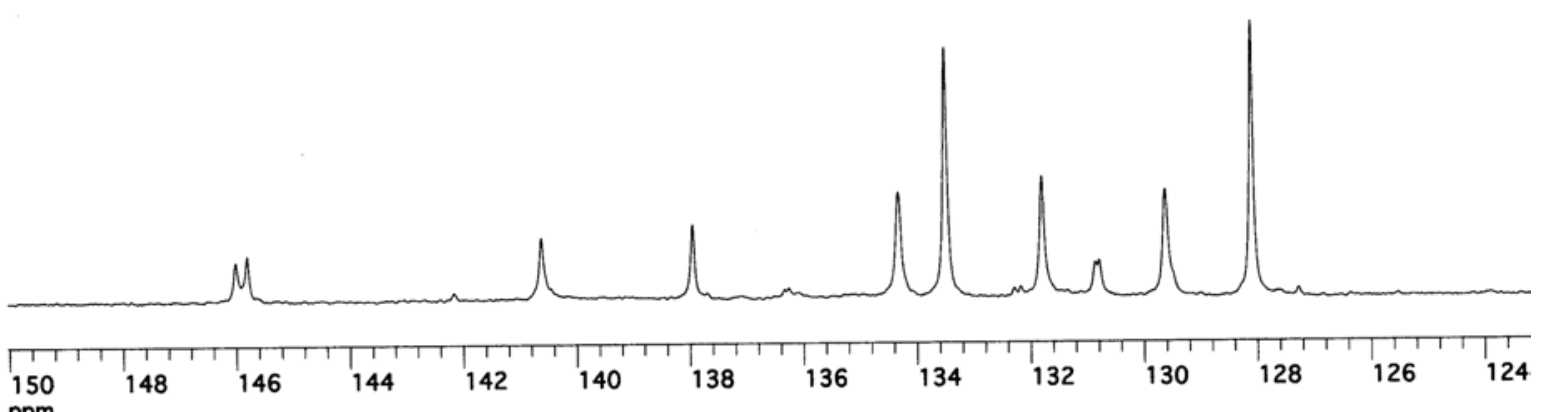

150
ppm

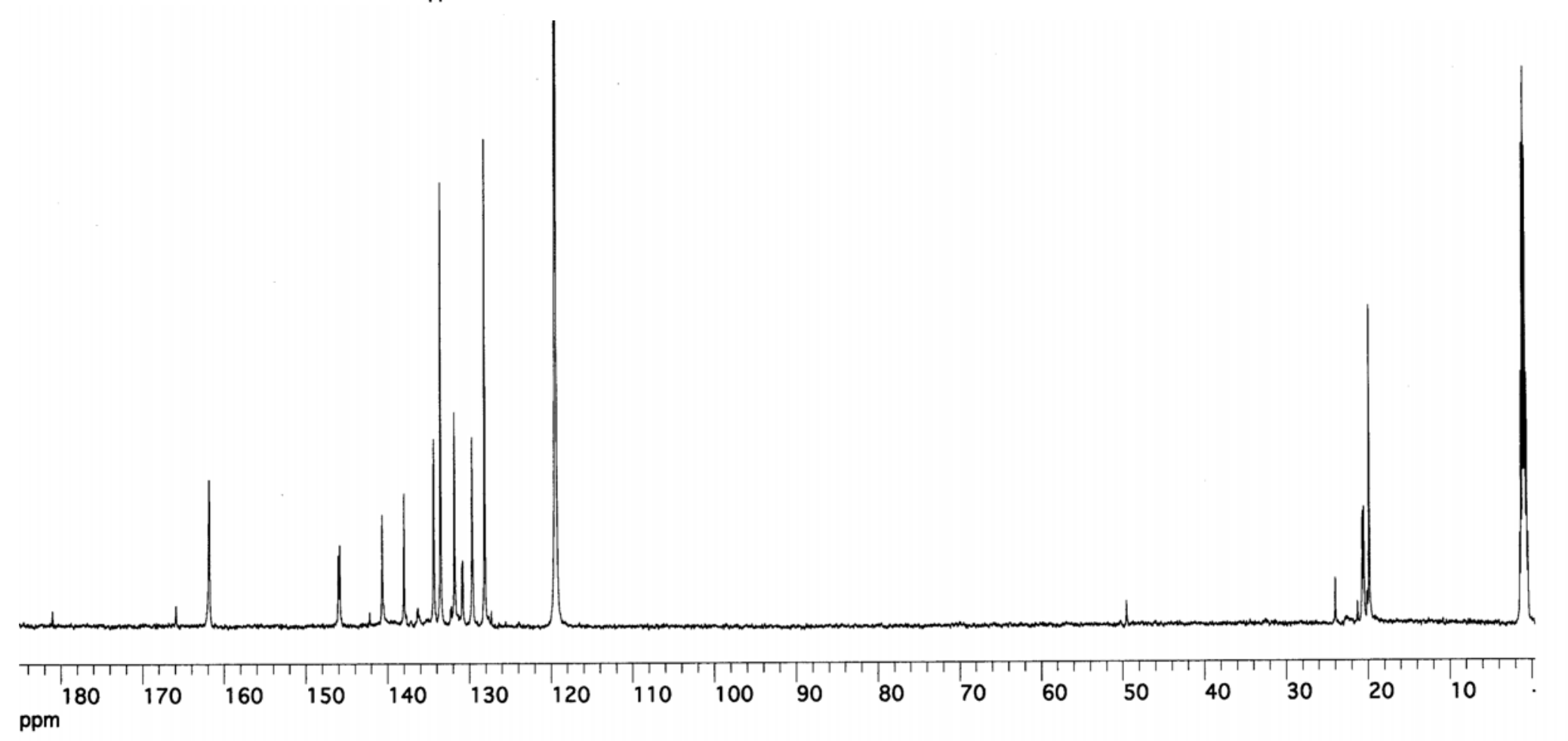




\begin{tabular}{|l|l|}
\hline $\mathrm{Pd}(\mathrm{OAc})_{2}$ & $0.017 \mathrm{M}$ \\
\hline $\mathrm{Na}_{2} \mathrm{CO}_{3}$ & $6.2 \mathrm{eq}$ \\
\hline $\mathrm{TXPTS}$ & $2.5 \mathrm{eq}$ \\
\hline $\mathrm{PhB}(\mathrm{OH})_{2}$ & $4.0 \mathrm{eq}$ \\
\hline $\mathrm{dG}$ & $2.0 \mathrm{eq}$ \\
\hline $1: 3: 2 \mathrm{D}_{2} \mathrm{O}-\mathrm{H}_{2} \mathrm{O}-\mathrm{CH}_{3} \mathrm{CN}$ \\
\hline $125.8 \mathrm{MHz}$ \\
\hline $10 \mathrm{~mm} \mathrm{NMR}$ tube \\
\hline
\end{tabular}
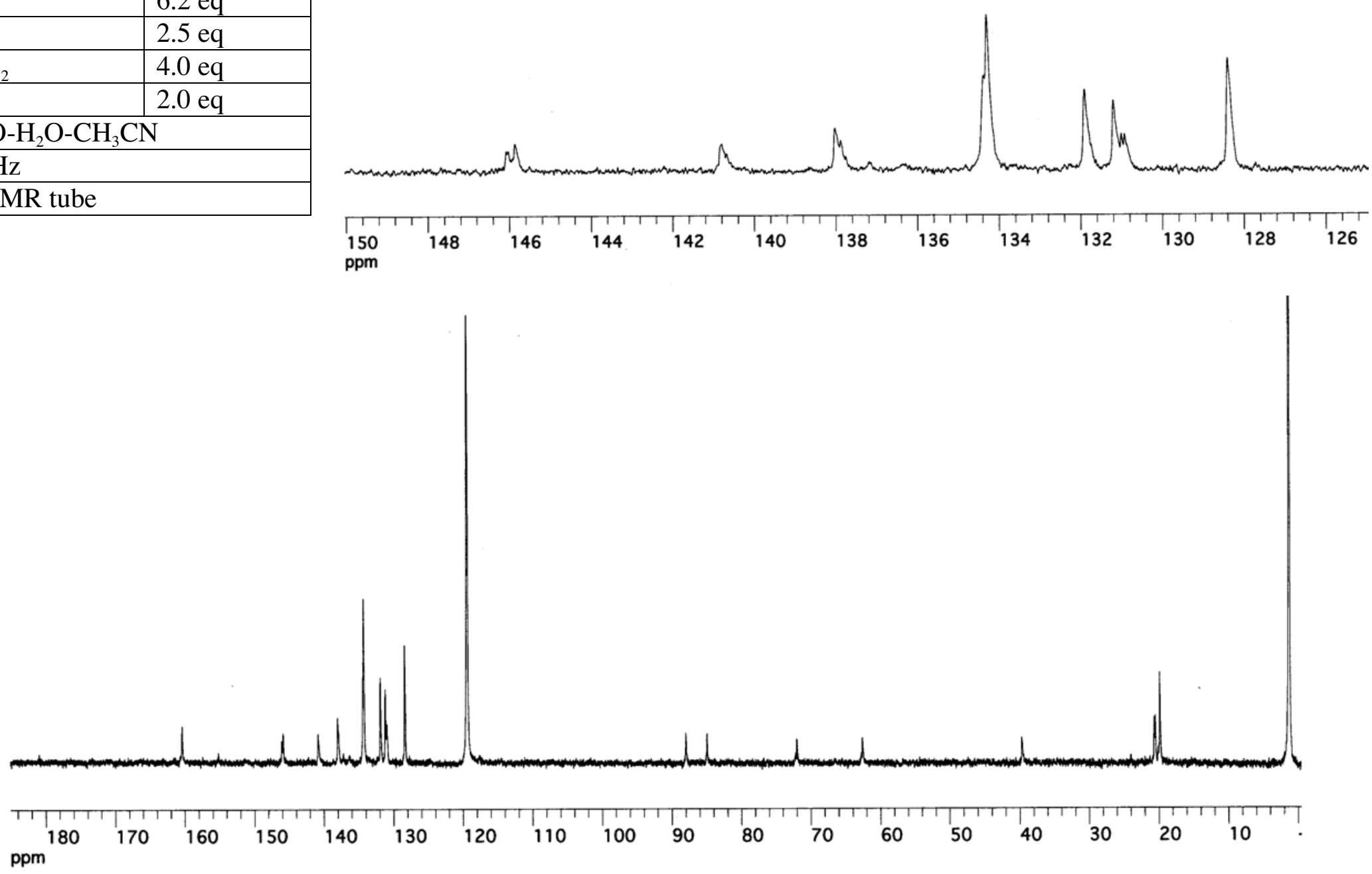


\begin{tabular}{|l|l|}
\hline $\mathrm{Pd}(\mathrm{OAc})_{2}$ & $0.017 \mathrm{M}$ \\
\hline $\mathrm{Na}_{2} \mathrm{CO}_{3}$ & $6.1 \mathrm{eq}$ \\
\hline TXPTS & $2.5 \mathrm{eq}$ \\
\hline $\mathrm{PhB}(\mathrm{OH})_{2}$ & $3.1 \mathrm{eq}$ \\
\hline $1 \mathrm{MedG}$ & $2.0 \mathrm{eq}$ \\
\hline $1: 3: 2 \mathrm{D}_{2} \mathrm{O}-\mathrm{H}_{2} \mathrm{O}-\mathrm{CD}_{3} \mathrm{CN}$ \\
\hline $125.8 \mathrm{MHz}$ \\
\hline $10 \mathrm{~mm} \mathrm{NMR}$ tube \\
\hline
\end{tabular}
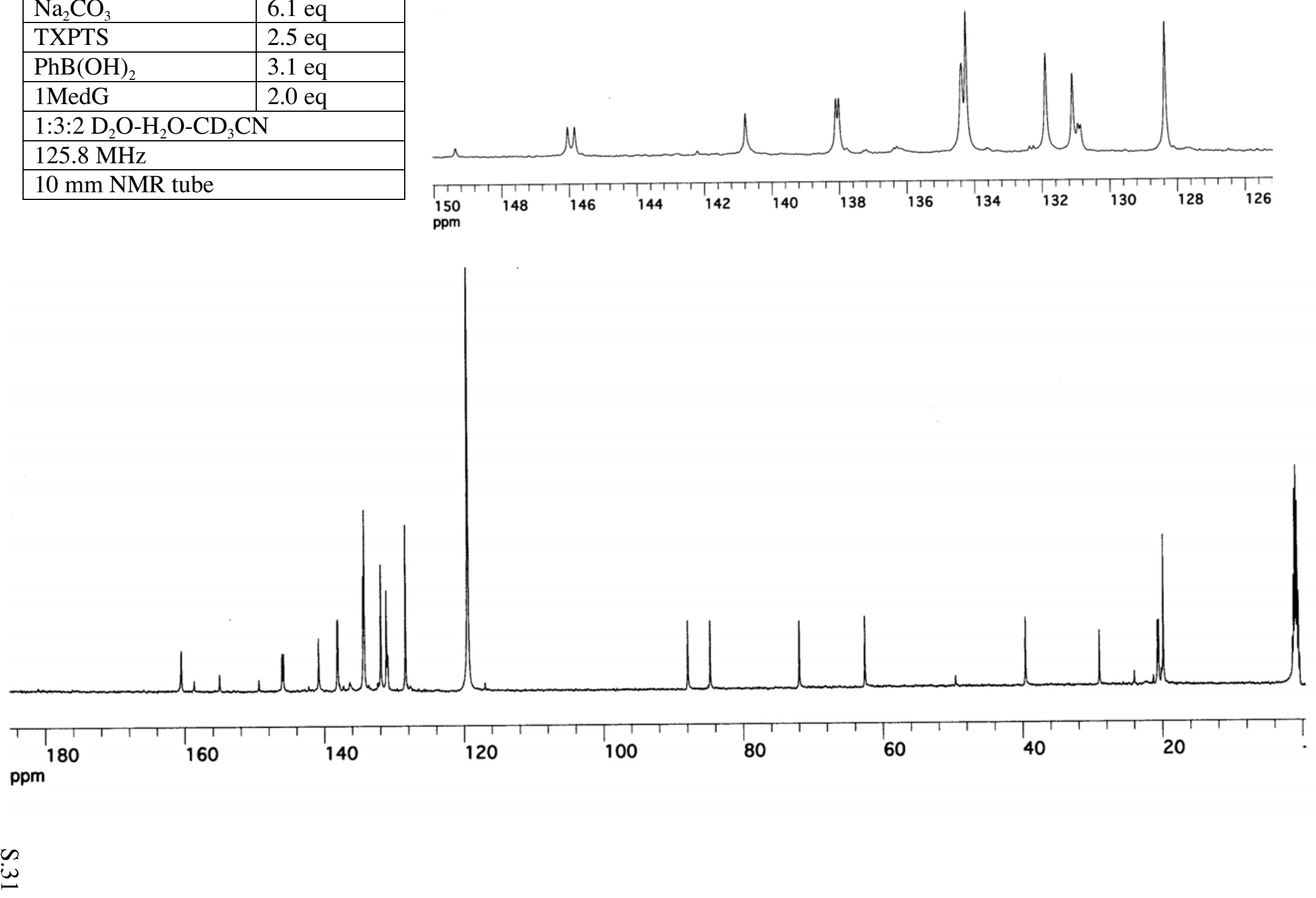


\begin{tabular}{|l|l|}
\hline $\mathrm{Pd}(\mathrm{OAc})_{2}$ & $0.01 \mathrm{M}$ \\
\hline $\mathrm{Na}_{2} \mathrm{CO}_{3}$ & $6.2 \mathrm{eq}$ \\
\hline $\mathrm{TXPTS}$ & $2.5 \mathrm{eq}$ \\
\hline $\mathrm{PhB}(\mathrm{OH})_{2}$ & $16.5 \mathrm{eq}$ \\
\hline $\mathrm{dA}$ & 10.3 \\
\hline $2: 1 \mathrm{D}_{2} \mathrm{O}-\mathrm{CH}_{3} \mathrm{CN}$ & \\
\hline $90.6 \mathrm{MHz}$ & \\
\hline $5 \mathrm{~mm} \mathrm{NMR}$ tube \\
\hline
\end{tabular}
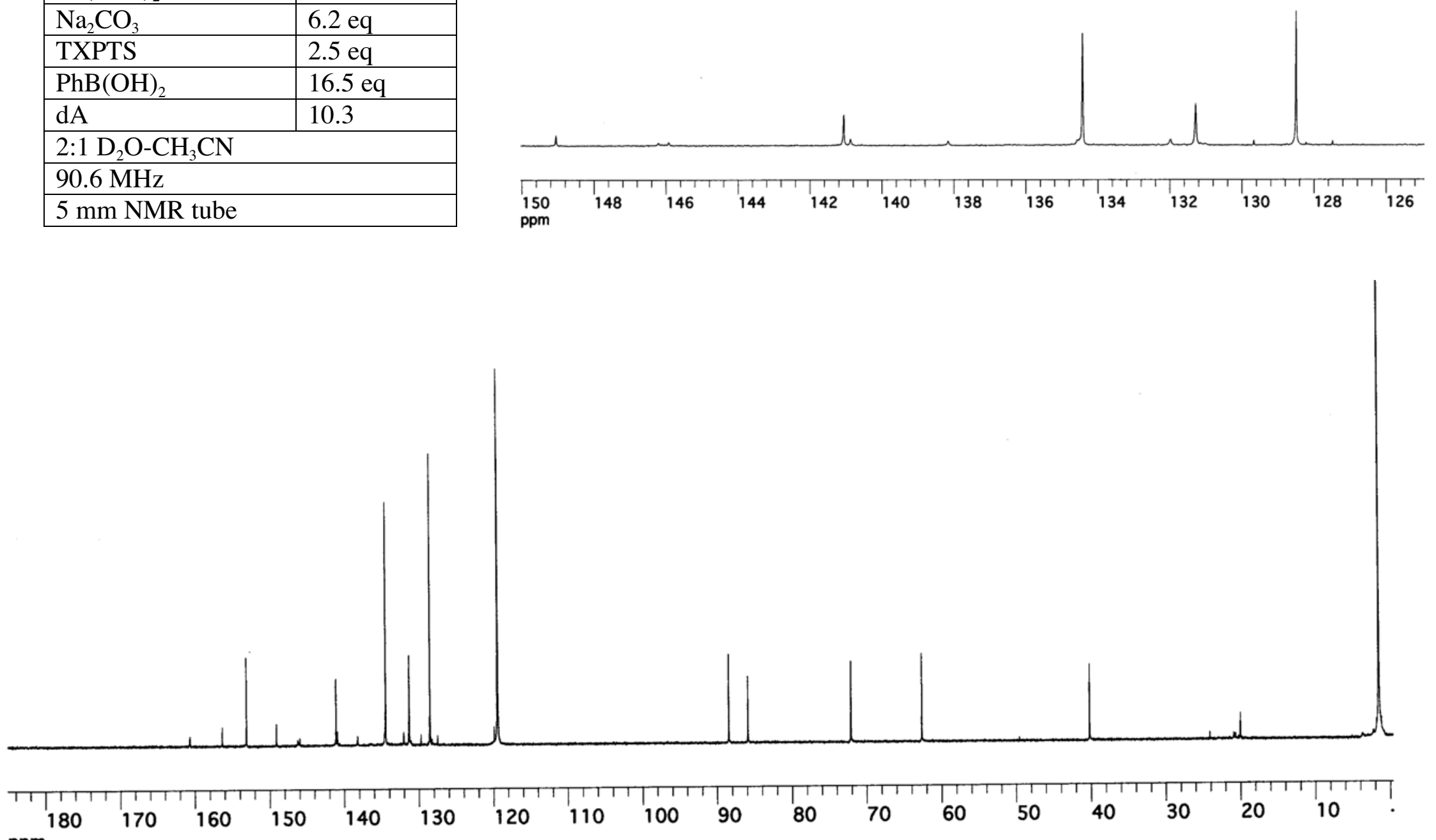
ppm 


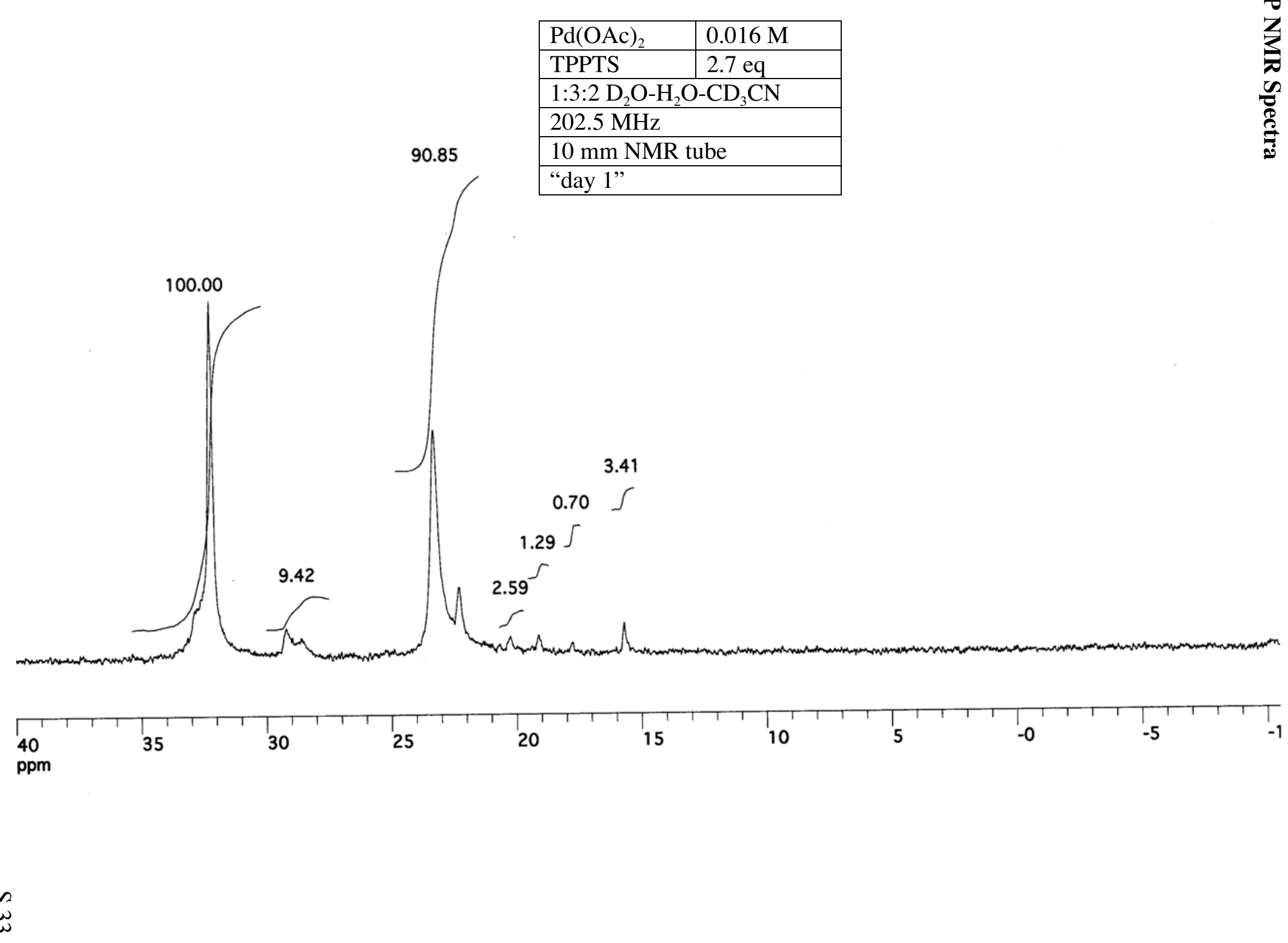




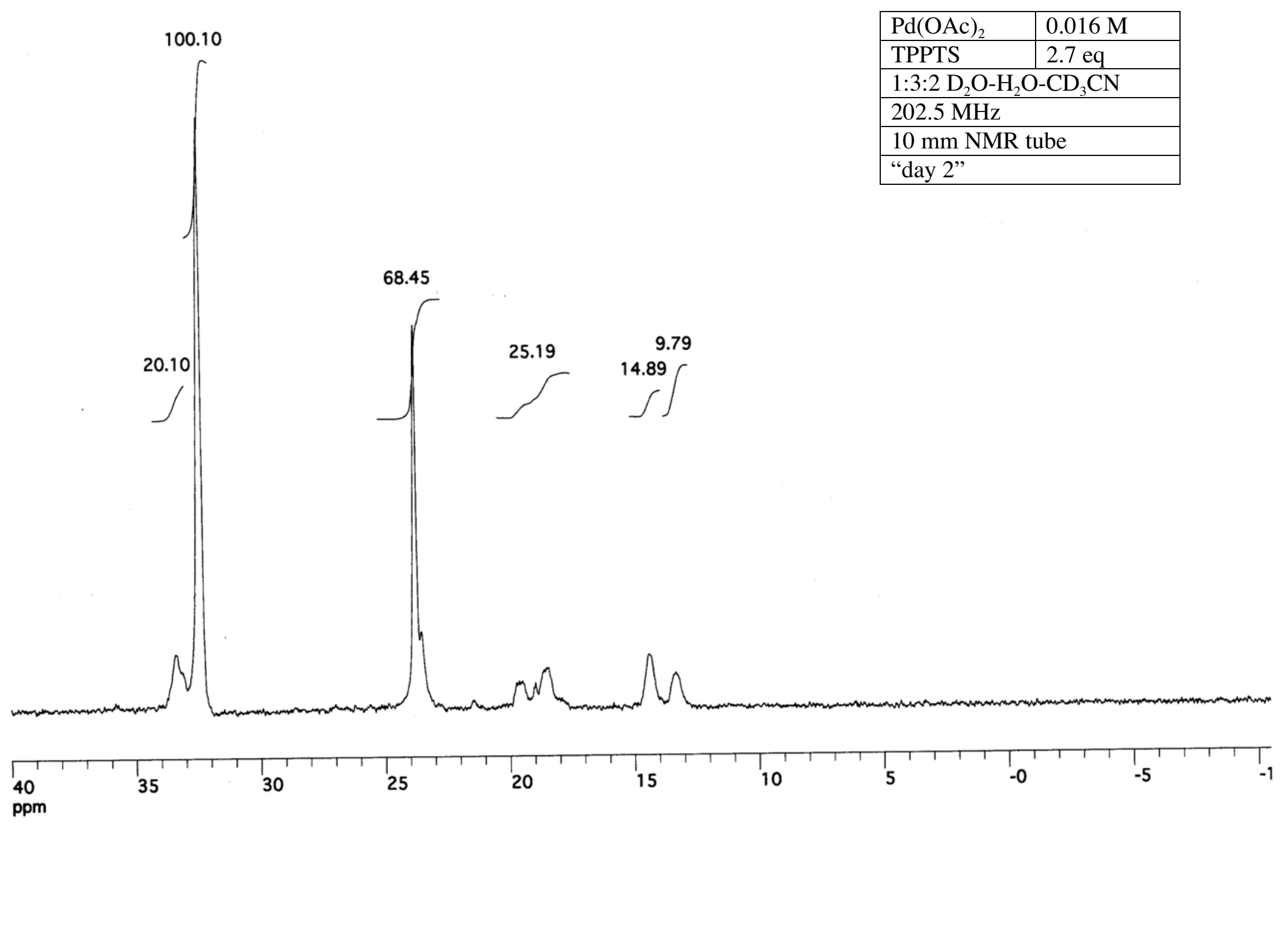




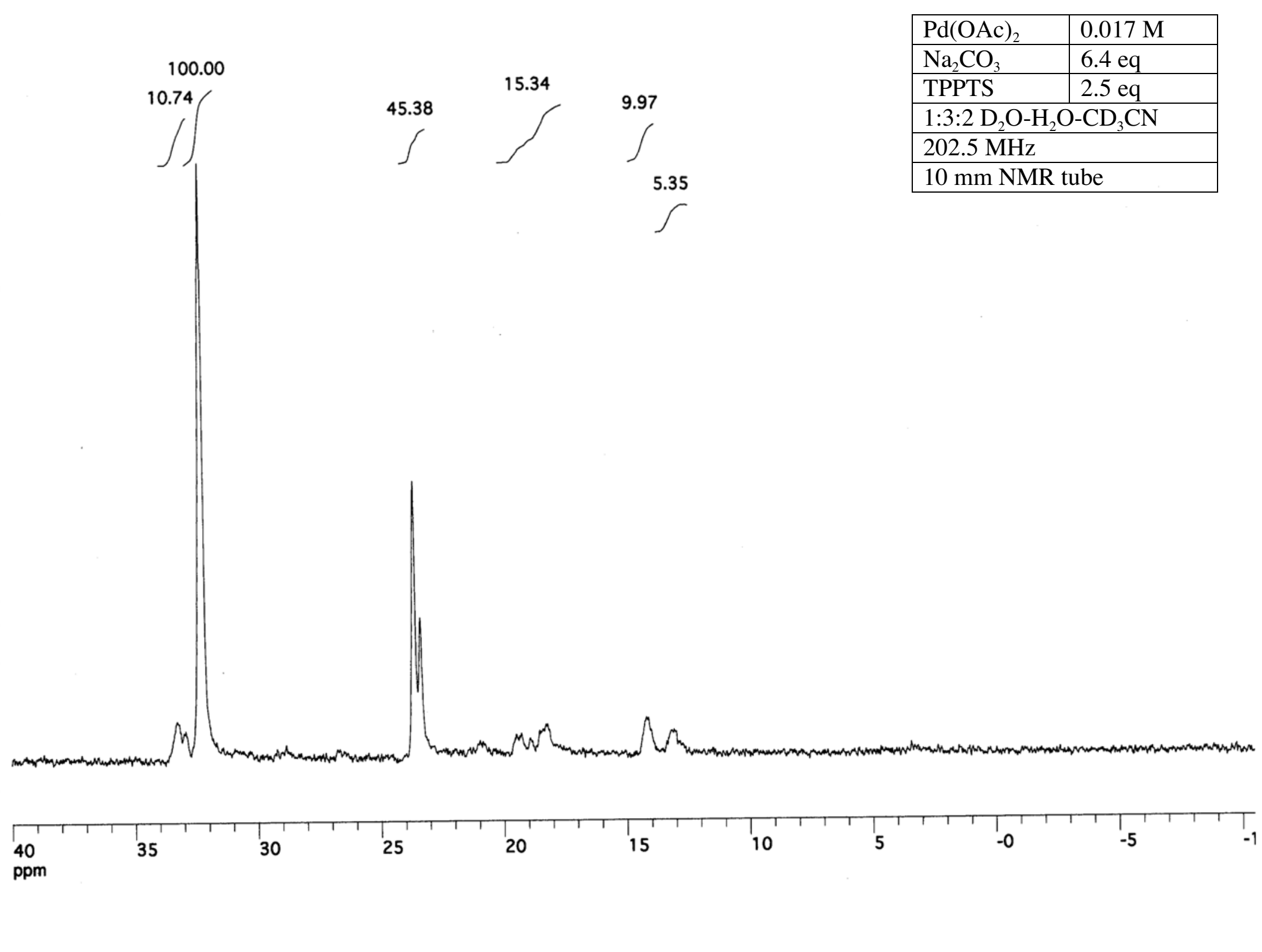




\begin{tabular}{|l|l|}
\hline $\mathrm{Pd}(\mathrm{OAc})_{2}$ & $0.017 \mathrm{M}$ \\
\hline $\mathrm{Na}_{2} \mathrm{CO}_{3}$ & $6.2 \mathrm{eq}$ \\
\hline TPPTS & $2.5 \mathrm{eq}$ \\
\hline $\mathrm{dA}$ & $2.0 \mathrm{eq}$ \\
\hline $1: 3: 2 \mathrm{D}_{2} \mathrm{O}-\mathrm{H}_{2} \mathrm{O}-\mathrm{CD}_{3} \mathrm{CN}$ \\
\hline $202.5 \mathrm{MHz}$ \\
\hline $10 \mathrm{~mm} \mathrm{NMR}$ tube \\
\hline
\end{tabular}

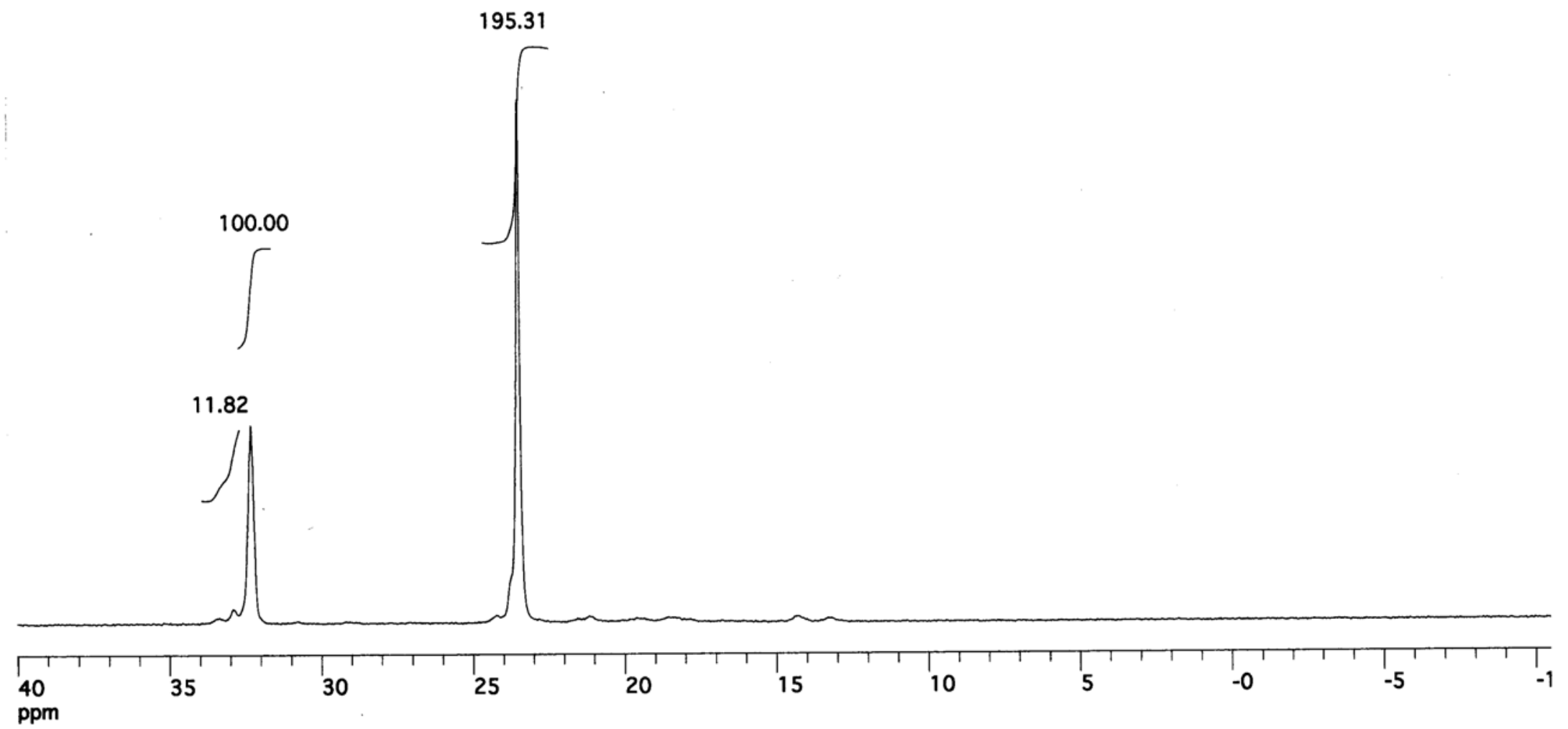




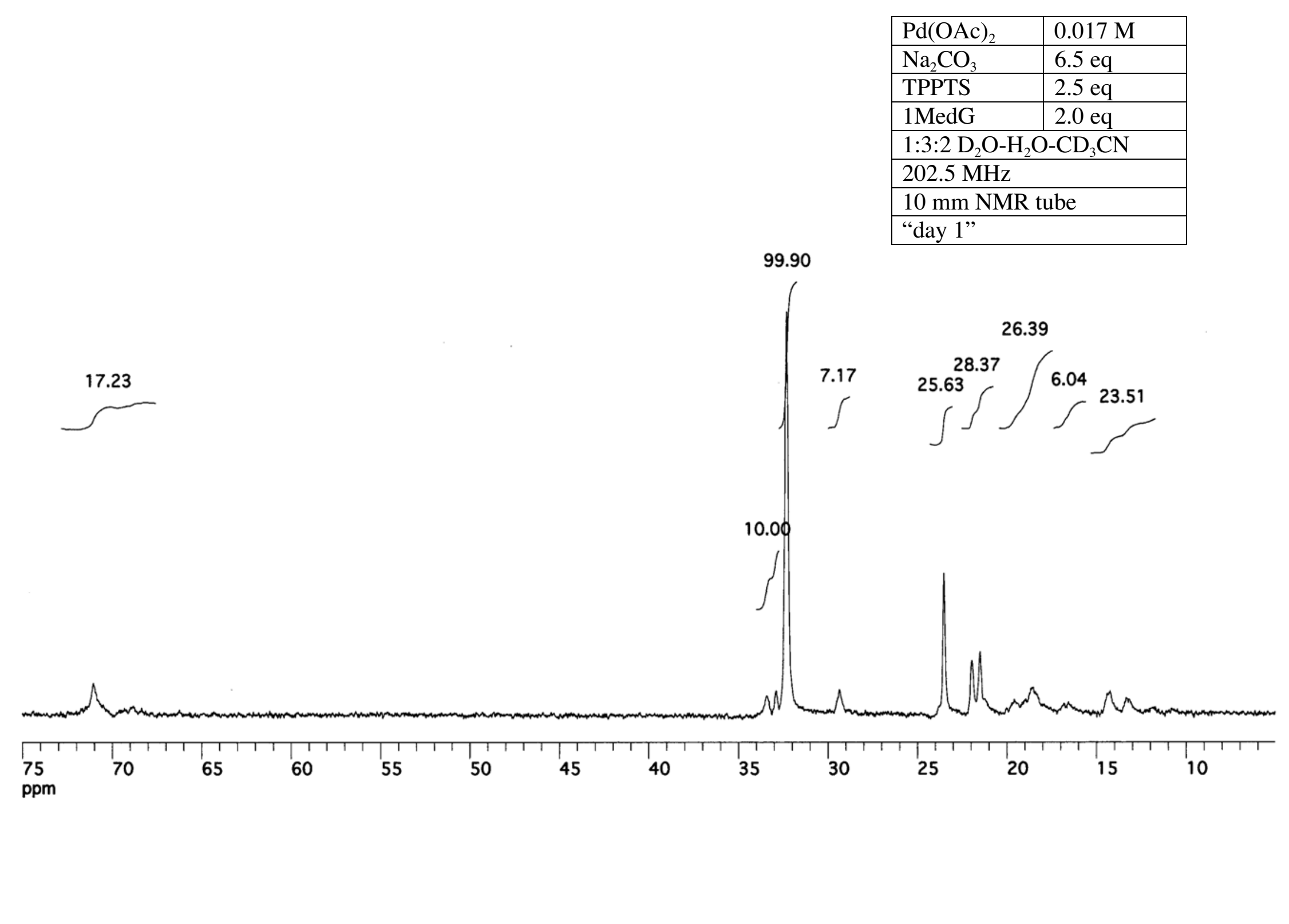




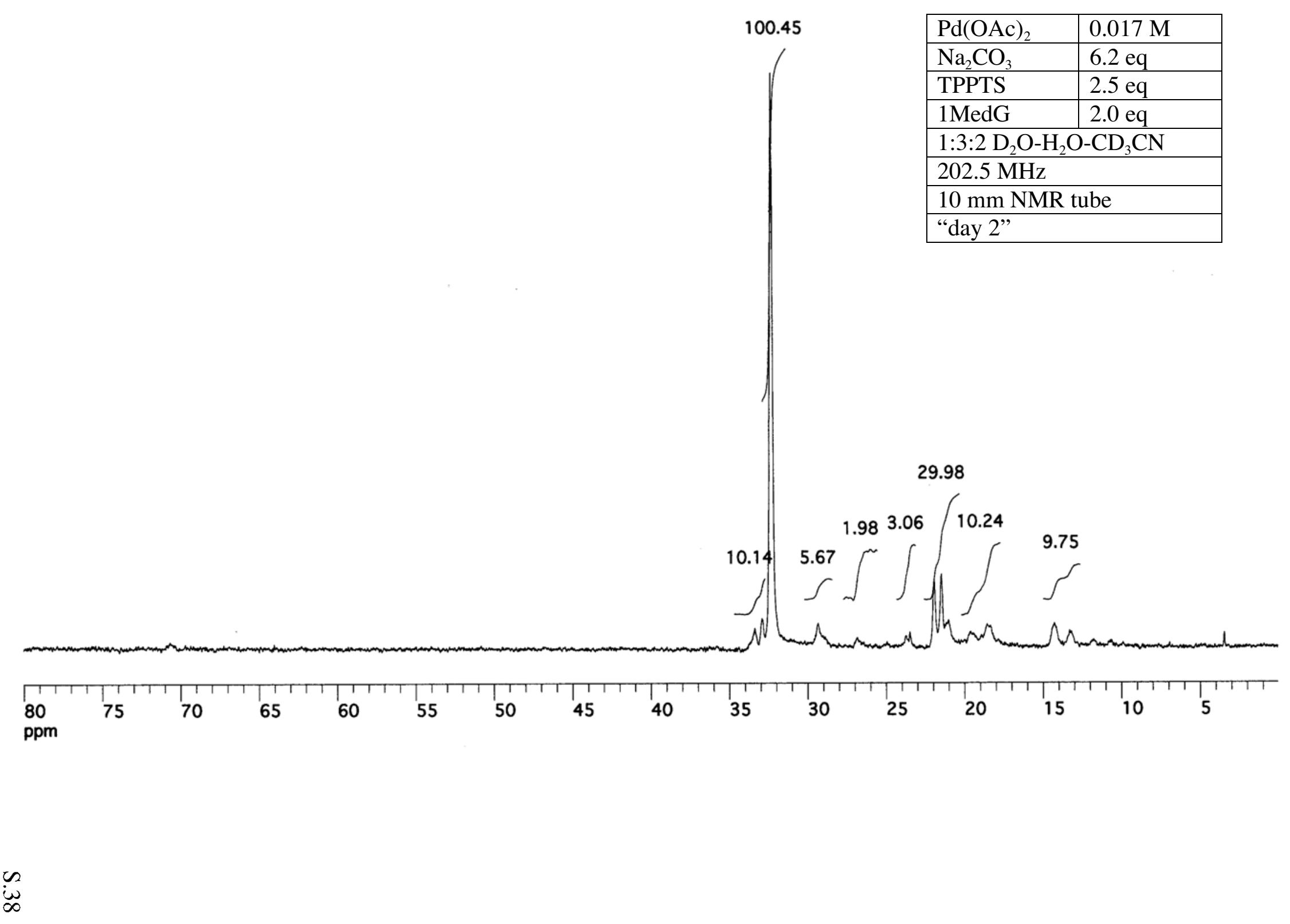




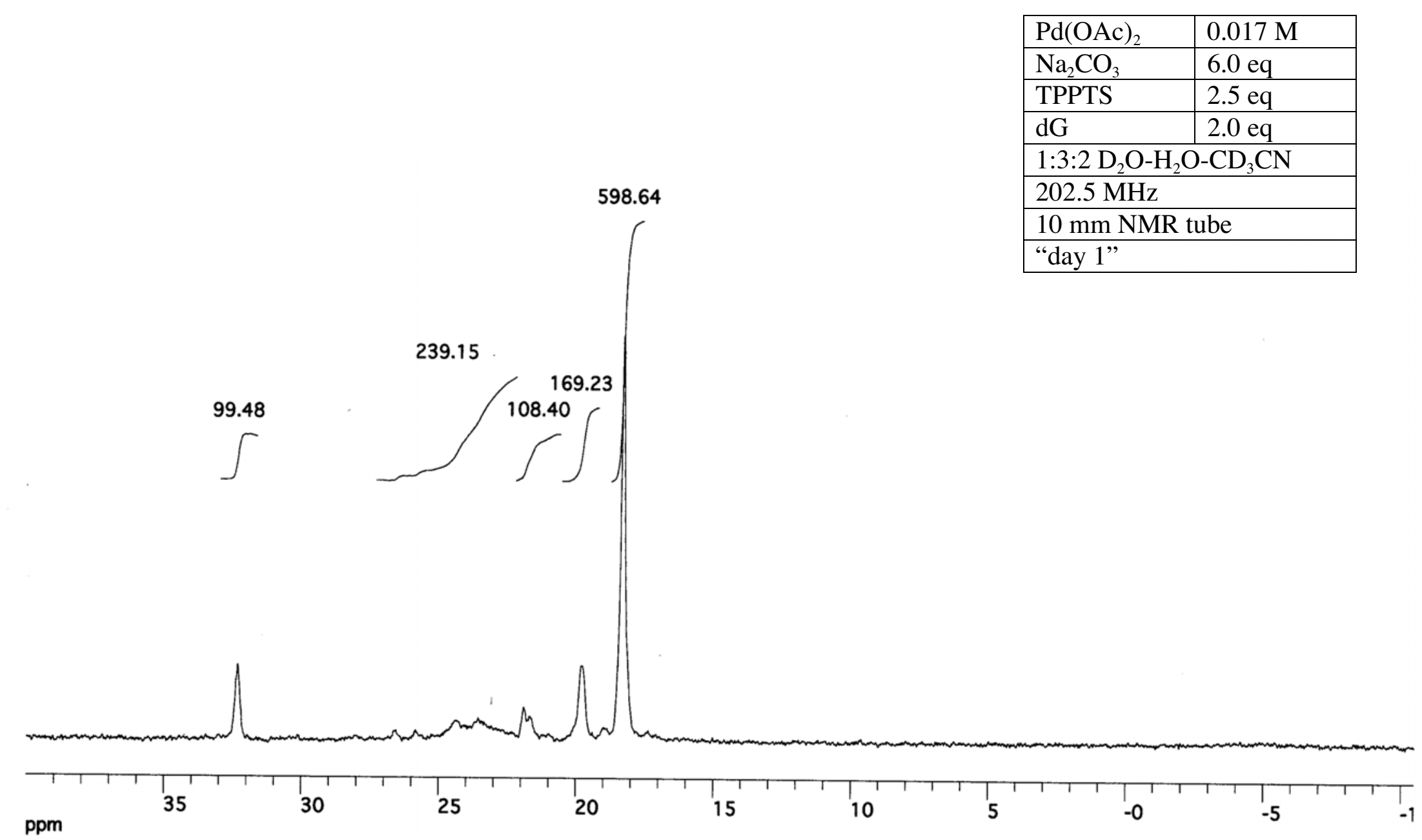




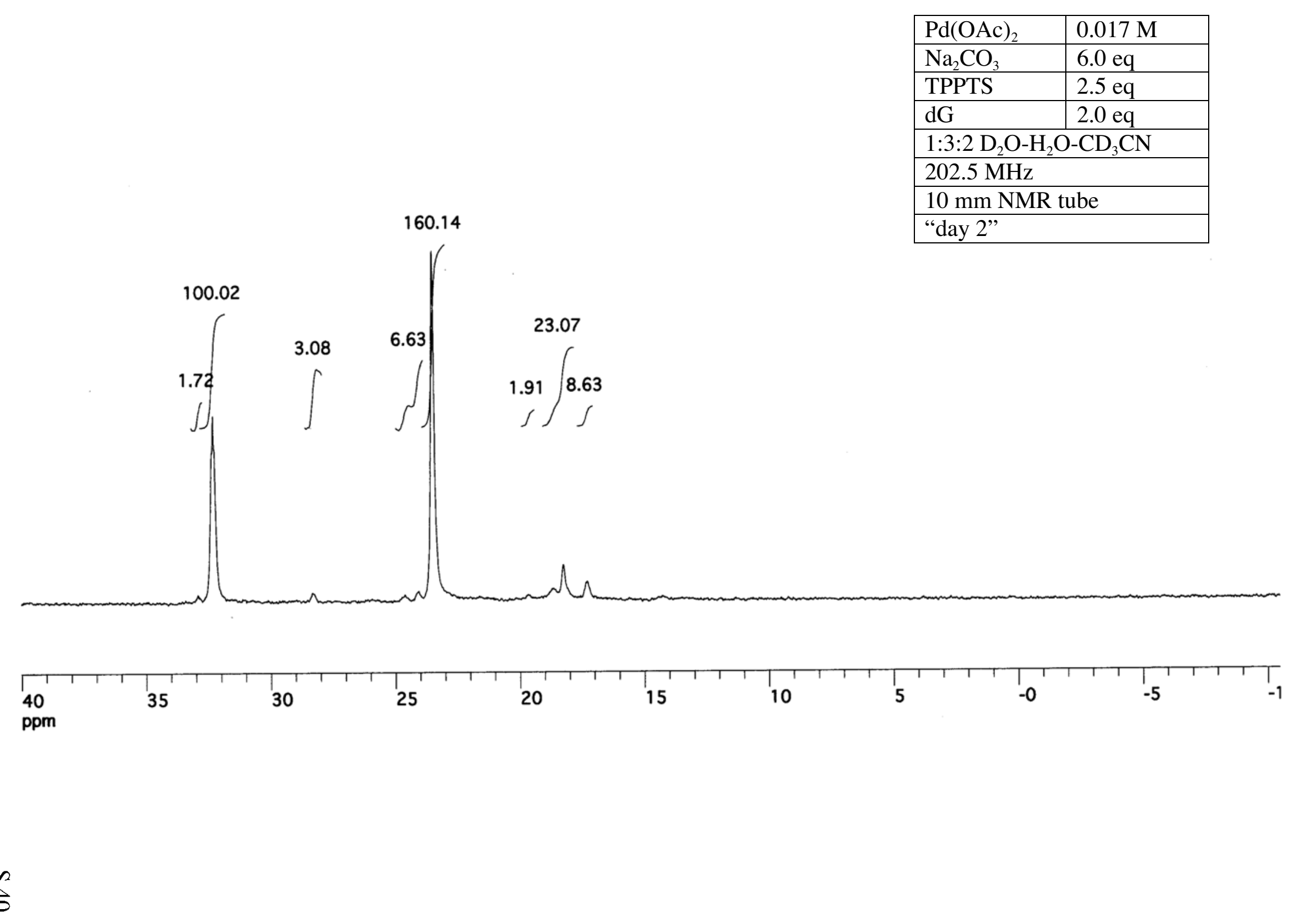




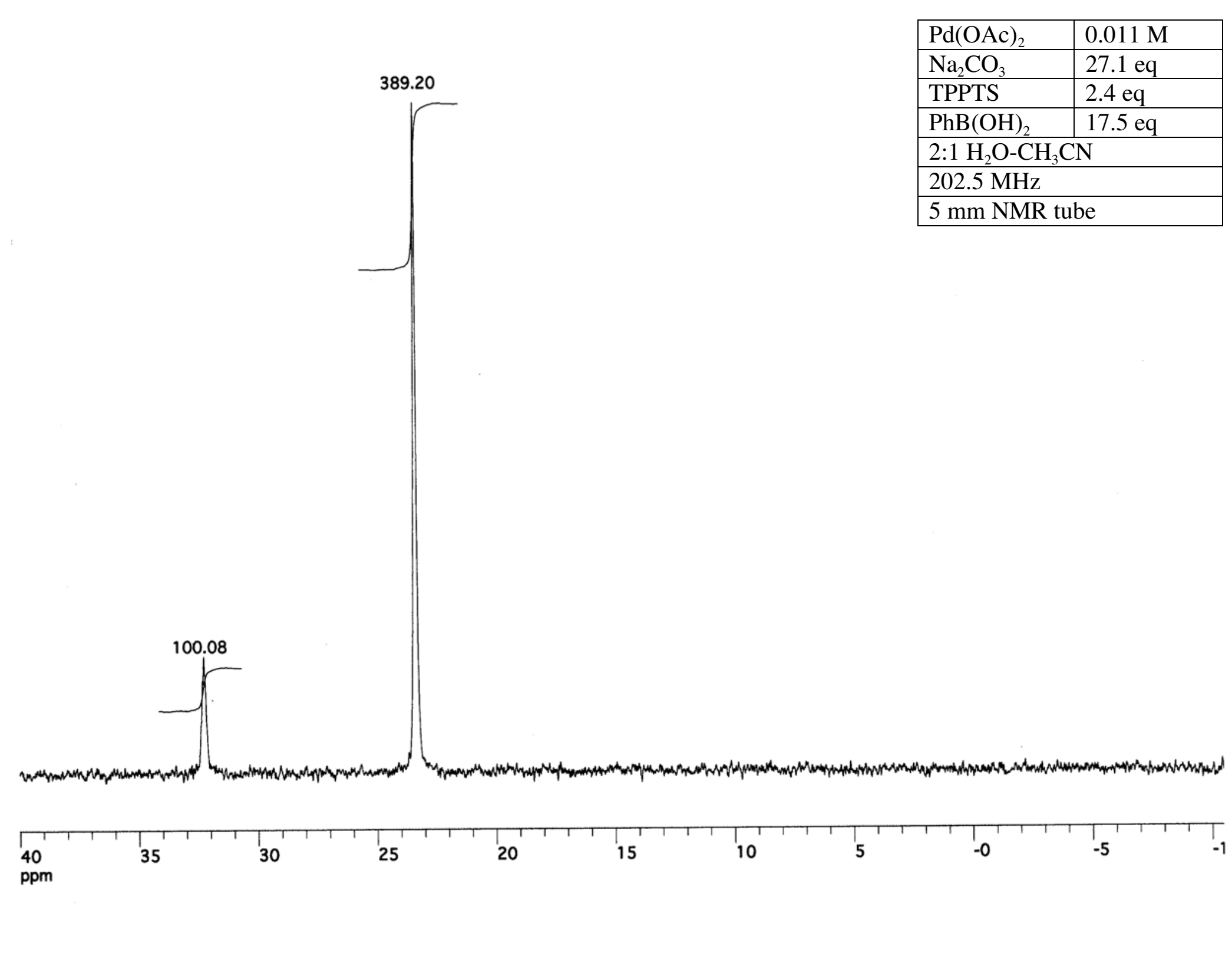




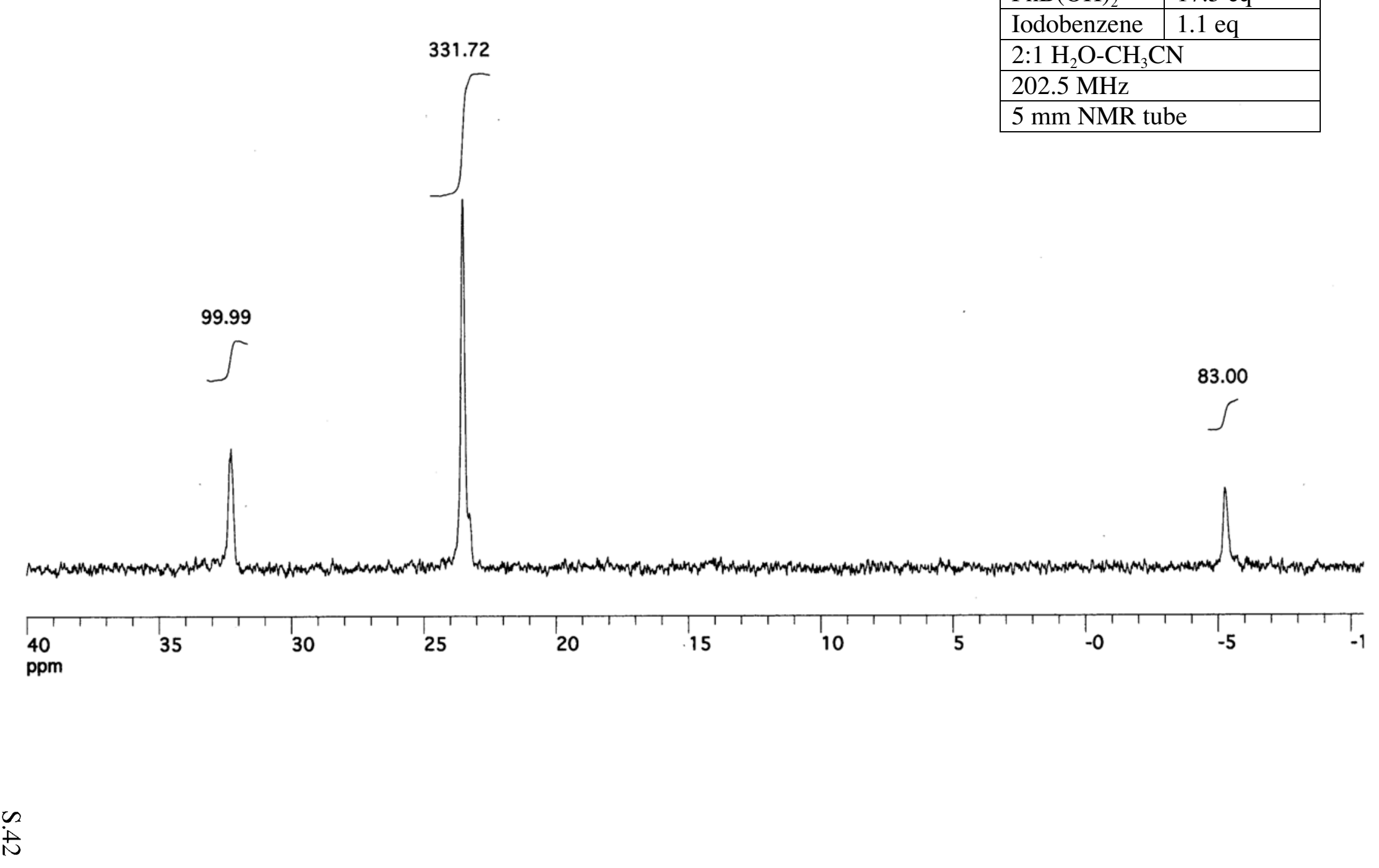

\begin{tabular}{|l|l|}
\hline $\mathrm{Pd}(\mathrm{OAc})_{2}$ & $0.011 \mathrm{M}$ \\
\hline $\mathrm{Na}_{2} \mathrm{CO}_{3}$ & $27.1 \mathrm{eq}$ \\
\hline $\mathrm{TPPTS}$ & $2.4 \mathrm{eq}$ \\
\hline $\mathrm{PhB}(\mathrm{OH})_{2}$ & $17.5 \mathrm{eq}$ \\
\hline Iodobenzene & $1.1 \mathrm{eq}$ \\
\hline $2: 1 \mathrm{H}_{2} \mathrm{O}-\mathrm{CH}_{3} \mathrm{CN}$ \\
\hline $202.5 \mathrm{MHz}$ \\
\hline $5 \mathrm{~mm} \mathrm{NMR}$ tube \\
\hline
\end{tabular}



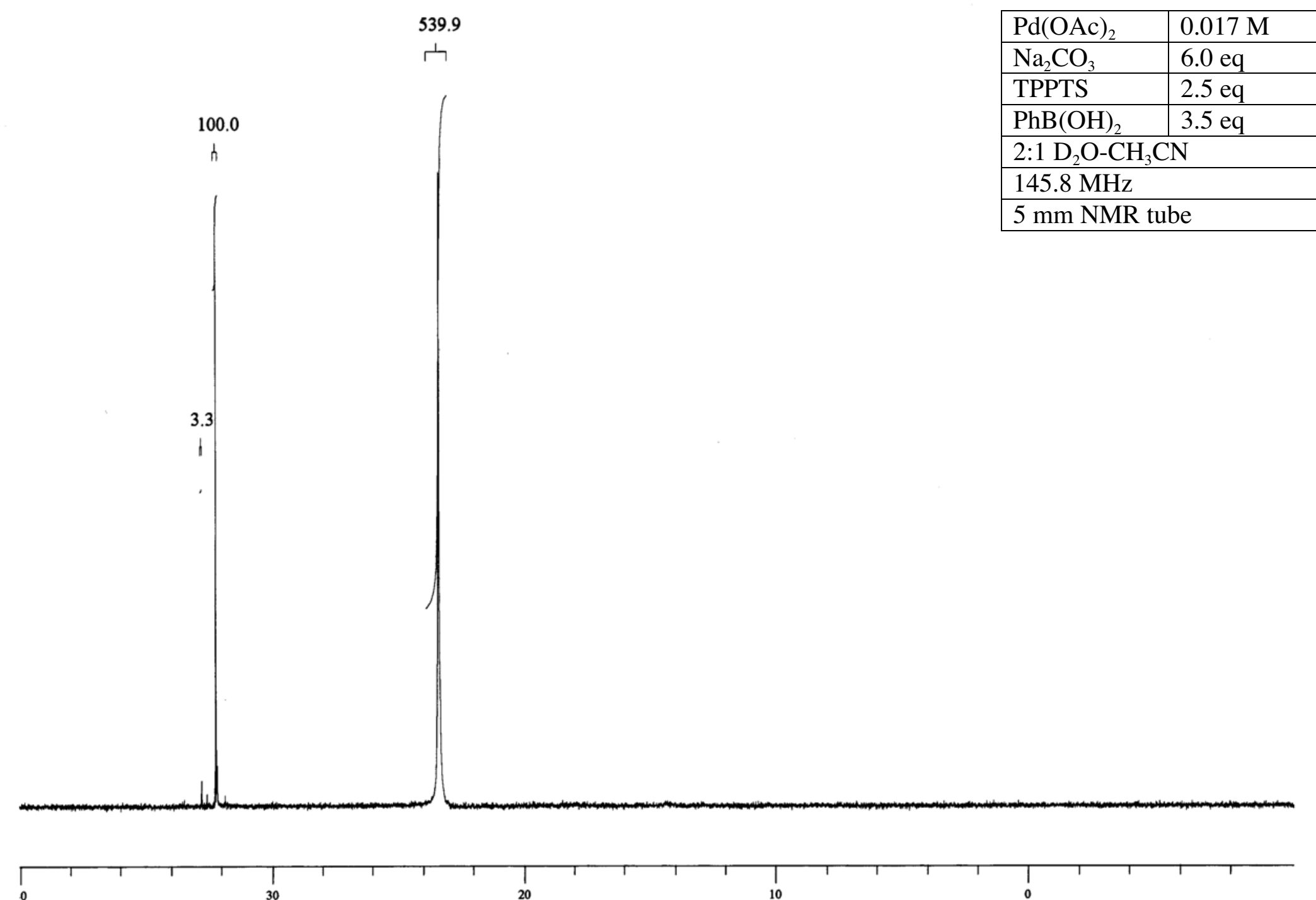


\begin{tabular}{|l|l|}
\hline $\mathrm{Pd}(\mathrm{OAc})_{2}$ & $0.017 \mathrm{M}$ \\
\hline $\mathrm{Na}_{2} \mathrm{CO}_{3}$ & $6.4 \mathrm{eq}$ \\
\hline $\mathrm{TPPTS}$ & $2.5 \mathrm{eq}$ \\
\hline $\mathrm{PhB}(\mathrm{OH})_{2}$ & $3.0 \mathrm{eq}$ \\
\hline $\mathrm{dA}$ & $2.8 \mathrm{eq}$ \\
\hline $1: 3: 2 \mathrm{D}_{2} \mathrm{O}-\mathrm{H}_{2} \mathrm{O}-\mathrm{CD}_{3} \mathrm{CN}$ \\
\hline $202.5 \mathrm{MHz}$ \\
\hline $10 \mathrm{~mm} \mathrm{NMR}$ tube \\
\hline
\end{tabular}

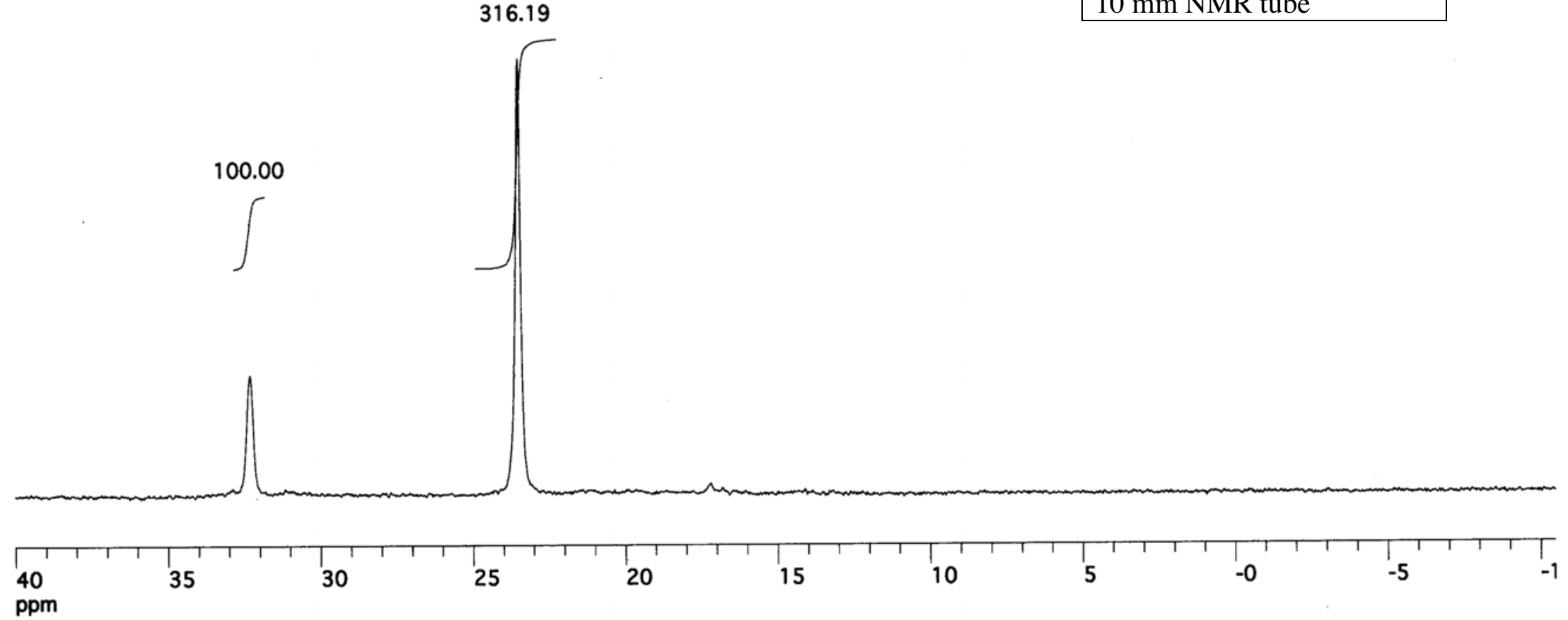




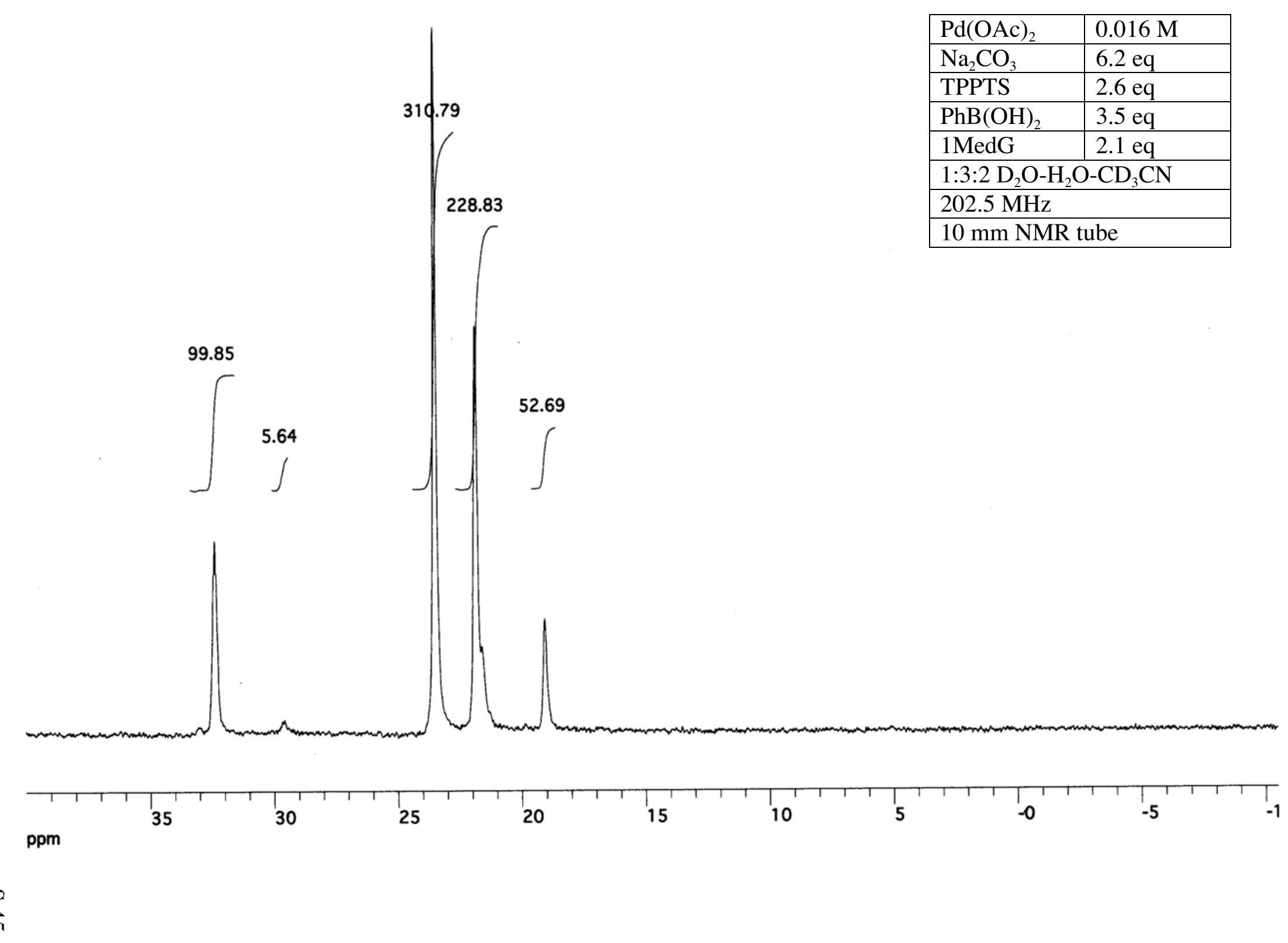




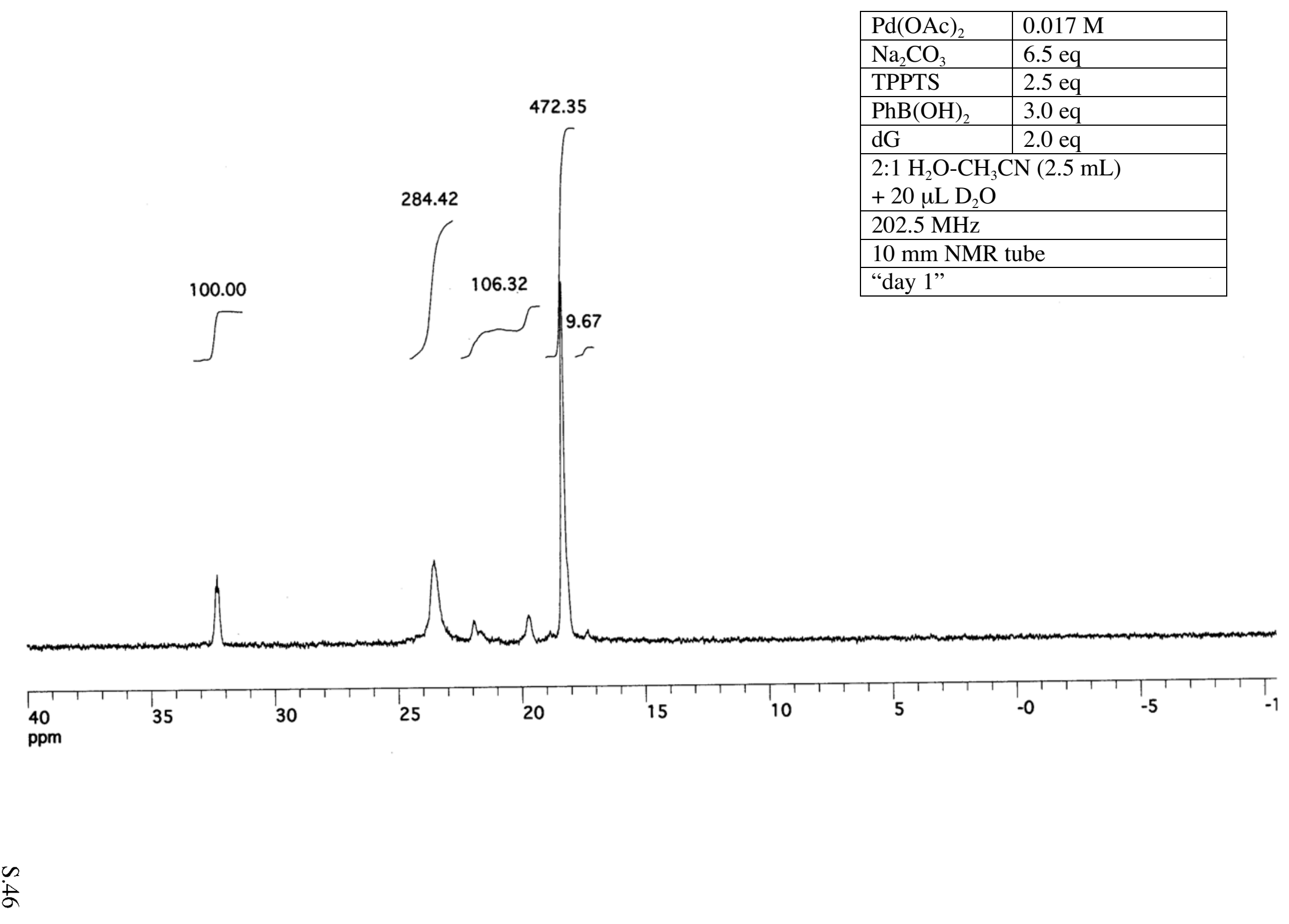




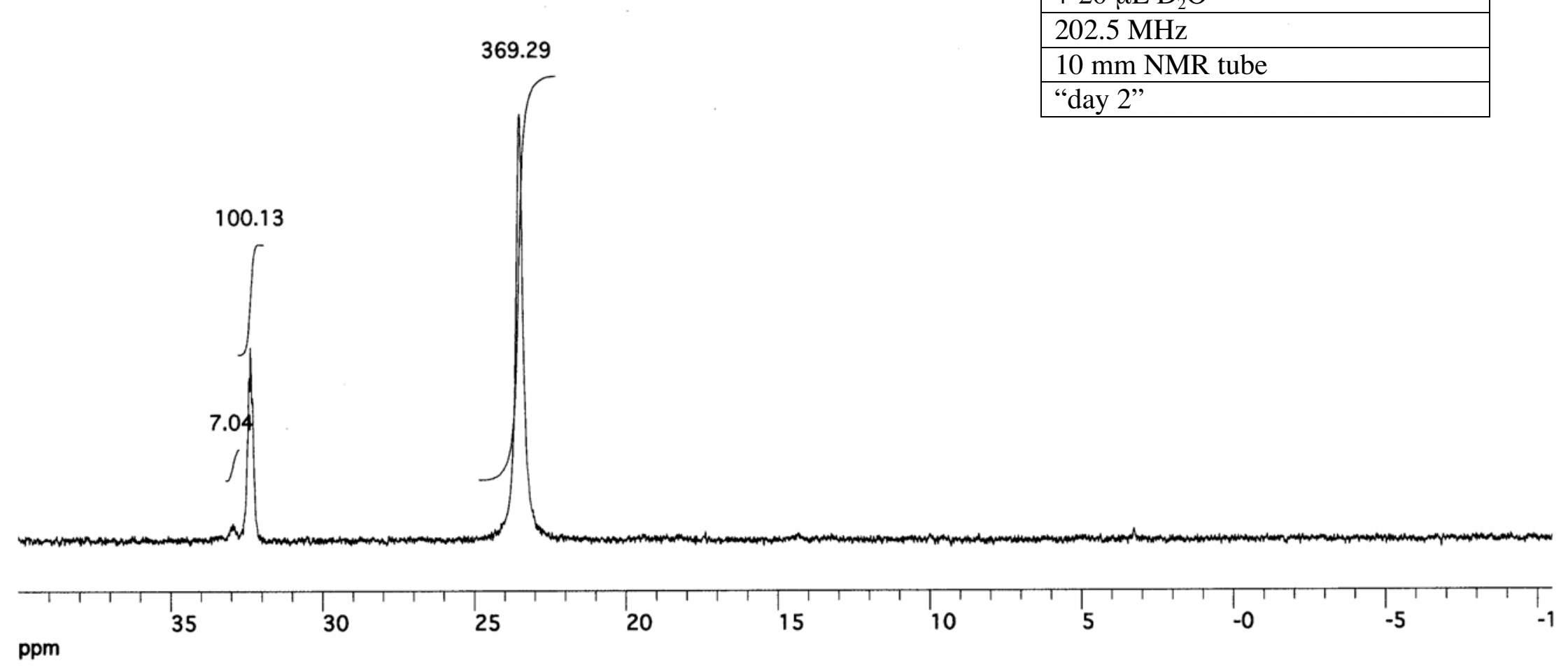




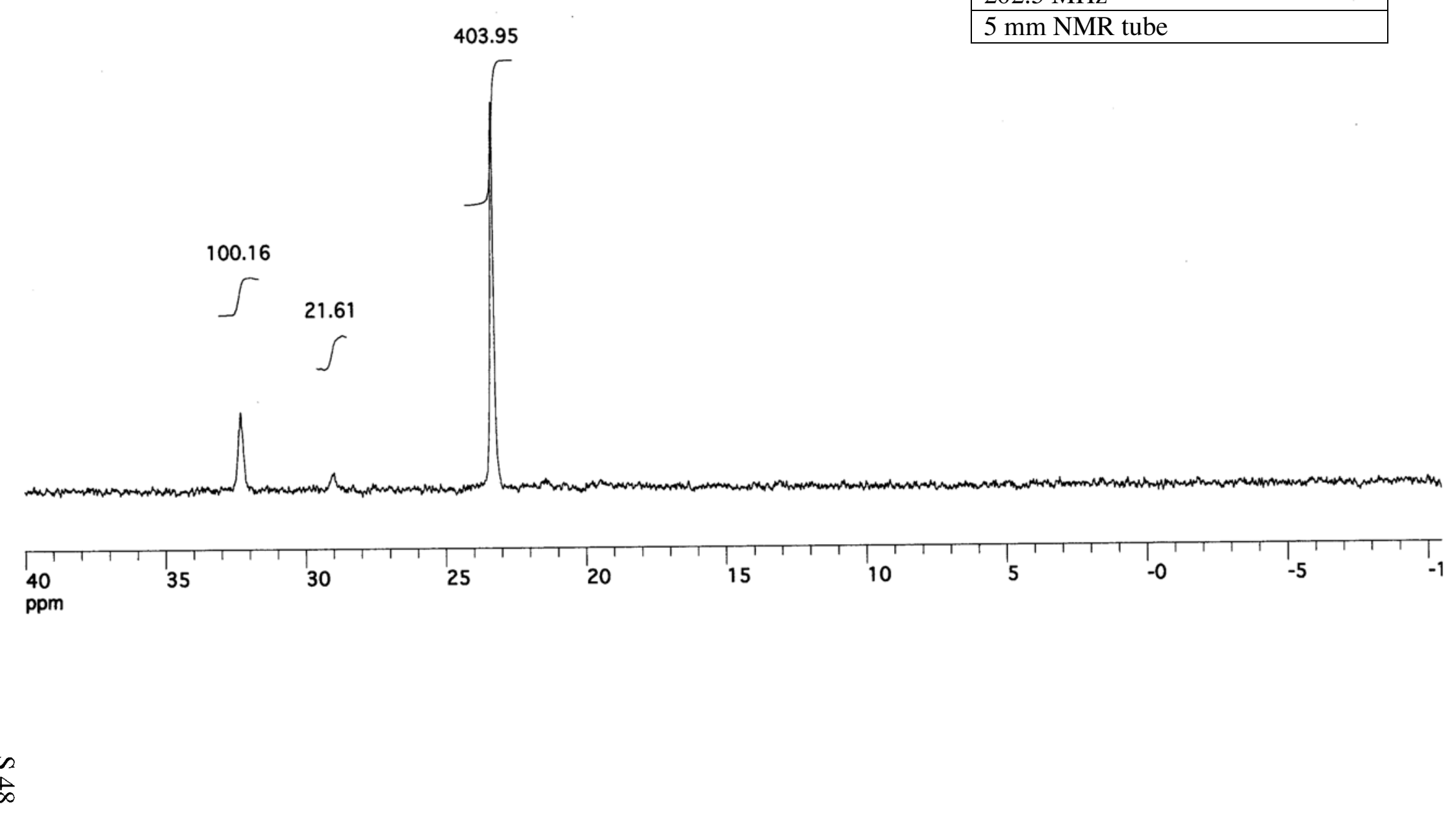

\begin{tabular}{|l|l|}
\hline $\mathrm{Pd}(\mathrm{OAc})_{2}$ & $0.01 \mathrm{M}$ \\
\hline $\mathrm{Na}_{2} \mathrm{CO}_{3}$ & $35.8 \mathrm{eq}$ \\
\hline $\mathrm{TPPTS}$ & $2.5 \mathrm{eq}$ \\
\hline $\mathrm{PhB}(\mathrm{OH})_{2}$ & $18.6 \mathrm{eq}$ \\
\hline $\mathrm{dG}$ & $1.0 \mathrm{eq}$ \\
\hline $2: 1 \mathrm{D}_{2} \mathrm{O}-\mathrm{CD}_{3} \mathrm{CN}$ \\
\hline $202.5 \mathrm{MHz}$ \\
\hline $5 \mathrm{~mm} \mathrm{NMR} \mathrm{tube}$ \\
\hline
\end{tabular}


181.39

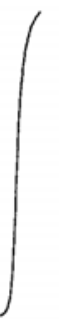

\begin{tabular}{|l|l|}
\hline $\mathrm{Pd}(\mathrm{OAc})_{2}$ & $0.01 \mathrm{M}$ \\
\hline $\mathrm{Na}_{2} \mathrm{CO}_{3}$ & $35.8 \mathrm{eq}$ \\
\hline $\mathrm{TPPTS}$ & $2.5 \mathrm{eq}$ \\
\hline $\mathrm{PhB}(\mathrm{OH})_{2}$ & $18.6 \mathrm{eq}$ \\
\hline $\mathrm{dG}$ & $1.0 \mathrm{eq}$ \\
\hline Iodobenzene & ca. $360 \mathrm{eq}$ \\
\hline 2:1 $\mathrm{D}_{2} \mathrm{O}-\mathrm{CD}_{3} \mathrm{CN}$ \\
\hline $202.5 \mathrm{MHz}$ \\
\hline $5 \mathrm{~mm} \mathrm{NMR} \mathrm{tube}$ \\
\hline
\end{tabular}
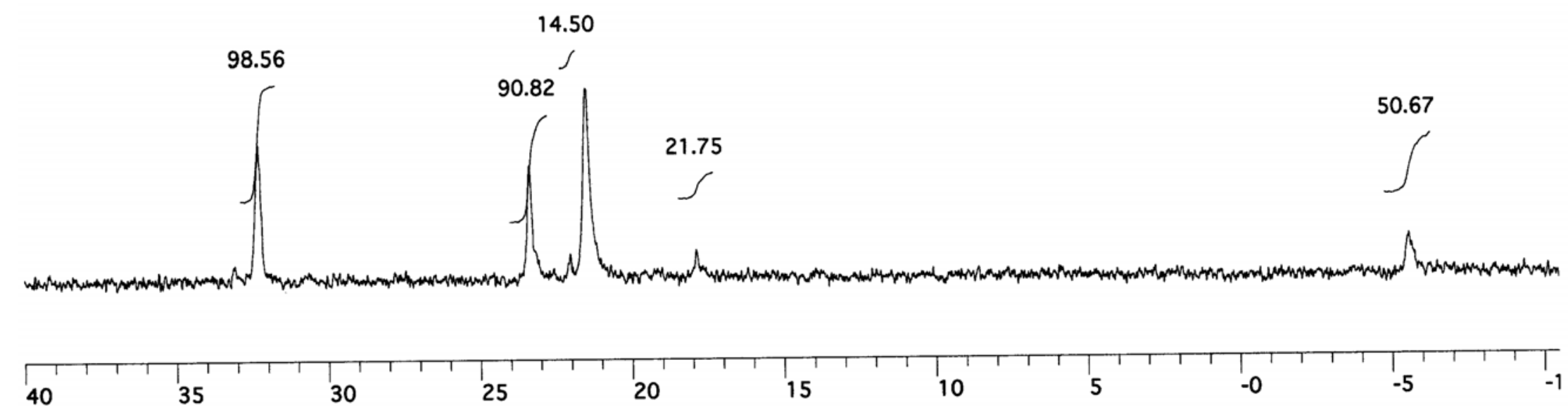

ppm

20

15

10

(1)




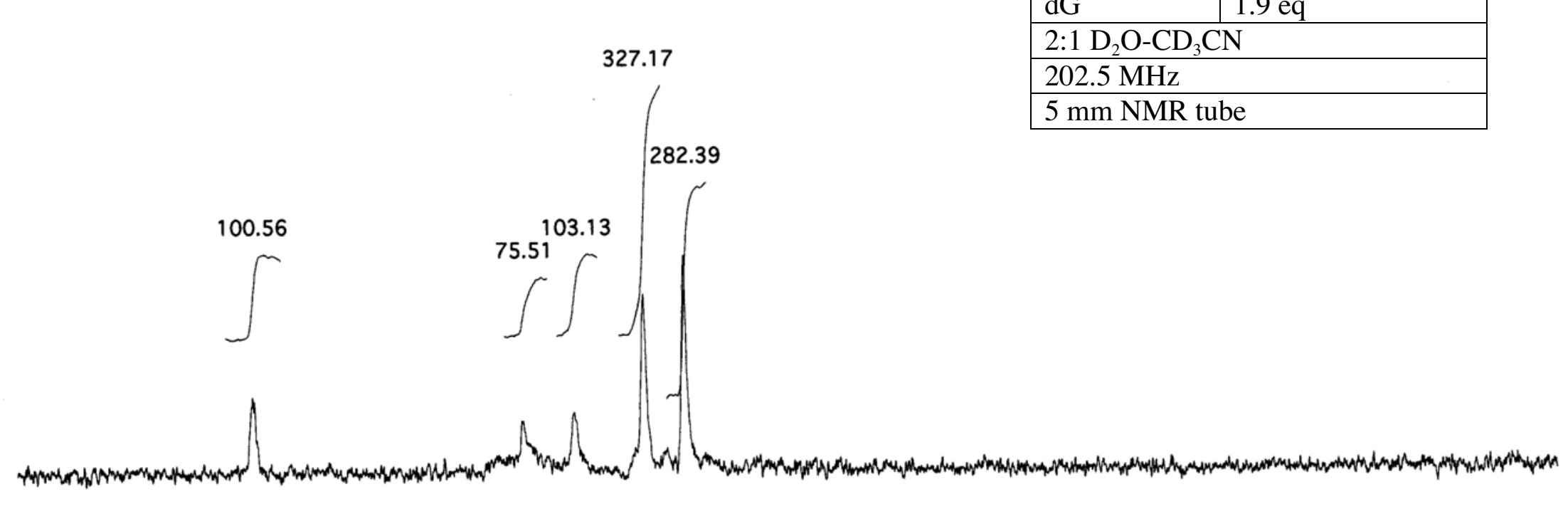

\begin{tabular}{|l|l|}
\hline $\mathrm{Pd}(\mathrm{OAc})_{2}$ & $0.01 \mathrm{M}$ \\
\hline $\mathrm{Na}_{2} \mathrm{CO}_{3}$ & $33.2 \mathrm{eq}$ \\
\hline $\mathrm{TPPTS}$ & $2.6 \mathrm{eq}$ \\
\hline $\mathrm{PhB}(\mathrm{OH})_{2}$ & $14.6 \mathrm{eq}$ \\
\hline $\mathrm{dG}$ & $1.9 \mathrm{eq}$ \\
\hline $2: 1 \mathrm{D}_{2} \mathrm{O}-\mathrm{CD}_{3} \mathrm{CN}$ \\
\hline $202.5 \mathrm{MHz}$ \\
\hline $5 \mathrm{~mm} \mathrm{NMR} \mathrm{tube}$ \\
\hline
\end{tabular}

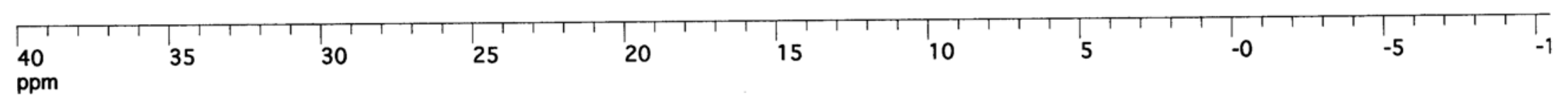




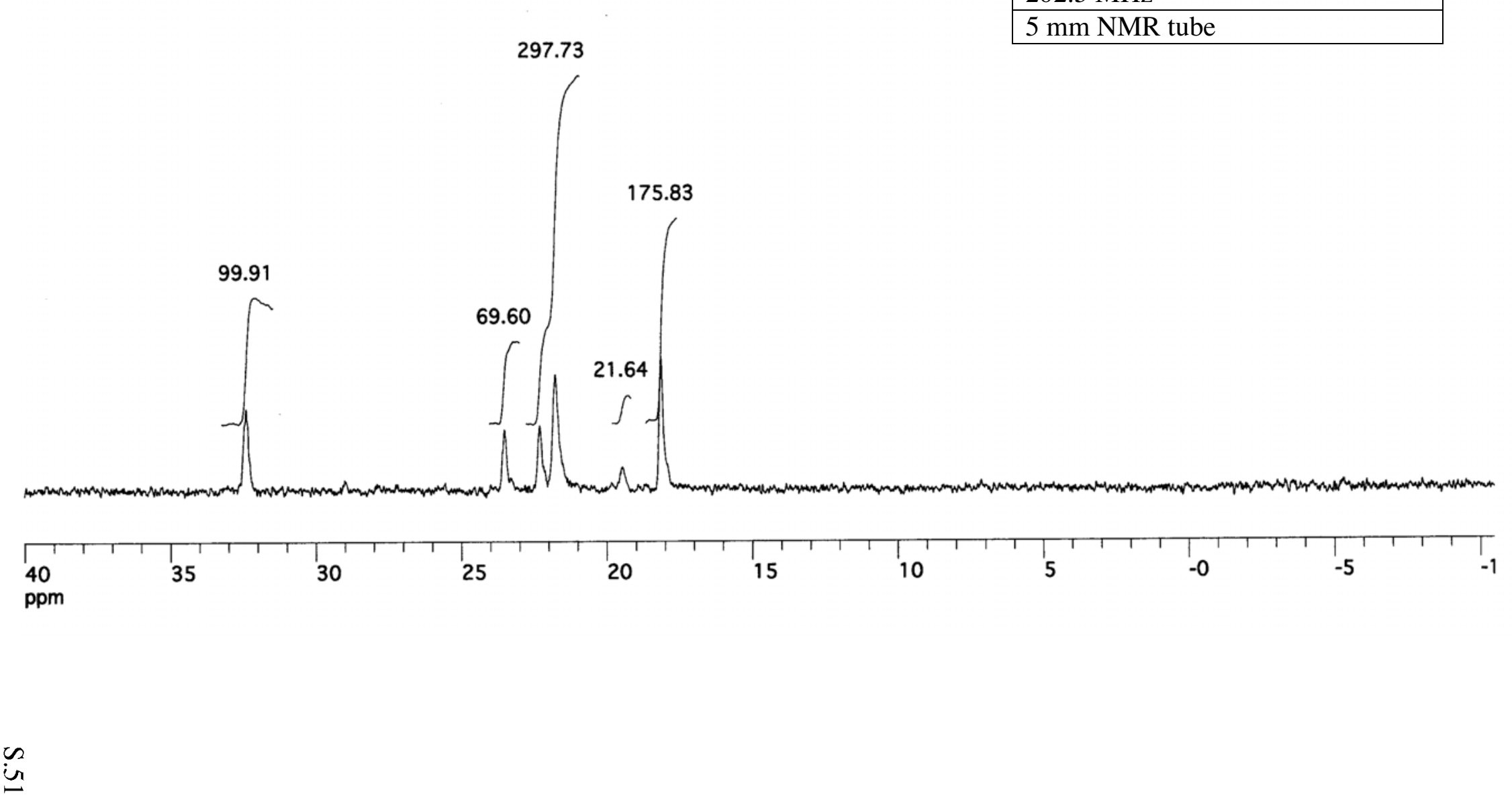

\begin{tabular}{|l|l|}
\hline $\mathrm{Pd}(\mathrm{OAc})_{2}$ & $0.01 \mathrm{M}$ \\
\hline $\mathrm{Na}_{2} \mathrm{CO}_{3}$ & $33.2 \mathrm{eq}$ \\
\hline $\mathrm{TPPTS}$ & $2.6 \mathrm{eq}$ \\
\hline $\mathrm{PhB}(\mathrm{OH})_{2}$ & $14.6 \mathrm{eq}$ \\
\hline $\mathrm{dG}$ & $1.9 \mathrm{eq}$ \\
\hline Iodobezene & $8.1 \mathrm{eq}$ \\
\hline $2: 1 \mathrm{D}_{2} \mathrm{O}-\mathrm{CD}_{3} \mathrm{CN}$ \\
\hline $202.5 \mathrm{MHz}$ \\
\hline $5 \mathrm{~mm} \mathrm{NMR} \mathrm{tube}$ \\
\hline
\end{tabular}




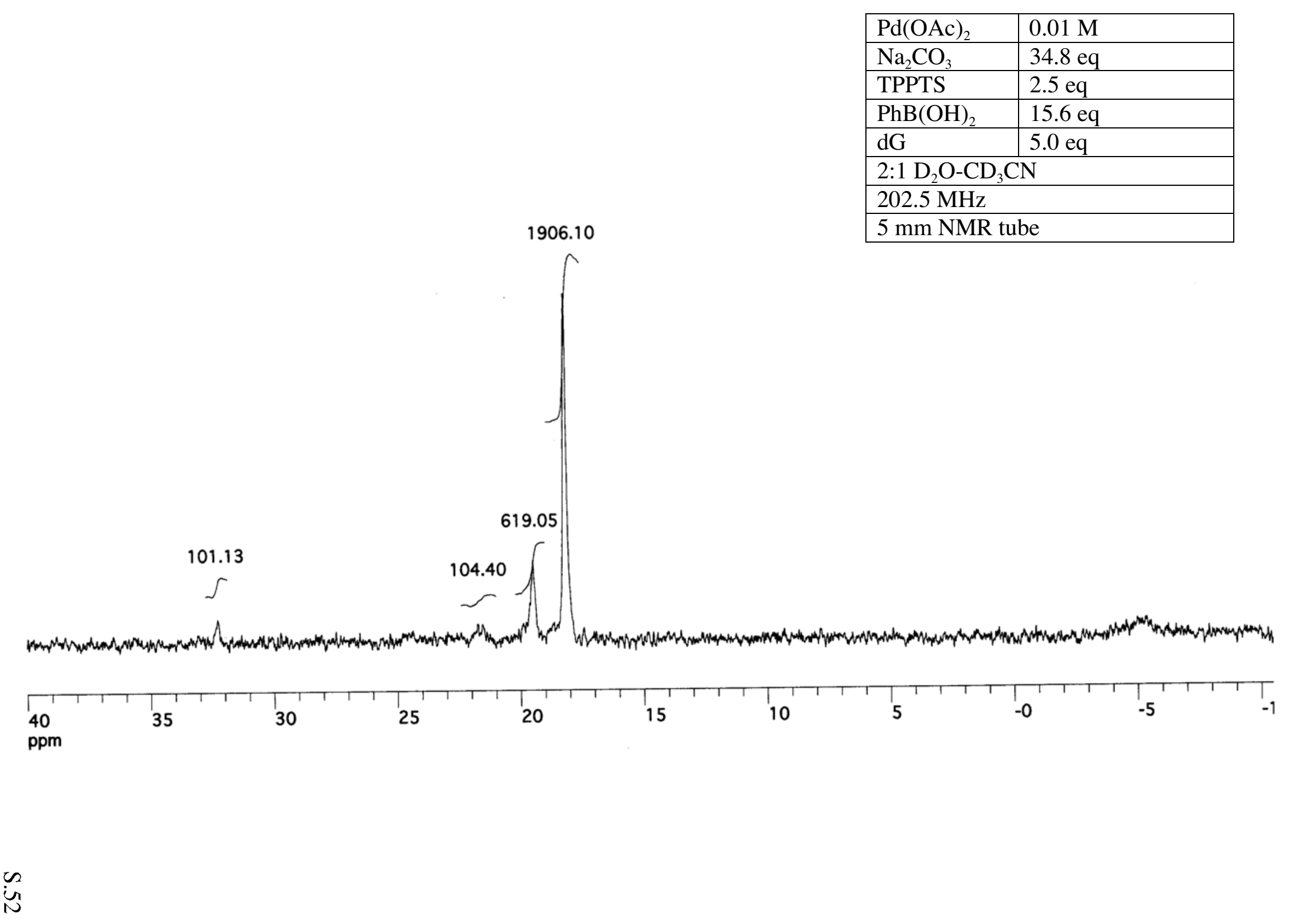




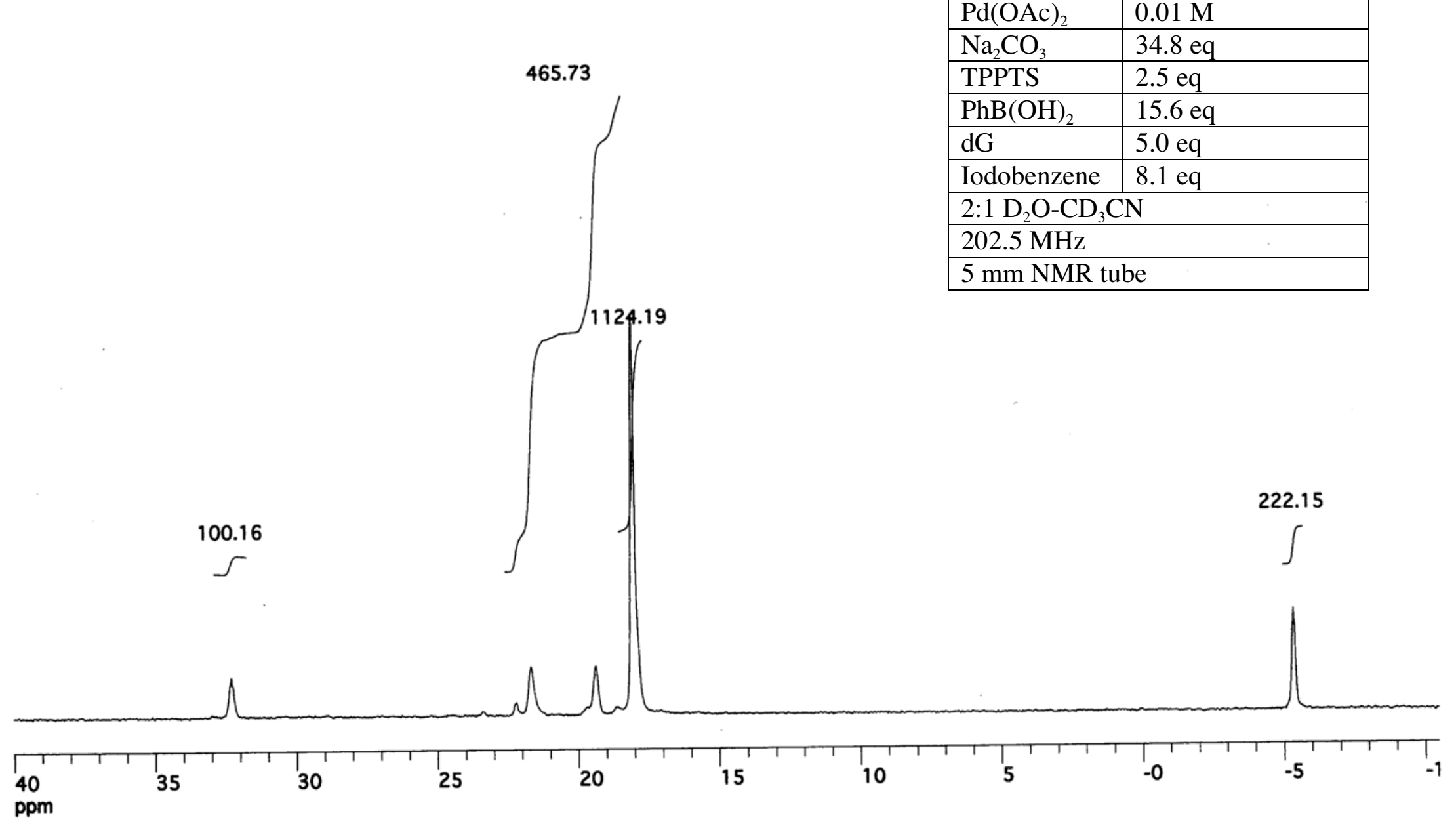




\begin{tabular}{|l|l|}
\hline $\mathrm{Pd}(\mathrm{OAc})_{2}$ & $0.005 \mathrm{M}$ \\
\hline $\mathrm{Na}_{2} \mathrm{CO}_{3}$ & $6.0 \mathrm{eq}$ \\
\hline TXPTS & $2.6 \mathrm{eq}$ \\
\hline $2: 1 \mathrm{D}_{2} \mathrm{O}-\mathrm{CD}_{3} \mathrm{CN}$ \\
\hline $202.5 \mathrm{MHz}$ \\
\hline $5 \mathrm{~mm} \mathrm{NMR}$ tube \\
\hline "day 1" \\
\hline
\end{tabular}

523.91

25.34

1

256.21

99.97
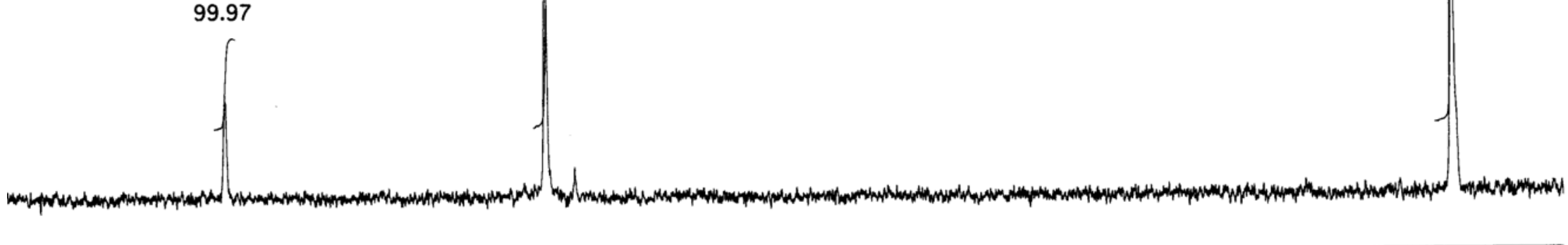

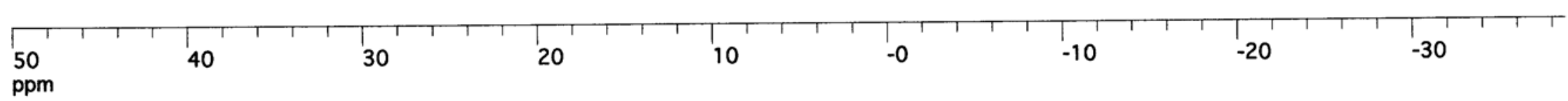

in 


\begin{tabular}{|l|l|}
\hline $\mathrm{Pd}(\mathrm{OAc})_{2}$ & $0.005 \mathrm{M}$ \\
\hline $\mathrm{Na}_{2} \mathrm{CO}_{3}$ & $6.0 \mathrm{eq}$ \\
\hline TXPTS & $2.6 \mathrm{eq}$ \\
\hline $2: 1 \mathrm{D}_{2} \mathrm{O}-\mathrm{CD}_{3} \mathrm{CN}$ \\
\hline $202.5 \mathrm{MHz}$ \\
\hline $5 \mathrm{~mm} \mathrm{NMR}$ tube \\
\hline "day 2" \\
\hline
\end{tabular}

442.66

343.11

100.00
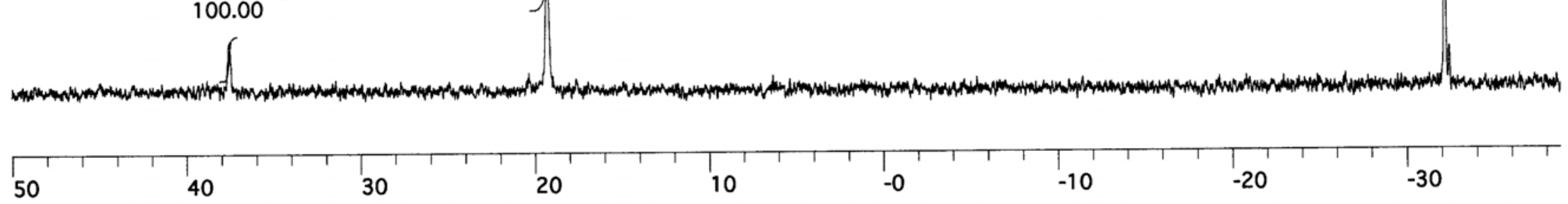

ppm

$-0$

$-10$

$-20$

$-30$ 


\begin{tabular}{|l|l|}
\hline $\mathrm{Pd}(\mathrm{OAc})_{2}$ & $0.005 \mathrm{M}$ \\
\hline $\mathrm{Na}_{2} \mathrm{CO}_{3}$ & $18.0 \mathrm{eq}$ \\
\hline TXPTS & $3.8 \mathrm{eq}$ \\
\hline $2: 1 \mathrm{D}_{2} \mathrm{O}-\mathrm{CD}_{3} \mathrm{CN}$ \\
\hline $202.5 \mathrm{MHz}$ \\
\hline $5 \mathrm{~mm} \mathrm{NMR} \mathrm{tube}$ \\
\hline "day 1" \\
\hline
\end{tabular}

42.03

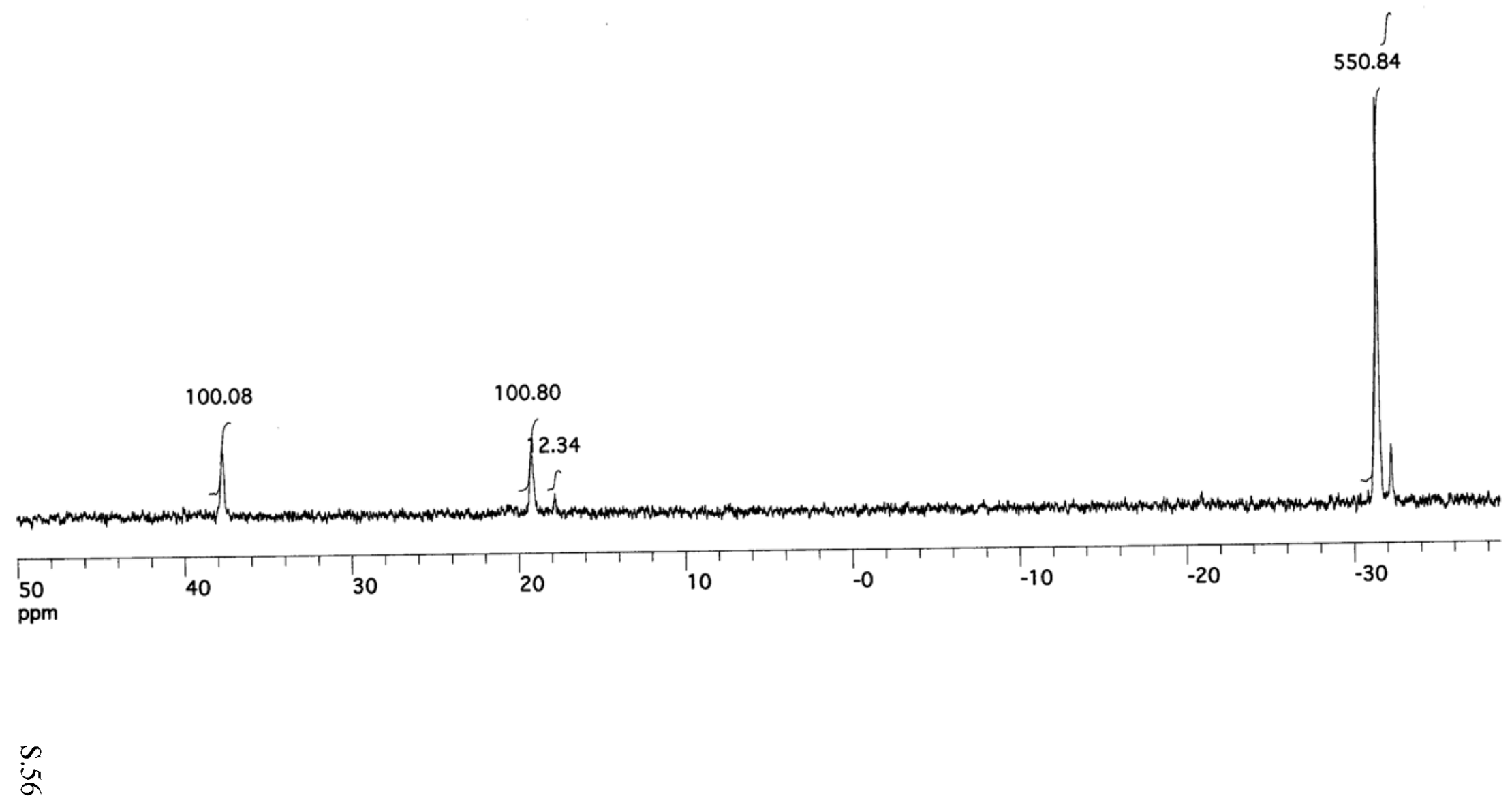




\begin{tabular}{|l|l|}
\hline $\mathrm{Pd}(\mathrm{OAc})_{2}$ & $0.005 \mathrm{M}$ \\
\hline $\mathrm{Na}_{2} \mathrm{CO}_{3}$ & $18.0 \mathrm{eq}$ \\
\hline TXPTS & $3.8 \mathrm{eq}$ \\
\hline $2: 1 \mathrm{D}_{2} \mathrm{O}-\mathrm{CD}_{3} \mathrm{CN}$ \\
\hline $202.5 \mathrm{MHz}$ \\
\hline $5 \mathrm{~mm} \mathrm{NMR} \mathrm{tube}$ \\
\hline "day 2" \\
\hline
\end{tabular}
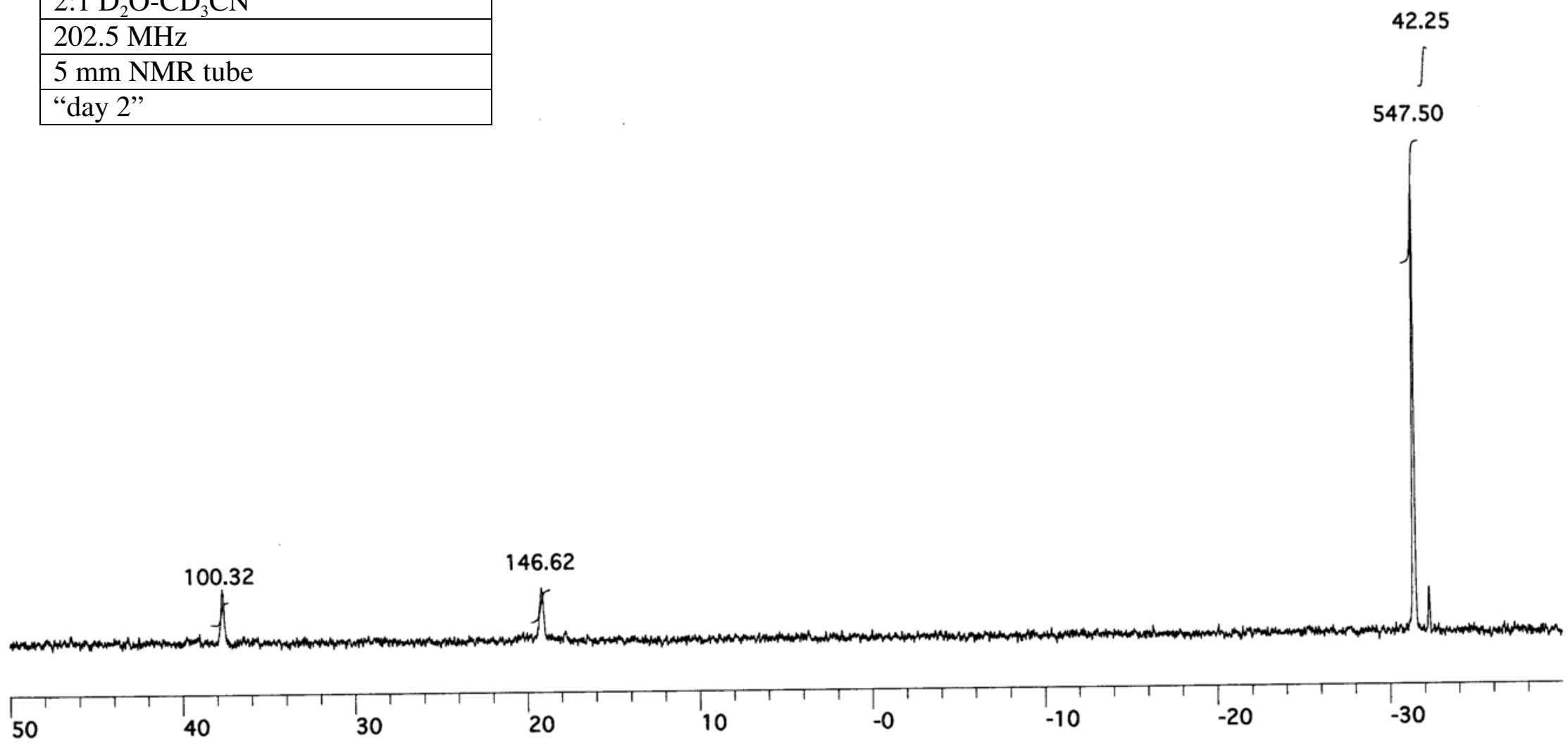

ppm

.

- 


\begin{tabular}{|l|l|}
\hline $\mathrm{Pd}(\mathrm{OAc})_{2}$ & $0.017 \mathrm{M}$ \\
\hline $\mathrm{Na}_{2} \mathrm{CO}_{3}$ & $7.3 \mathrm{eq}$ \\
\hline TXPTS & $2.5 \mathrm{eq}$ \\
\hline $\mathrm{dA}$ & $2.0 \mathrm{eq}$ \\
\hline $1: 3: 2 \mathrm{D}_{2} \mathrm{O}-\mathrm{H}_{2} \mathrm{O}-\mathrm{CD}_{3} \mathrm{CN}$ \\
\hline $202.5 \mathrm{MHz}$ \\
\hline $10 \mathrm{~mm} \mathrm{NMR} \mathrm{tube}$ \\
\hline "day 1" \\
\hline
\end{tabular}

151.90

\subsection{7}

$\checkmark$

13.10

$\int$

766.74

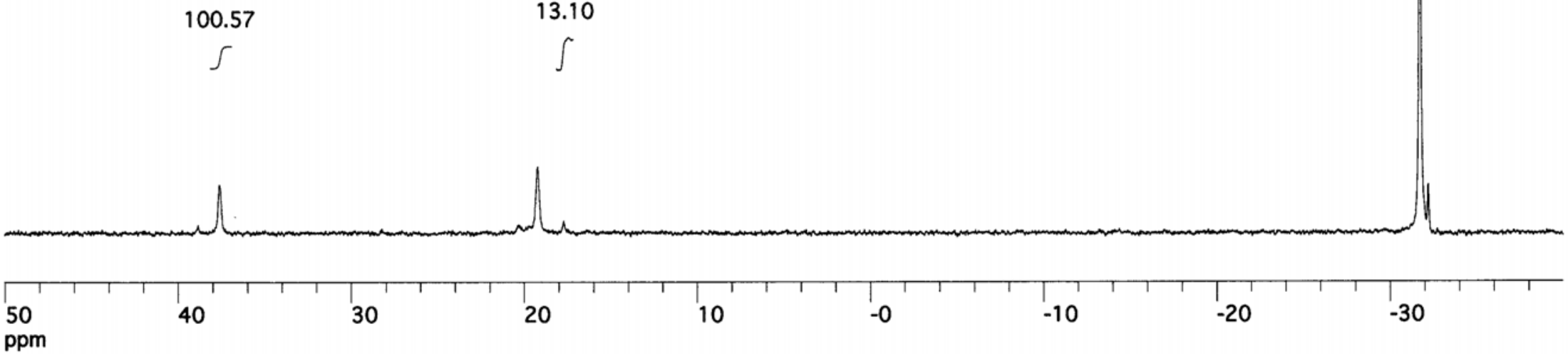

$\underset{\infty}{\infty}$ 


\begin{tabular}{|l|l|}
\hline $\mathrm{Pd}(\mathrm{OAc})_{2}$ & $0.017 \mathrm{M}$ \\
\hline $\mathrm{Na}_{2} \mathrm{CO}_{3}$ & $7.3 \mathrm{eq}$ \\
\hline TXPTS & $2.5 \mathrm{eq}$ \\
\hline $\mathrm{dA}$ & $2.0 \mathrm{eq}$ \\
\hline $1: 3: 2 \mathrm{D}_{2} \mathrm{O}-\mathrm{H}_{2} \mathrm{O}-\mathrm{CD}_{3} \mathrm{CN}$ \\
\hline $202.5 \mathrm{MHz}$ \\
\hline $10 \mathrm{~mm} \mathrm{NMR}$ tube \\
\hline "9 hours later" \\
\hline
\end{tabular}

488.12

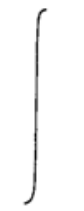

33.11

$\int$

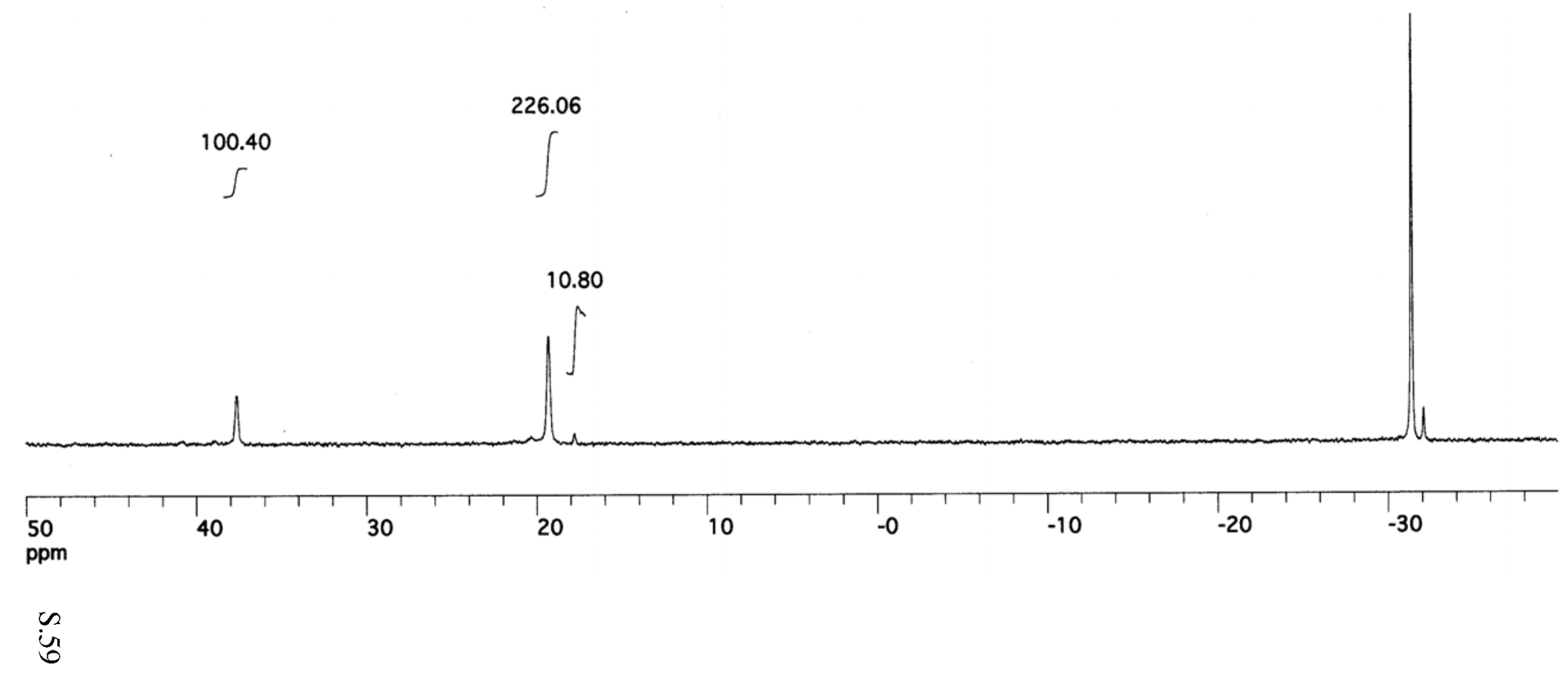




\begin{tabular}{|l|l|}
\hline $\mathrm{Pd}(\mathrm{OAc})_{2}$ & $0.017 \mathrm{M}$ \\
\hline $\mathrm{Na}_{2} \mathrm{CO}_{3}$ & $6.2 \mathrm{eq}$ \\
\hline TXPTS & $2.5 \mathrm{eq}$ \\
\hline $1 \mathrm{MedG}$ & $2.0 \mathrm{eq}$ \\
\hline $1: 3: 2 \mathrm{D}_{2} \mathrm{O}-\mathrm{H}_{2} \mathrm{O}-\mathrm{CD}_{3} \mathrm{CN}$ \\
\hline $202.5 \mathrm{MHz}$ \\
\hline $10 \mathrm{~mm} \mathrm{NMR} \mathrm{tube}$ \\
\hline "day 1" \\
\hline
\end{tabular}

331.78

555.34
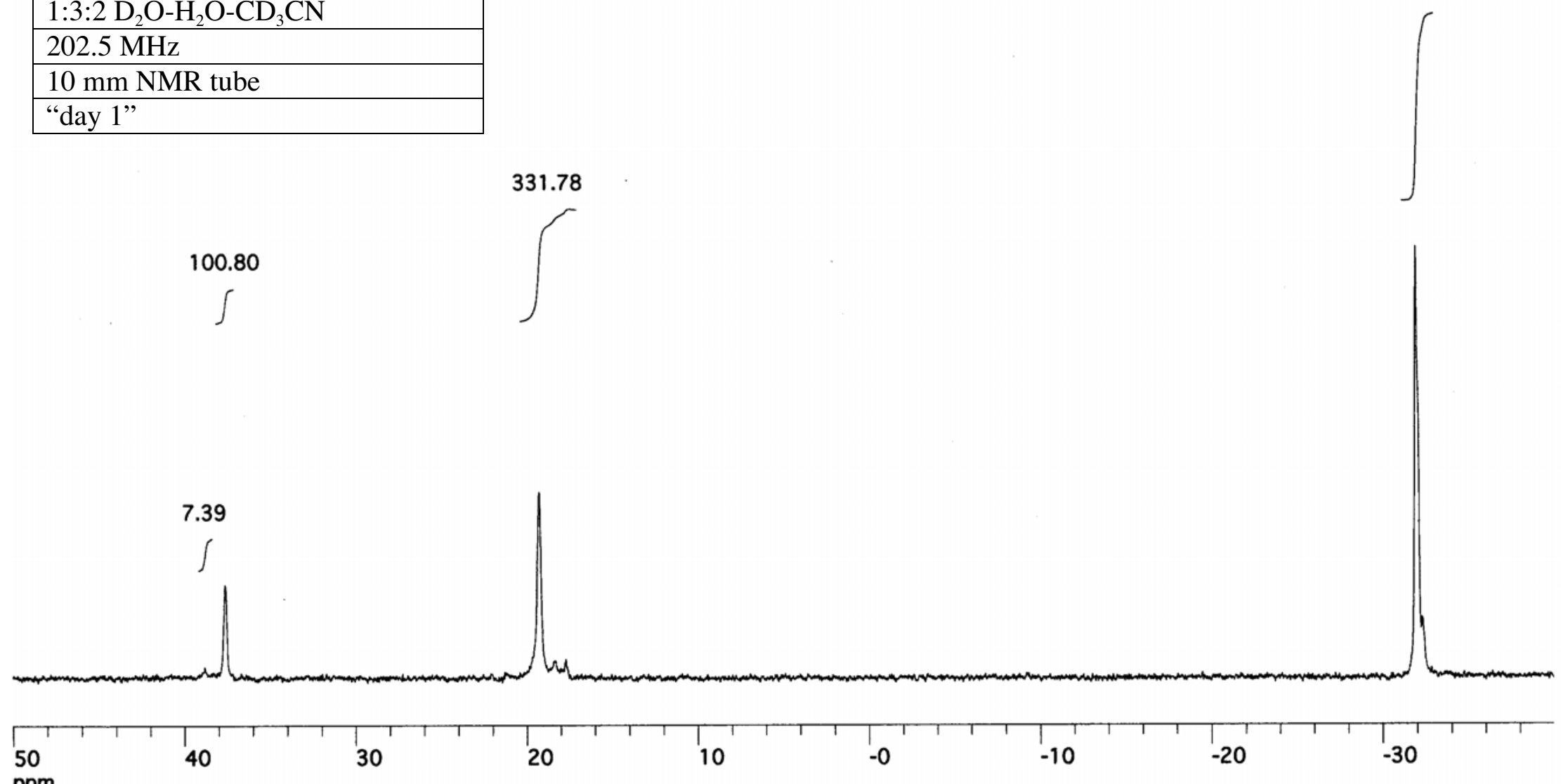

ppm

40

30

20

$-10$

$-20$

$-30$ 


\begin{tabular}{|l|l|}
\hline $\mathrm{Pd}(\mathrm{OAc})_{2}$ & $0.017 \mathrm{M}$ \\
\hline $\mathrm{Na}_{2} \mathrm{CO}_{3}$ & $6.2 \mathrm{eq}$ \\
\hline TXPTS & $2.5 \mathrm{eq}$ \\
\hline $1 \mathrm{MedG}$ & $2.0 \mathrm{eq}$ \\
\hline $1: 3: 2 \mathrm{D}_{2} \mathrm{O}-\mathrm{H}_{2} \mathrm{O}-\mathrm{CD}_{3} \mathrm{CN}$ \\
\hline $202.5 \mathrm{MHz}$ \\
\hline $10 \mathrm{~mm} \mathrm{NMR} \mathrm{tube}$ \\
\hline "day 2" \\
\hline
\end{tabular}

336.39

25.48

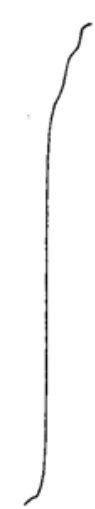

459.03

99.97
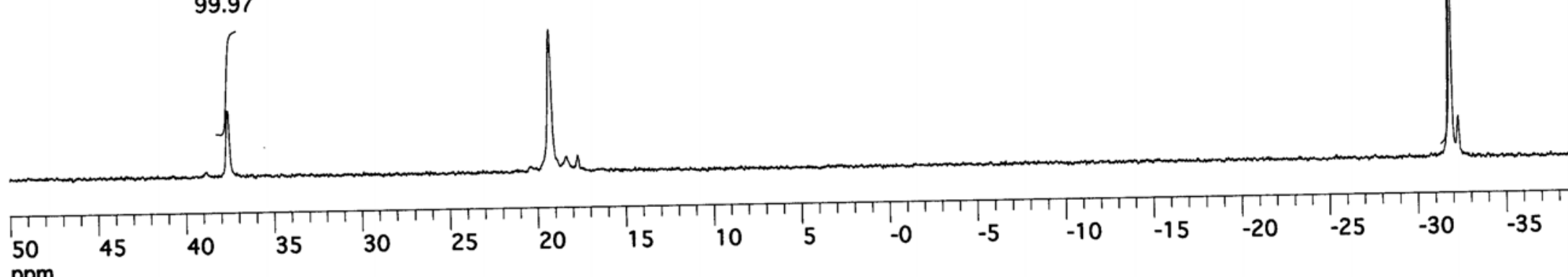

ppm

$\infty$ 


\begin{tabular}{|l|l|}
\hline $\mathrm{Pd}(\mathrm{OAc})_{2}$ & $0.017 \mathrm{M}$ \\
\hline $\mathrm{Na}_{2} \mathrm{CO}_{3}$ & $6.0 \mathrm{eq}$ \\
\hline $\mathrm{TXPTS}$ & $2.5 \mathrm{eq}$ \\
\hline $\mathrm{dG}$ & $2.0 \mathrm{eq}$ \\
\hline $1: 3: 2 \mathrm{D}_{2} \mathrm{O}-\mathrm{H}_{2} \mathrm{O}-\mathrm{CD}_{3} \mathrm{CN}$ \\
\hline $202.5 \mathrm{MHz}$ \\
\hline $10 \mathrm{~mm} \mathrm{NMR}$ tube \\
\hline "day 1"
\end{tabular}

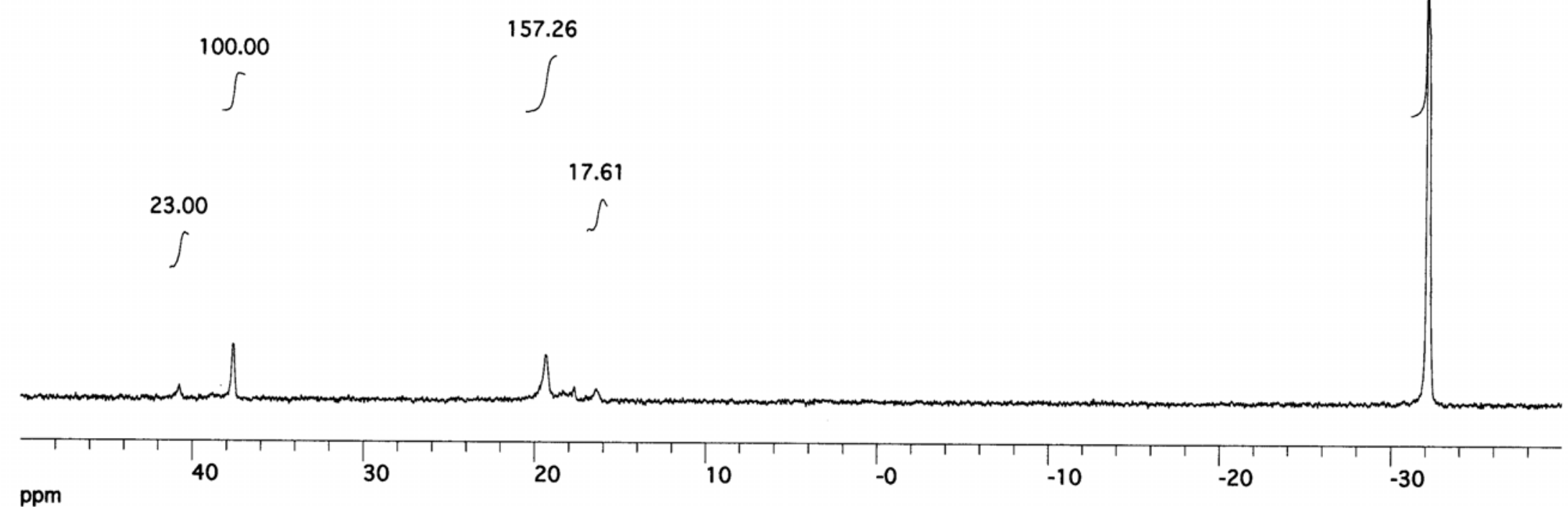




\begin{tabular}{|l|l|}
\hline $\mathrm{Pd}(\mathrm{OAc})_{2}$ & $0.017 \mathrm{M}$ \\
\hline $\mathrm{Na}_{2} \mathrm{CO}_{3}$ & $6.0 \mathrm{eq}$ \\
\hline $\mathrm{TXPTS}$ & $2.5 \mathrm{eq}$ \\
\hline $\mathrm{dG}$ & $2.0 \mathrm{eq}$ \\
\hline $1: 3: 2 \mathrm{D}_{2} \mathrm{O}-\mathrm{H}_{2} \mathrm{O}-\mathrm{CD}_{3} \mathrm{CN}$ \\
\hline $202.5 \mathrm{MHz}$ \\
\hline $10 \mathrm{~mm} \mathrm{NMR} \mathrm{tube}$ \\
\hline "day 2" \\
\hline
\end{tabular}

460.05

16.34

$\int$

$\int$

99.98

114.61

5

5

774.36

r

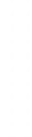




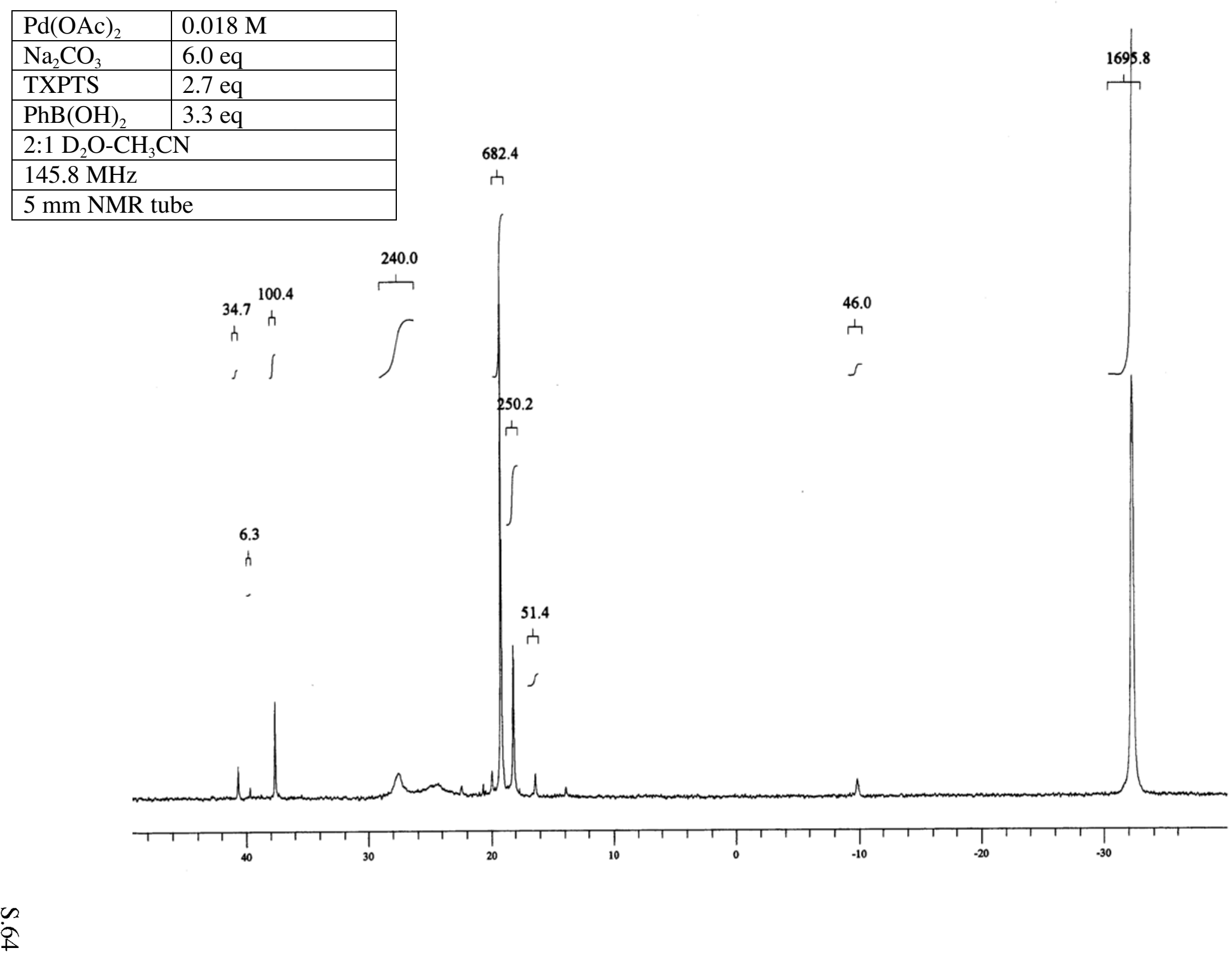




\begin{tabular}{|l|l|}
\hline $\mathrm{Pd}(\mathrm{OAc})_{2}$ & $0.018 \mathrm{M}$ \\
\hline $\mathrm{Na}_{2} \mathrm{CO}_{3}$ & $6.1 \mathrm{eq}$ \\
\hline $\mathrm{TXPTS}$ & $2.6 \mathrm{eq}$ \\
\hline $\mathrm{PhB}(\mathrm{OH})_{2}$ & $3.6 \mathrm{eq}$ \\
\hline $\mathrm{dA}$ & $2.0 \mathrm{eq}$ \\
\hline $2: 1 \mathrm{D}_{2} \mathrm{O}-\mathrm{CH}_{3} \mathrm{CN}$ \\
\hline $202.5 \mathrm{MHz}$ \\
\hline $5 \mathrm{~mm} \mathrm{NMR} \mathrm{tube}$ \\
\hline "day 1" \\
\hline
\end{tabular}
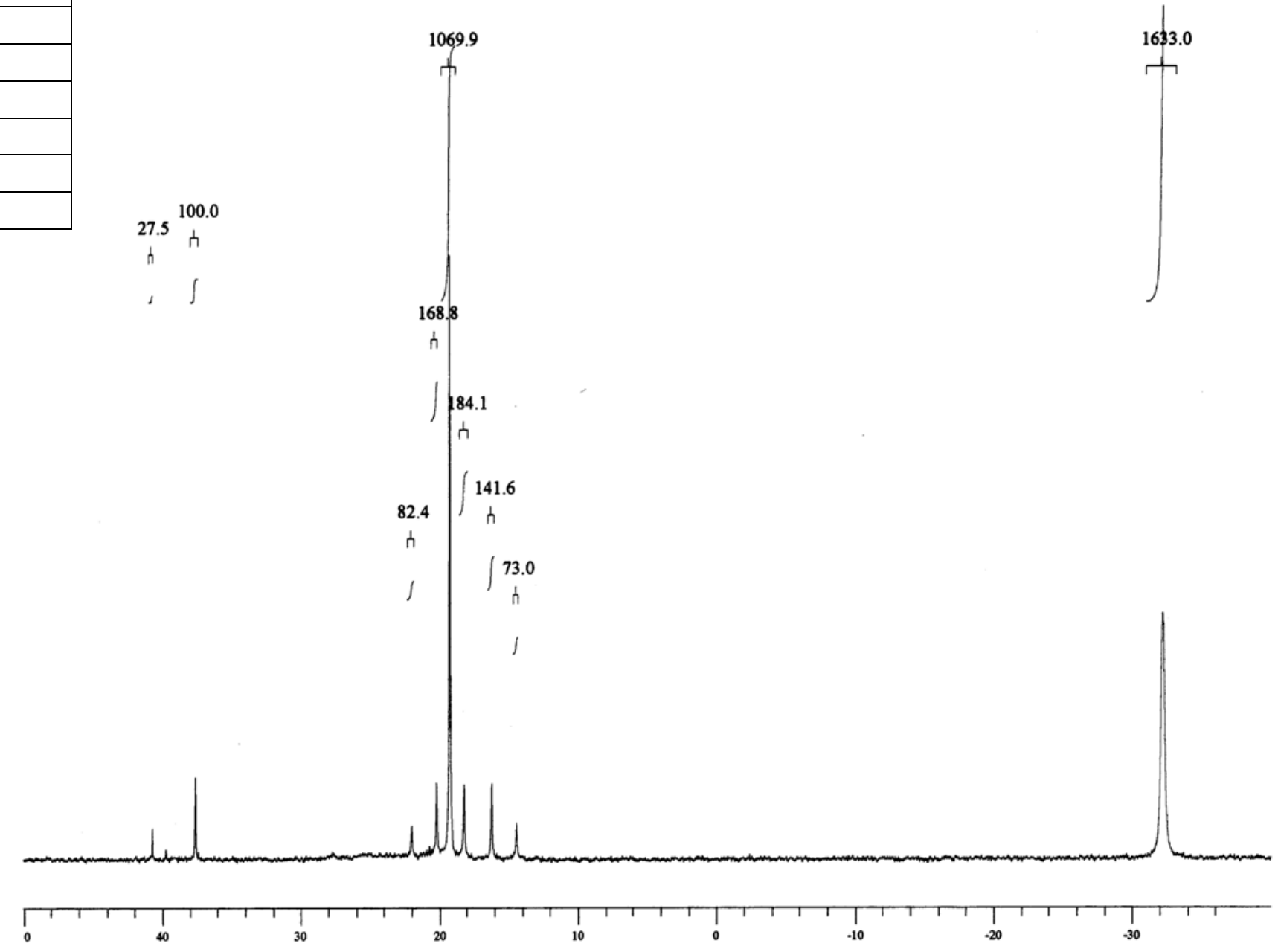


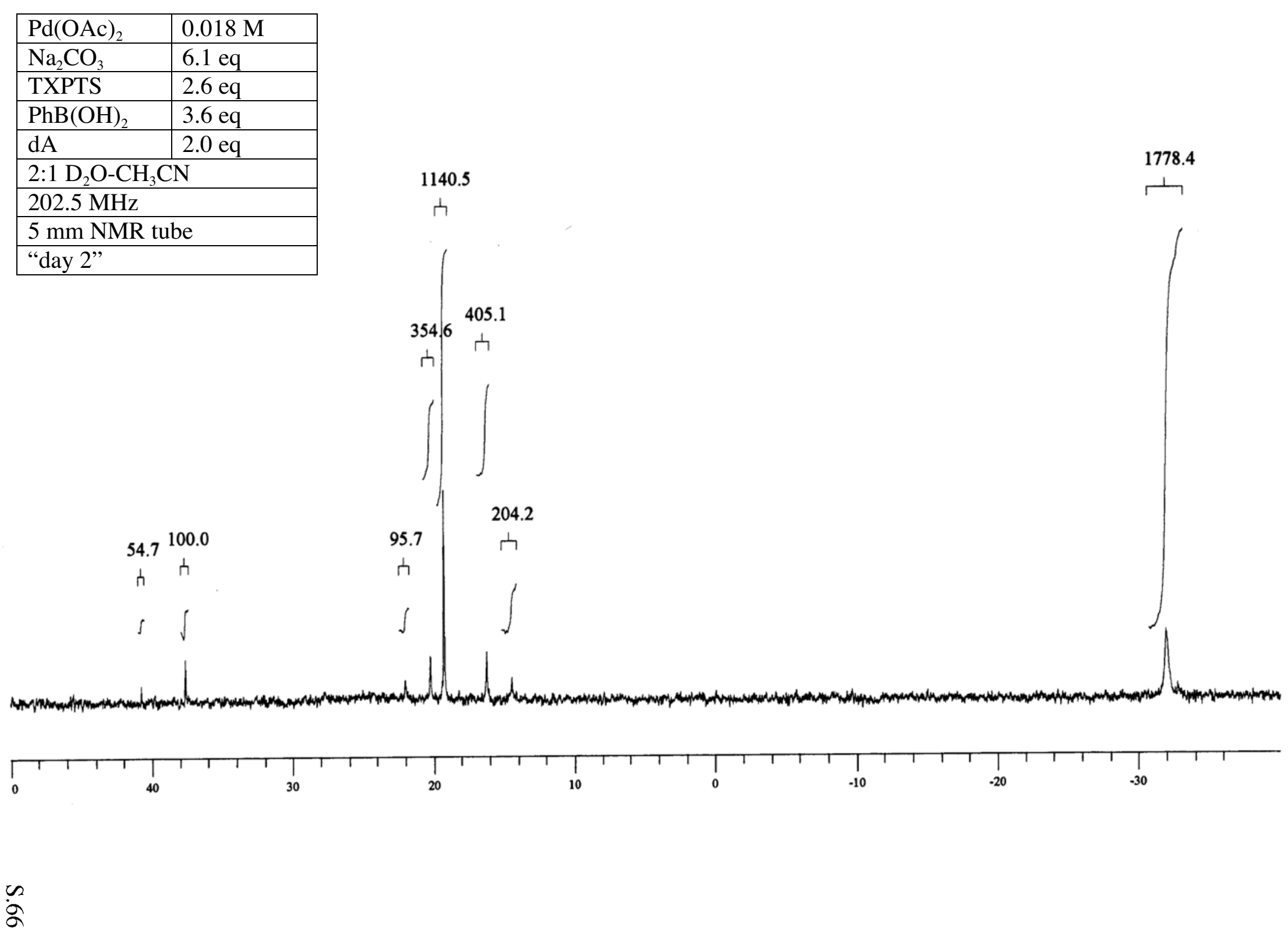




\begin{tabular}{|l|l|}
\hline $\mathrm{Pd}(\mathrm{OAc})_{2}$ & $0.018 \mathrm{M}$ \\
\hline $\mathrm{Na}_{2} \mathrm{CO}_{3}$ & $6.1 \mathrm{eq}$ \\
\hline TXPTS & $2.5 \mathrm{eq}$ \\
\hline $\mathrm{PhB}(\mathrm{OH})_{2}$ & $3.5 \mathrm{eq}$ \\
\hline $1 \mathrm{MedG}$ & $2.0 \mathrm{eq}$ \\
\hline $2: 1 \mathrm{D}_{2} \mathrm{O}-\mathrm{CH}_{3} \mathrm{CN}$ \\
\hline $202.5 \mathrm{MHz}$ \\
\hline $5 \mathrm{~mm} \mathrm{NMR} \mathrm{tube}$ \\
\hline "day 1" \\
\hline
\end{tabular}

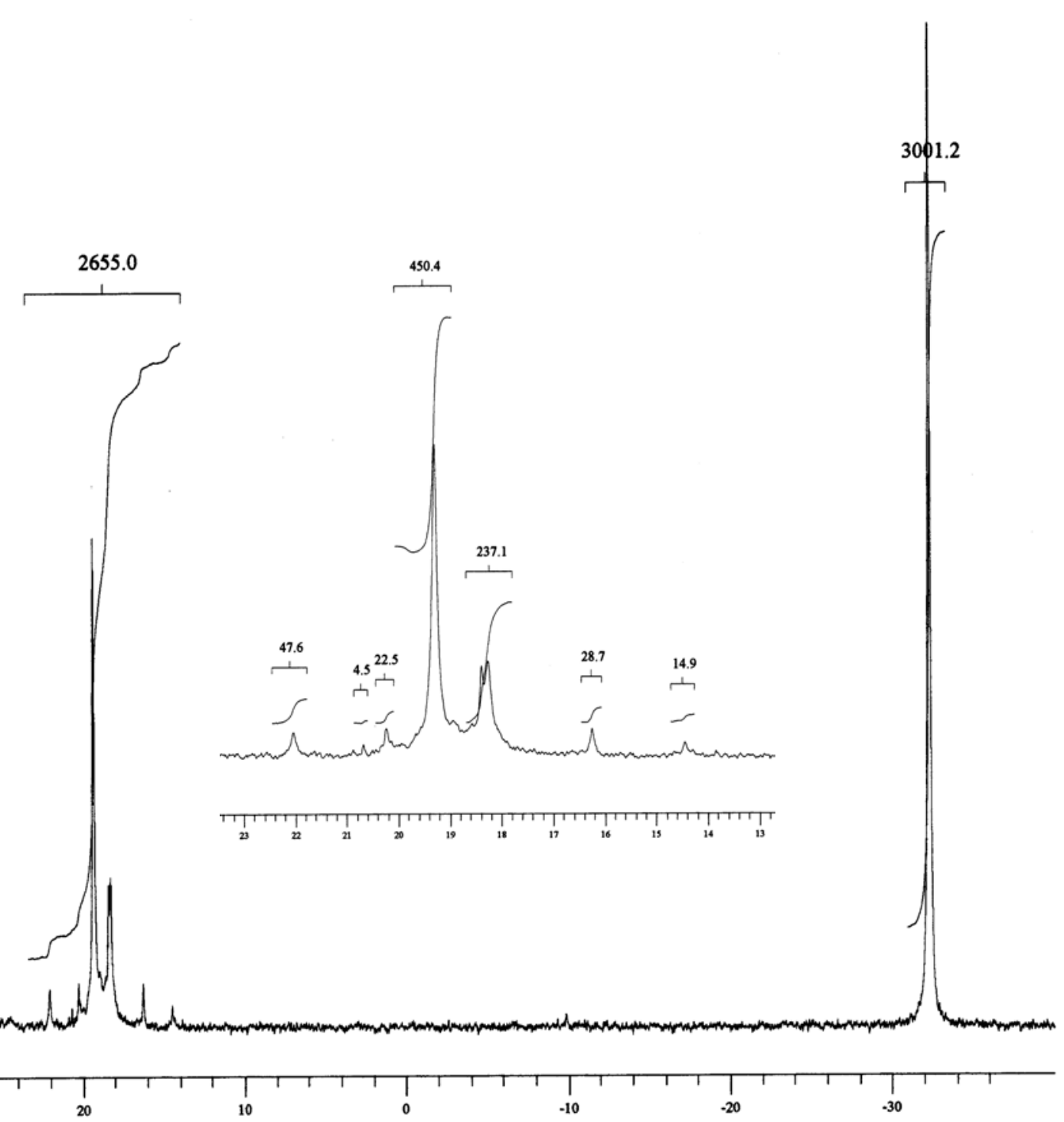

å 


\begin{tabular}{|l|l|}
\hline $\mathrm{Pd}(\mathrm{OAc})_{2}$ & $0.018 \mathrm{M}$ \\
\hline $\mathrm{Na}_{2} \mathrm{CO}_{3}$ & $6.1 \mathrm{eq}$ \\
\hline TXPTS & $2.5 \mathrm{eq}$ \\
\hline $\mathrm{PhB}(\mathrm{OH})_{2}$ & $3.5 \mathrm{eq}$ \\
\hline $1 \mathrm{MedG}$ & $2.0 \mathrm{eq}$ \\
\hline $2: 1 \mathrm{D}_{2} \mathrm{O}-\mathrm{CH}_{3} \mathrm{CN}$ \\
\hline $202.5 \mathrm{MHz}$ \\
\hline $5 \mathrm{~mm} \mathrm{NMR} \mathrm{tube}$ \\
\hline "day 2" \\
\hline
\end{tabular}

29.1 म

म

$\sim \int$
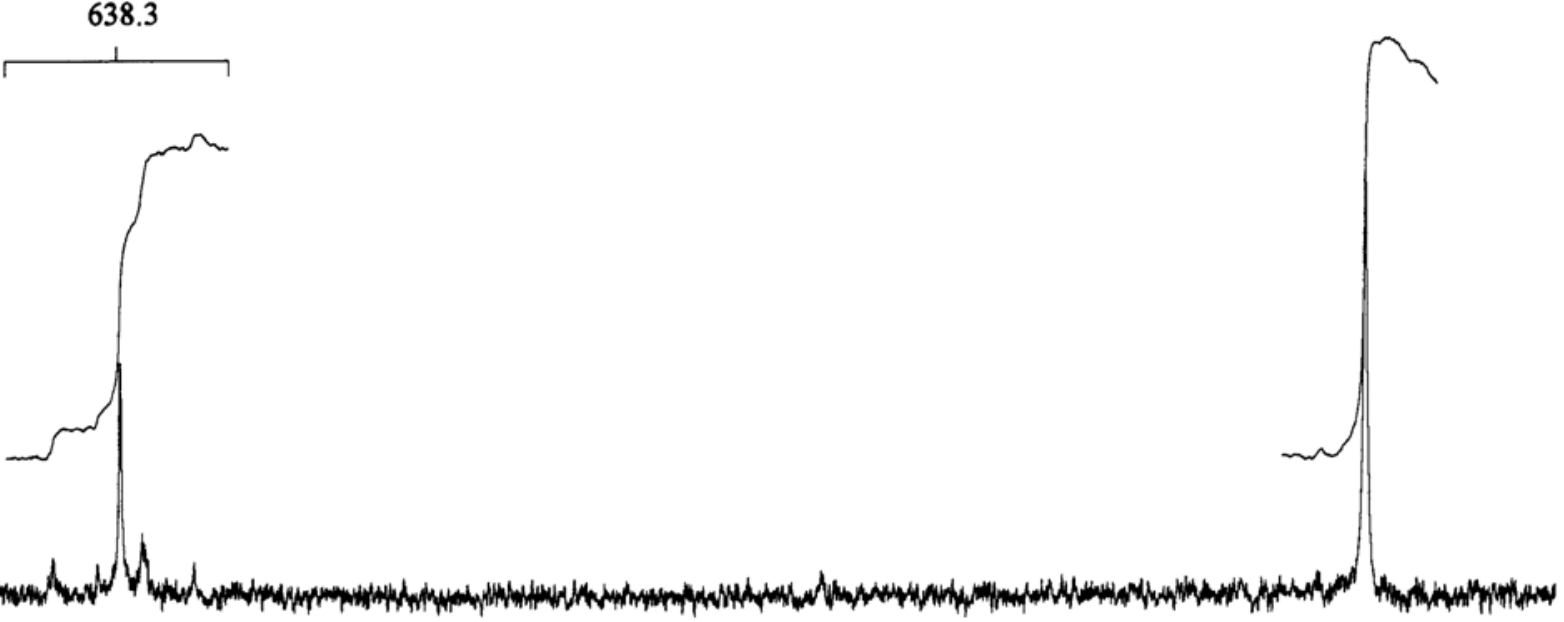

1.

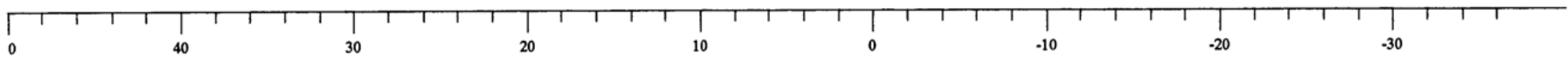

$\dot{\infty}$ 


\begin{tabular}{|l|l|}
\hline $\mathrm{Pd}(\mathrm{OAc})_{2}$ & $0.017 \mathrm{M}$ \\
\hline $\mathrm{Na}_{2} \mathrm{CO}_{3}$ & $6.1 \mathrm{eq}$ \\
\hline $\mathrm{TXPTS}$ & $2.5 \mathrm{eq}$ \\
\hline $\mathrm{PhB}(\mathrm{OH})_{2}$ & $3.4 \mathrm{eq}$ \\
\hline $\mathrm{dG}$ & $2.0 \mathrm{eq}$ \\
\hline $2: 1 \mathrm{D}_{2} \mathrm{O}-\mathrm{CH}_{3} \mathrm{CN}$ \\
\hline $202.5 \mathrm{MHz}$ \\
\hline $5 \mathrm{~mm} \mathrm{NMR}$ tube \\
\hline \multicolumn{2}{|l|}{ "day 1" } \\
\hline
\end{tabular}

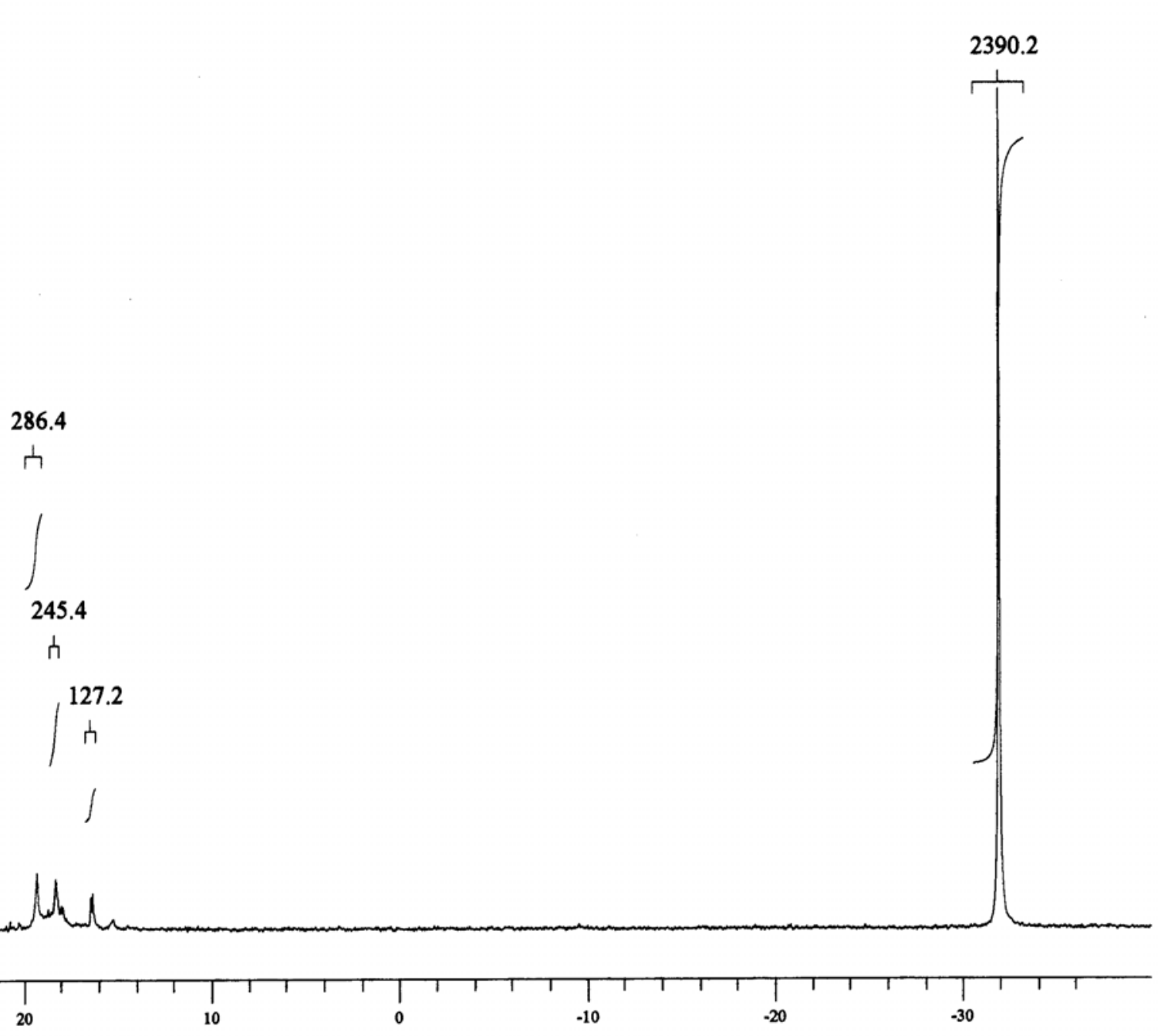




\begin{tabular}{|l|l|}
\hline $\mathrm{Pd}(\mathrm{OAc})_{2}$ & $0.017 \mathrm{M}$ \\
\hline $\mathrm{Na}_{2} \mathrm{CO}_{3}$ & $6.1 \mathrm{eq}$ \\
\hline $\mathrm{TXPTS}$ & $2.5 \mathrm{eq}$ \\
\hline $\mathrm{PhB}(\mathrm{OH})_{2}$ & $3.4 \mathrm{eq}$ \\
\hline $\mathrm{dG}$ & $2.0 \mathrm{eq}$ \\
\hline $2: 1 \mathrm{D}_{2} \mathrm{O}-\mathrm{CH}_{3} \mathrm{CN}$ \\
\hline $202.5 \mathrm{MHz}$ \\
\hline $5 \mathrm{~mm} \mathrm{NMR} \mathrm{tube}$ \\
\hline "day 2" \\
\hline
\end{tabular}

307.2

ウ

J

511.7

1

l
2918.6

$\longrightarrow$

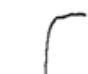

6.5100 .0

214.0

h $h$

$\pitchfork$

r

(1)

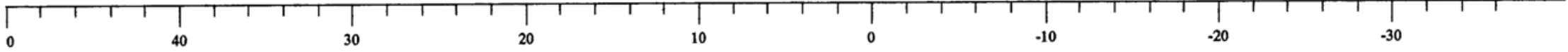

